\title{
THE QUERCUS FEEDING STIGMELLA SPECIES OF THE WEST PALAEARCTIC: NEW SPECIES, KEY AND
DISTRIBUTION (LEPIDOPTERA: NEPTICULIDAE)
}

Nieukerken, E. J. van \& R. Johansson, 2003. The Quercus feeding Stigmella species of the West Palaearctic: new species, key and distribution (Lepidoptera: Nepticulidae). - Tijdschrift voor Entomologie 146: 307-370, tables 1-3, figs. 1-179. [ISSN 0040-7496]. Published 1 December 2003.

The species of the Stigmella ruficapitella group occurring in the Western Palaearctic and feeding on Quercus are reviewed. We recognise 19 species, five of which are described as new: Stigmella fasciata sp. n. on Quercus pubescens from Slovenia, Croatia, Greece and Turkey, S. cocciferae sp. n. on Q. coccifera from Greece, Turkey and Israel, S. kasyi sp.n. from Afghanistan, possibly feeding on Quercus baloot, S. bicuspidata sp. n. from Turkey (host unknown) and $S$. karsholti sp. n. on Q. canariensis from Tunisia. Stigmella ilicifoliella (Mendes, 1918) comb. n., stat. rev. is removed from synonymy with S. suberivora (Stainton, 1869), it occurs in France, Spain and Portugal, partly sympatric with S. suberivora on Quercus rotundifolia, $Q$. ilex and $Q$. suber. Stigmella nigra Dufrane, 1955 is synonymised with S. ilicifoliella. S. suberivora, S. ilicifoliella, S. szoecsiella (Borkowski, 1972), S. macrolepidella (Klimesch, 1978), S. zangherii (Klimesch, 1951), S. dorsiguttella (Johansson, 1971), S. trojana Z. \& A. Laštuivka, 1998 and S. eberhardi (Johansson, 1971) are redescribed. A new diagnostic character on the forewing underside is described for male S. svenssoni (Johansson, 1971). Data on distribution and biology are given for all species, keys are given for males and for male and female genitalia. All known hostplants are listed, including several new records. The fauna on evergreen oaks, counting three species, is poor compared to that on deciduous oaks and compared to the genus $E_{c}$ toedemia. The Eastern Mediterranean region, with about five endemic species, has a more diverse fauna than the Western part, were only three endemic species occur.

Correspondence: Erik J. van Nieukerken, National Museum of Natural History, Naturalis, PO Box 9517, 2300 RA Leiden, The Netherlands. E-mail: nieukerken@naturalis.nl

Keywords. - Taxonomy; new species; hostplant; leafmine; Stigmella ruficapitella group; Fagaceae; Quercus; Castanea, keys.

Oaks (Quercus species) harbour an enormous diversity of herbivorous insects, and in Europe there are more insects on oaks than on any other tree (Southwood 1961). Leafminers are among the commonest insects on oaks: Hering (1957) recognised about 200 species in Europe, the majority being Lepidoptera. Identification of these leafminers has often been difficult and many taxonomic changes have occurred since Hering's work, making identification often almost impossible.

Within the Nepticulidae, the approximately 26 leafmining species of Ectoedemia Busck, 1907 (s. str.) and the few barkmining species in Ectoedemia (Zimmermannia) Hering, 1940 feeding on Quercus have been revised previously (Van Nieukerken 1985, see Laštüvka \& Laštủvka 1998 for one or two additional species). The species of Stigmella, all belonging to the Stigmella ruficapitella group in the sense of Johansson \& Nielsen (1990), have always given identification problems, until the junior author provided their first critical revision (Johansson 1971). All the northern European species, including the four non Quercusfeeders in this group, were completely treated by Johansson $\&$ Nielsen, including colour illustrations of adults.

In material from the Mediterranean region and Middle East we found five hitherto undescribed species, either reared from Quercus, or believed to be Quercus miner. Also, since Johansson (1971), many new data on the other species of the ruficapitella group have become available, and some new species had been described, especially from the Mediterranean region. In this paper we therefore describe the new species, in- 

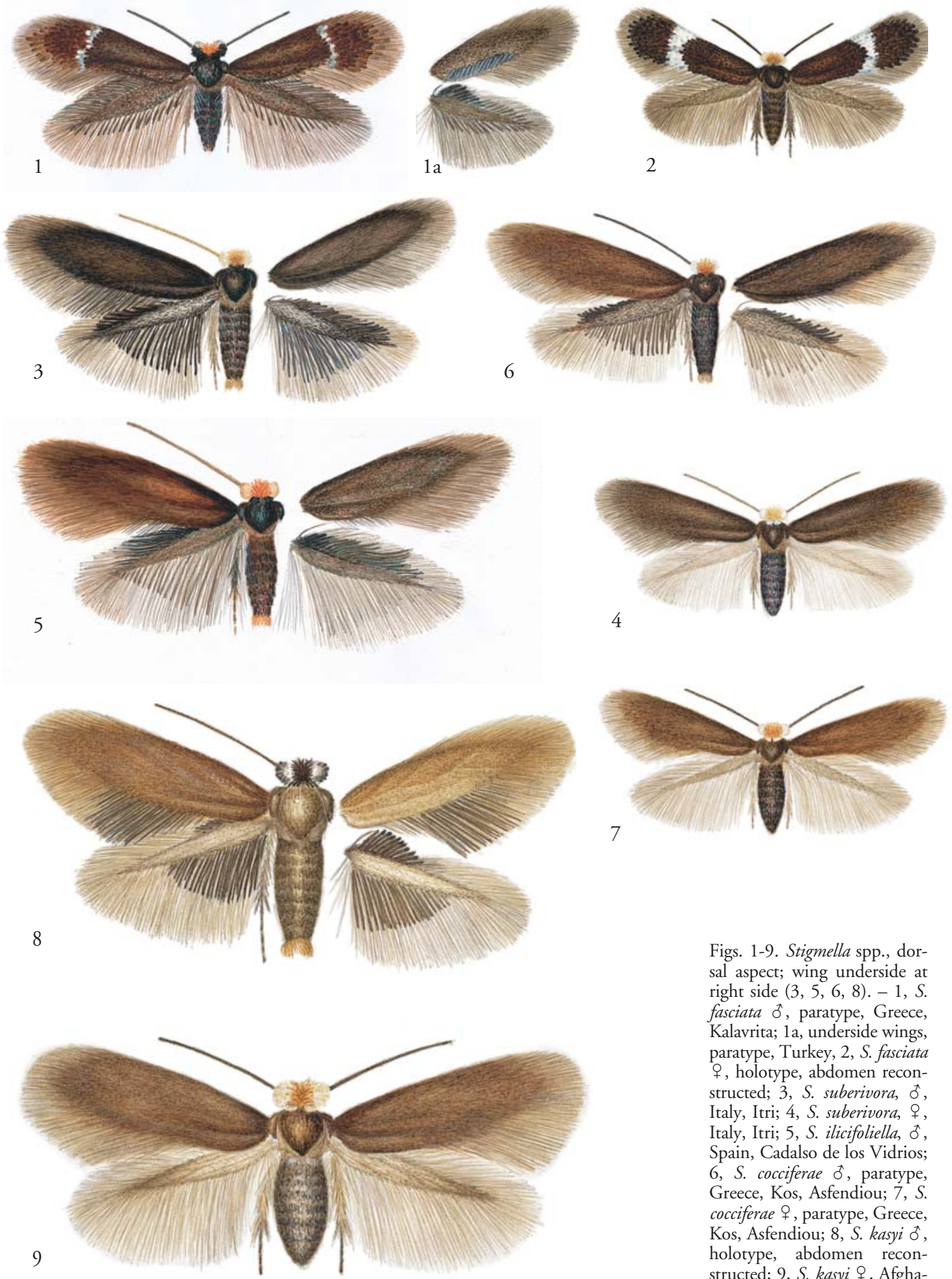

Figs. 1-9. Stigmella spp., dorsal aspect; wing underside at right side $(3,5,6,8) .-1, S$. fasciata $\hat{\sigma}$, paratype, Greece, Kalavrita; 1a, underside wings, paratype, Turkey, 2, S. fasciata $\$$, holotype, abdomen reconstructed; 3, S. suberivora, ô, Italy, Itri; 4, S. suberivora, o, Italy, Itri; 5, S. ilicifoliella, ô, Spain, Cadalso de los Vidrios; 6, S. cocciferae ô, paratype, Greece, Kos, Asfendiou; 7, S. cocciferae 9 , paratype, Greece, Kos, Asfendiou; 8, S. kasyi ô, holotype, abdomen reconstructed; 9, S. kasyi 우, Afghanistan, abdomen reconstructed. All on same scale, ca $11 \times$. 
clude keys to all western Palaearctic Quercus (and Castanea) mining Stigmella species and give additional data on the other oak mining species. Species not treated by Johansson \& Nielsen (1990) are redescribed. With the above mentioned works on Ectoedemia, it is now possible to identify all western Palaearctic Nepticulidae, feeding on Quercus and Castanea.

\section{MATERIAL AND METHODS}

\section{Material}

We studied material from a wide range of sources, museums, private collections and material collected by ourselves. For the collections we use the abbreviations from Samuelson et al. (2001) with the following additions:

AL Collection A. Laštu̇vka (Prostejov, Czech Republic)

AV Collection A. Vives-Moreno (Madrid, Spain)

BÅB Collection B.Å. Bengtsson (Färjestäden, Sweden)

CG Collection C. Gielis (Lexmond, Netherlands)

CVDB Collection C. van den Berg (Hoogland, Netherlands)

GB Collection G. Baldizzone (Asti, Italy)

GD Collection G. Derra (Reckendorf, Germany)

HS Collection H. Steuer (Bad Blankenburg, Germany)

HW Collection H. W. van der Wolf (Nuenen, Netherlands)

JJ Collection J. Junnilainen (Vantaa, Finland)

JCK Collection J.C. Koster (Callantsoog, Netherlands)

JHK Collection J. H. Kuchlein (Wageningen, Netherlands)

JL Collection J. A.W. Lucas (Rotterdam, Netherlands)

MC Collection M. F. V. Corley (Faringdon, United Kingdom)

MHNMMuseum d'Histoire Naturelle de Marseille (France) (containing collection of the late R. Buvat)

PT Collection P. Triberti (Verona, Italy)

RJ Collection R. Johansson (Växjö, Sweden), to be deposited in ZMUC

RS Collection R. Sutter (Bitterfeld, Germany)

VZ Collection V. Zolotuhin (Uljanovsk, Russia)

WB Collection W. Biesenbaum (Velbert-Langenberg, Germany)

We provide only detailed material lists for the new species and the rare species, the many records of the commoner species, especially in northern Europe are summarised, except for the faunistical interesting records, which are cited in full. A complete listing of material and literature records, including coordinates, is available as an Excel file upon request from the se- nior author or from his website (http://www.naturalis.nl/nieukerken). Material lists are arranged alphabetically by country and locality; when applicable, the province name is given as first item in the locality. Depositories are only given after the last record in a row, when more than one record refer to the same collection. Unpublished records we received from colleagues are listed under 'Additional records' when they are of faunistic interest, otherwise they are only used for the maps.

\section{Maps}

We provide distribution maps, prepared with DMAP 7.0 (Morton 2000). For these maps we have used the examined material supplemented with records from previous revisions (Borkowski 1972, Johansson 1971), the data from the original descriptions, databases provided by B. Gustafsson (Sweden), L. Aarvik (Norway, including all Norwegian records) and J. Buszko (Poland), records provided by M. F. V. Corley, A. and Z. Laštuivka and S. Whitebread and those post 1971 publications that we could find with reliable records based on adults. Also the few publications accompanied by genitalia figures prior to 1971 (here cited under the correct species) have been used. The references to these are included in the above mentioned Excel file. For S. basiguttella we also accepted records on the basis of mines and older records, and the British records of the common species may include some records which were based on leafmines; for the more critical species we have excluded all records that were clearly based on mines alone. UTM coordinates or longitude/latitude when not given on labels or in references, were mainly derived from a few major internet gazetteers and one atlas (IGN 1997-2003, NIMA 2003, Ordnance-Survey 2003, Times 2000) and further supplemented by a number of sources, including the map collection of EvN. Most maps show the approximate distribution of (some) hostplants as shaded area, see below.

\section{Preparation and illustrations}

Genitalia preparations were embedded in Euparal or Canada balsam, following the methods described in Van Nieukerken et al. (1990). Photographs of genitalia were taken with a Zeiss AxioCam digital camera attached to a Zeiss Axioskop H, using Carl Zeiss AxioVision 3.0.6 software. Drawings of genitalia were prepared by RJ with a simple microscope and updated with the help of photographs taken by a Zeiss Axioskop H. Watercolours of adults were made by RJ using diffuse natural light; in some specimens abdomens were reconstructed, in figures 27 and 34 the left wings were not drawn, but digitally mirrored. Several watercolours and genitalia figures used here were published previously by Johansson \& Nielsen (1990). 


\section{Descriptions}

Species fully described by Johansson \& Nielsen (1990) are not redescribed, except $S$. suberivora, but diagnostic characters are provided and new information is given when applicable. All other species are described in full. Measurements are given in tables 2-4, when the number of observations is four or higher, we provide also mean and standard deviation. Measurements of genitalia were recently obtained from digital images, using AxioVision, $20 \times$ objective for male genitalia and usually $10 \times$ for females. Some older measurements were taken with an eyepiece graticule, with a precision of about $5 \mu \mathrm{m}$. Capsule length was measured from middle of vinculum to middle of uncus, excluding any projections; valva length from tip of posterior process to ventral edge, not the sublateral process; aedeagus length was measured from the sclerotized tube, excluding any protruding vesica parts. Bursa length is measured from cloaca to anterior tip. Forewing length was measured from tip of cilia to attachment on thorax, usually at magnification of $20 \times$. Antennal segment counts include scape and pedicel. See also some observations below.

All synonyms are given, references only to original descriptions and most relevant references, most of them post-1971. Morphological terms follow Van Nieukerken et al. (1990).

\section{Hostplants}

Nomenclature of Fagaceae follows Govaerts \& Frodin (1998), deviating names in recent European treatments (Amaral Franco 1990, Schwarz 1993) are given in the hostplant list (table 1). Hostplant records of reared material, seen by us, have been provided with two asterisks $\left({ }^{* *}\right)$; records of material seen by us, based either on possible mines or by inference on the basis of collected adults in pure stands of a single oak species, have been provided with a single asterisk $\left(^{*}\right)$. All other records are provided with a reference. Literature records prior to 1971 are neglected, except for $S$. basiguttella, or those for which the identity has been confirmed later by material. Records of unidentified Stigmella mines on oak species for which we have no other records, are given in the hostplant list. The distribution maps of Quercus are reconstructed from maps in Jalas \& Suominen (1976), Menitskij (1984) and Rikli (1942-1943).

\section{Remarks on structure and identification}

\section{Externals}

Adult males are usually characteristic and can often be identified on externals alone. Some characters may vary, and in doubtful cases genitalia should always be examined, especially in areas where the fauna is less well known. The wing pattern varies from complete- ly and uniformly dark, usually with some shine and a somewhat purplish tip, to dark wings with a variation of pale pattern. To judge colour, specimens should be viewed under natural daylight, or in the case of microscope lights it is advisable to use a blue filter. The colour of older collection material has almost always faded into a paler tinge.

Most males show some kind of special or androconial scales, although they may be rather indistinct. Most conspicuous are the spatulate scales extending into the hindwing fringe of several of the species; these may be upcurved in prepared material and are then more difficult to measure. The length of these scales is an important character to separate $S$. atricapitella and ruficapitella.

All species except fasciata have distinct anal tufts, inserted on strongly sclerotized plates on segment 8 , which can best be observed in genitalia slides; sometimes these plates remain attached to the genitalia rather than the abdomen when the genitalia are severed from the abdomen. The colour of these tufts may also be diagnostic. The external genitalia (valves) are usually covered with scales of the same colour as the tufts.

Females can hardly be identified on externals, with the exception of $S$. fasciata. Stigmella macrolepidella and $S$. roborella can be recognized by a rather pointed ovipositor. Red-headed species can more or less be grouped by the number of antennal segments: the evergreen oak feeders have more segments than the deciduous oak feeders (table 2).

\section{Genitalia}

The value of genitalia characters is obvious, but also in genitalia variation can occur. In the male genitalia we observed especially variability in length of the vinculum (e. g. in S. roborella) and shape of the valva. In the females the shape and size of the accessory sac can vary, and this is probably partly an effect of maturation: virgin females probably have the sac still collapsed but after mating it extends fully and spines can be more spread out. Examples of these are shown here in S. karsholti and S. samiatella. In addition the shape can be influenced by the preparation technique: the very thin structures of the bursa can then also collapse.

In general specimens from South Europe of widespread species are often smaller than specimens from North Europe: this also affects the genitalia and can lead to a smaller numbers of spines in the accessory sac.

\section{Measurements}

Microlepidopterists rarely use measurements as diagnostic characters, although we find them helpful in some cases. Counting the number of antennal segments can be useful as a rough check for recognizing species, also to separate them from species in other groups (see above). Here S. suberivora and S. ilicifoliel- 
la show a significant difference in the number of segments, especially when the ratio with forewing length is taken. Although wingspan or alar expanse is a frequently used measure, it cannot be measured as exactly as forewing length, because it depends on the way of mounting; it is only used here as a rough indication. Forewing length is defined as the length from the wing base at costa to the tip of the fringe (see table 2).

Measurements of genitalia can be rather characteristic. The error in measurements is, depending on magnification, in the order of $5 \mu \mathrm{m}$. Measurements are usually taken from mounted genitalia; because the viewing angle depends on the way of mounting (aedeagus included in capsule or not, amount of squashing), there is an extra amount of uncertainty. It is also not always easy to decide where to start the measurement (i.e. tip of aedeagus). In really squashed slides we did not measure the widths, because the genitalia tend to spread in lateral direction. The lengths remain relatively intact.

We have hardly measured female genitalia, except in the case of separating the similar species of the suberivora group. It is often difficult to determine markers from where to measure, and the tender structures often collapse more or less during preparation.

\section{Mines, larvae and hostplant}

The species described here make relatively similar leafmines. Characters of leafmines (egg-position, length, width of frass) depend on habits of the female, the larva, but also on leaf thickness, host species and external factors such as weather conditions. This makes many characters variable and identification on the characters of the mine alone often unreliable. The only species with very distinctive mine forms are Stigmella fasciata and S. basiguttella; records of these species can usually be accepted on the basis of mines alone. Still it is clear that the other species also tend to make different mines, and with experience it is sometimes possible to predict more or less what species will emerge. However, since existing descriptions show contradicting statements, e. g. regarding the egg position of S. samiatella, and since a thorough statistical analysis of the characters of mines of which the identity is established with $100 \%$ certainty (by rearing or larval characters) is still lacking, we find it unwise to give records on the basis of vacated mines only. In some countries, particularly the British Isles, the Netherlands and Poland, tradition has been to identify oak mines on the basis of vacated mines alone and single characters such as egg position. Although this may be correct in a fair proportion of cases, we think that many of such records, particularly of rarer species, need to be viewed with care. The leading British specialist, the late A. M. Emmet, was at first also very critical to identifications based on mines (Emmet 1976a), but later (Emmet 1985) he wrote: 'I have written that the atricapitella group cannot be identified reliably on the evidence of mines alone: it is now possible to do so in many instances'. He may have been right, and undoubtedly had a lot of field experience in the British Isles. However, the characters he used are not well circumscribed and probably not always applicable to other geographical areas. We have therefore excluded records of the rarer species in Britain based on mines alone from the maps. We would suggest that authors illustrating leafmines (e.g. on the internet!) indicate whether the identification was based on reared adult, larval characters or mine form only. If reared, then an illustration of the adult and its genitalia would be helpful.

Hostplant identity cannot be used as an identification character for the species on deciduous oaks or chestnut, because all species of Quercus and Castanea appear to have a variable fauna and no strictly monophagous Stigmella are known on these hosts. Still, it seems that most records on Castanea are indeed correctly identified as S. samiatella; previous records of S. ruficapitella or S. atricapitella have either been proven wrong or are doubtful, because they were based on mines alone. The only other species recorded with certainty from Castanea is S. basiguttella. Larval characters may be diagnostic, but are only described for most northern species, except $S$. samiatella (Gustafsson \& Van Nieukerken 1990).

\section{TAXONOMY}

\section{Stigmella ruficapitella group}

Nepticula ruficapitella group: Johansson 1971: 241.

Nepticula hemargyrella group: Johansson 1971: 241.

Nepticula atricapitella group: Emmet 1976a: 239.

Stigmella ruficapitella group: Puplesis 1984: 583, Puplesis 1985: 150, Kemperman et al. 1985: 40, Van Nieukerken 1986b: 13, Johansson \& Nielsen 1990: 224, Puplesis 1994: 155; Van Nieukerken \& Liu 2000: 151.

Stigmella hemargyrella group: Van Nieukerken 1986b: 13; Puplesis 1994: 150.

Stigmella suberivora group: Kemperman et al. 1985: 47.

Stigmella castanopsiella group: Puplesis 1984: 583, Puplesis 1985: 150, Puplesis 1994: 164.

\section{Diagnosis}

There are no fool-proof characters for recognizing members of this group, but uniformly coloured males, with strong anal tufts inserted on well sclerotized plates belong here. In Europe the only species which may be confused with these are: S. paradoxa (Frey, 1858), S. inopinata A. \& Z. Laštuivka, 1990, both in the $S$. paradoxa group and S. lonicerarum (Frey, 1857) within the $S$. ruficapitella group. The species of the $S$. thuringiaca group also have similar tufts, but these species are much smaller, and usually more greyish, with less shine. Also the rather similar S. tristis 

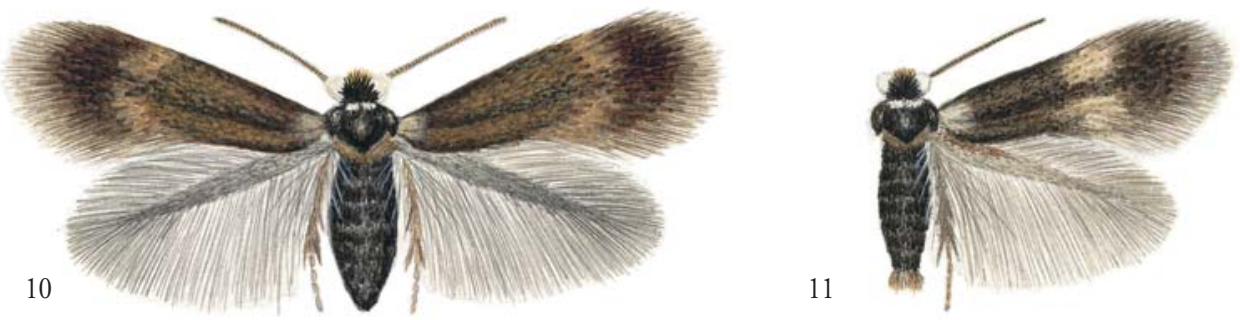

13
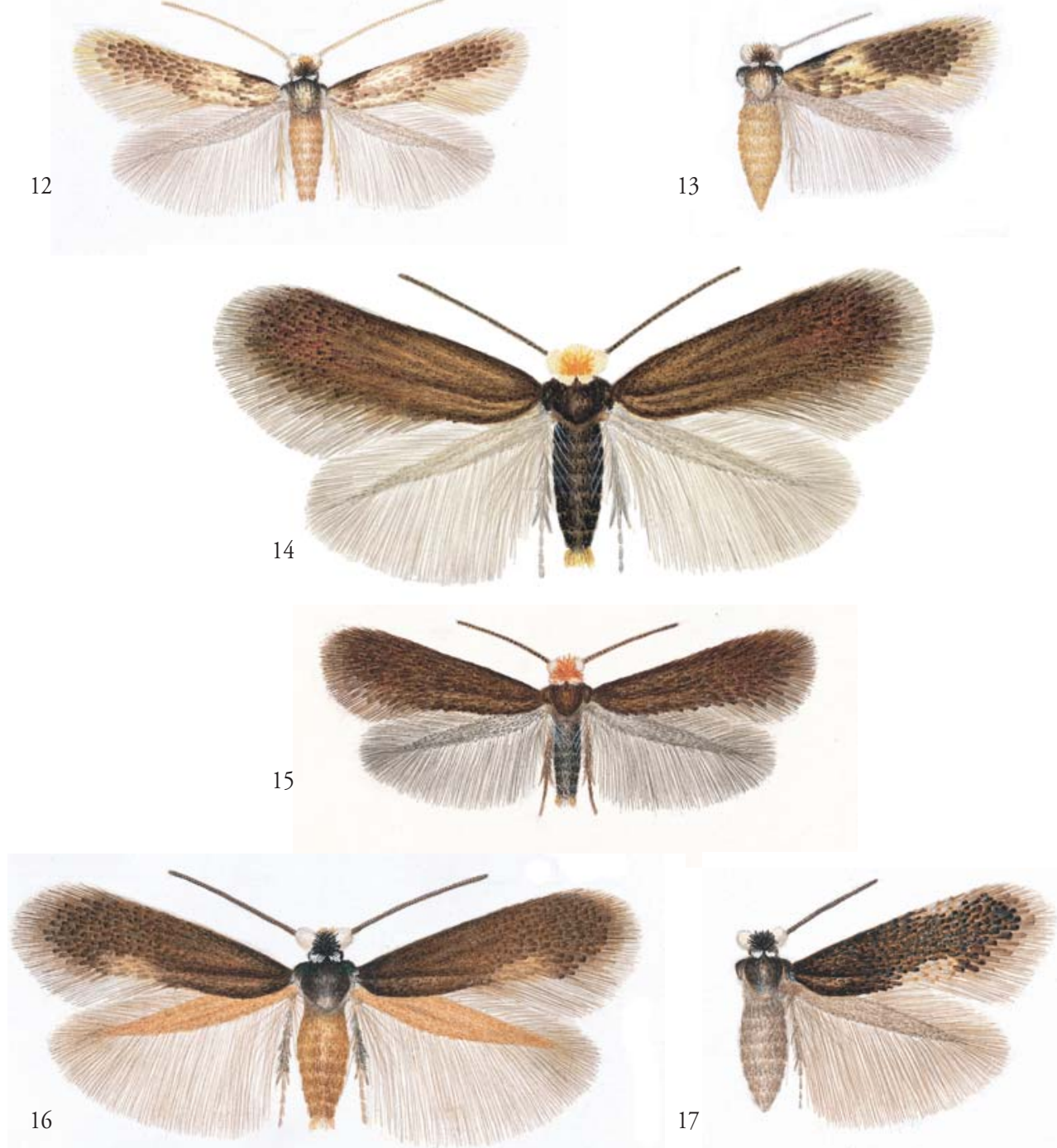

Figs. 10-17. Stigmella spp., dorsal aspect. - 10, S. basiguttella ㅇ, lectotype; 11, S. basiguttella ô, Germany, Lüneburger Hei-

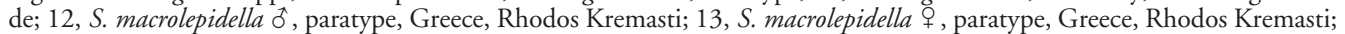
14, S. svenssoni ơ, Sweden, Högsby; 15, S. szoecsiella, ô, Italy, Vasto, abdomen reconstructed; 16, S. zangherii, ô, Hungary, Törökbálint; 17, S. zangherii, o , Hungary, Törökbálint; All on same scale, ca 13×. 


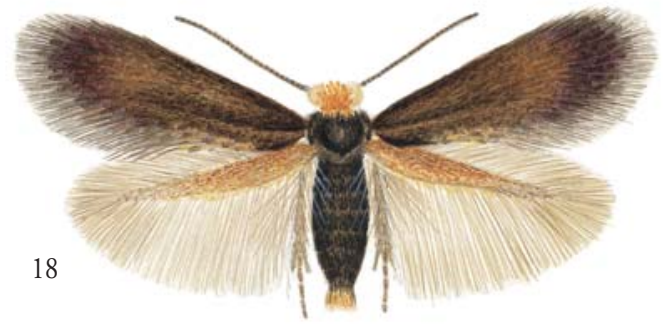

19
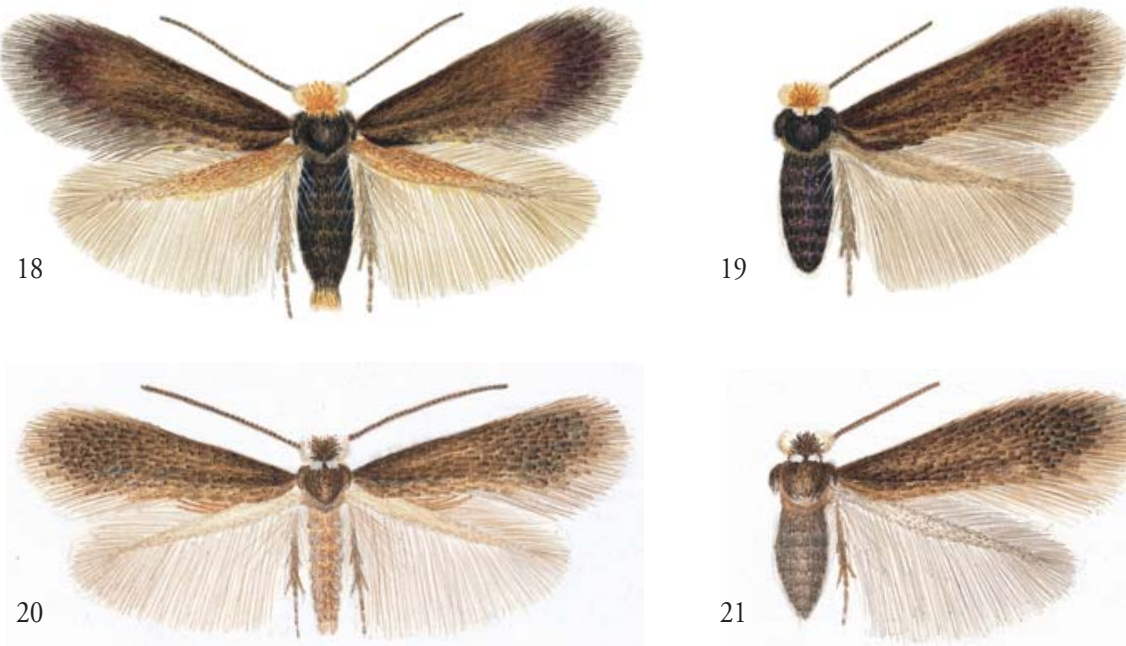

21
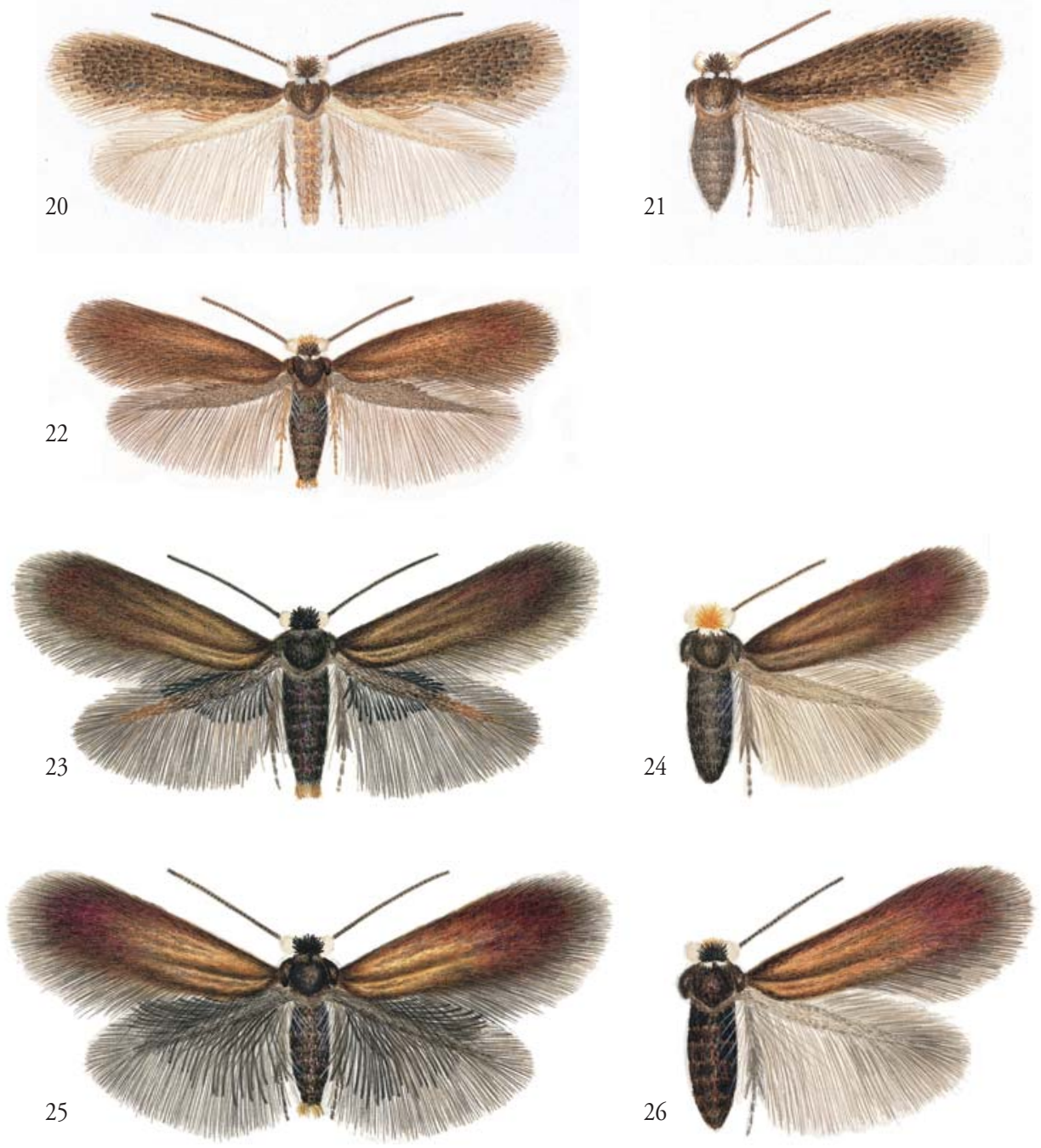

Figs. 18-26. Stigmella spp., dorsal aspect. - 18, S. dorsiguttella ô, Sweden, Högsby, paratype; 19, S. dorsiguttella + , Sweden, Högsby, holotype; 20, S. trojana ơ, paratype, Greece, Thesprotika; 21, S. trojana 9 , paratype, Greece, Kozani; 22, S. bicus-

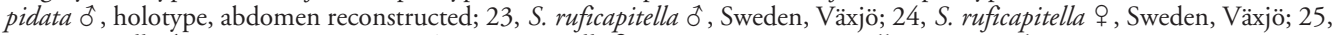
S. atricapitella ơ, Germany, Bremen; 26, S. atricapitella $\$$, Germany, Bremen. All on same scale, ca $13 \times$. 
Table 1. List of hostplants of Western Palaearctic species of the Stigmella ruficapitella group feeding on Fagaceae. Only certain records are provided, sources are given under the respective species. The hosts and the Stigmella species are given in alphabetic order, synonyms of hosts in recent European literature are included.

Hostplant

Stigmella species

Castanea Miller

C. sativa Miller

Quercus L.

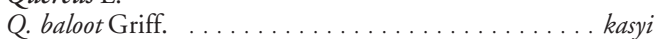

Q. canariensis Willd. ......................... karsholti

(= Q. mirbeckii Durieu)

Q. castaneifolia C. A. Mey . . . . . . . . . . . . basiguttella

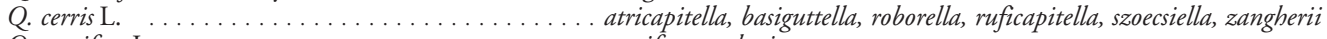

Q. coccifera $\mathrm{L} . \ldots \ldots \ldots \ldots \ldots \ldots \ldots \ldots \ldots \ldots \ldots \ldots \ldots \ldots$ cociferae, suberivora

Q. faginea Lam. ..................... Stigmella sp. [vacated mines and larvae in Tunisia, Spain, EvN]

Q. frainetto Ten. . . . . . . . . . . . . . . basiguttella, Stigmella sp. (as ruficapitella and samiatella) (Buhr 1940,

Q. ilex L.

Draghia 1975, Popescu-Gorj \& Draghia 1966)

(= Q. ilex ssp. ilex)

Q. ithaburensis Decne ssp. ithaburensis ......... macrolepidella?

Q. ithaburensis ssp. macrolepis (Ktsch.) Hedge \& Yalt. . basiguttella, macrolepidella, samiatella, zangherii

(= Q. aegilops L., Q. ehrenbergii Kotschy,

Q. macrolepis Kotschy)

Q. petraea (Mattuschka) Liebl.

Q. pubescens Willd. (= Q. humilis Mill. )

Q. pyrenaica Willd.

Q. pyrenaica Willd. ........................ atricapitella, basiguttella, eberhardi
Q. robur L. subsp. robur

atricapitella, basiguttella, dorsiguttella, eberhardi, roborella, ruficapitella, samiatella atricapitella, basiguttella, eberhardi, fasciata, roborella, ruficapitella, samiatella, zangherii la, samiatella, svenssoni

Q. robur subsp. pedunculiflora (K. Koch) Menitsky .. Stigmella sp. (as samiatella) (Popescu-Gorj \& Draghia 1966) (= Q. pedunculiflora K. Koch)

Q. rotundifolia Lam. .................... ilicifoliella, suberivora

(= Q. ilex subsp. ballota (Desf.) Samp. in Bol.,

Q. ilex subsp. rotundifolia (Lam.) T. Morais)

Q. rubra L.

basiguttella, roborella [incidentally]

Q. suber L. .................... eberhardi, ilicifoliella, suberivora, zangherii

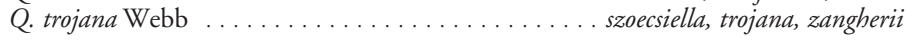

(Wocke, 1862) in the S. ruficapitella group is smaller, and occurs only far away from oaks, in the northern tundra. Moreover, most males of the ruficapitella group have conspicuous androconial scales. For the only fasciate species here treated, see under $S$. fasciata. Females of the uniformly coloured species resemble many other uniform European Stigmella of the $S$. paradoxa, oxyacanthella, thuringiaca, ulmivora and pomella groups.

The genitalia are more characteristic, in particular the presence of a manica, a long aedeagus with many large cornuti of variable size, uncus with two horns, usually far apart and gnathos also with separated horns. Females are usually recognized by a tergum 8 with two furrows and, except the first five species treated here, by a strongly developed accessory sac with spines or other sclerotizations and a reduced or absent corpus bursae.

\section{Description}

Adults uniformly brown, often with shining wingtips, or wings with metallic fascia and bright shining colours; some species with a more extensive pale pattern. Scape sometimes with dark edge, especially in eastern Palaearctic species. Males often with extensive androconial scales on hindwing, either long spatulate scales extending into fringe, or special yellow or blackish scales on wing surface only. Males with long and distinct abdominal tufts, usually inserted on well sclerotized plates.

Male genitalia with bilobed uncus, and bilobed gnathos, rarely with anterior processes. Valva usually with distal pointed process, inner lobe variable. Aedeagus usually with large and spinose manica; vesica with large number of distinct and often large cornuti.

Female genitalia usually with well developed accessory sac, in many species with additional sclerotizations, often in the form of many spines, at cost of an 
almost reduced flimsy bursa; in species with a complete bursa, the accessory sac is heavily folded, without spines. Ductus spermathecae usually clearly coiled (413 convolutions) after a long straight part, in species with reduced bursa entering the accessory sac at the posterior end. Tergum 8 often with two longitudinal depressions.

\section{Biology}

The caterpillars are leafminers, almost exclusively of Fagaceae, in Western Palaearctic one feeds on Fagus (S. hemargyrella (Kollar, 1832)), the 19 species discussed here on Quercus, some of these feed also on Castanea. European Castanea sativa has no monophagous specialists. See table 1 for a survey of the hosts. Most records are from Quercus robur and $Q$. pubescens: eight species feed on each. In Eastern Palaearctic some species feed also on other fagaceous genera: Castanopsis and Lithocarpus. Few species feed on other hosts: Betula (S. tristis), Lonicera (S. lonicerarum and S. monticulella Puplesis, 1984) and Acer (S. speciosa (Frey, 1857) and S. kuznetzovi Puplesis, 1994). Leafmines are invariably gallery mines with a central line of frass of variable width. Larvae vacating the mine before pupation through a slit in the upper epidermis. Larvae spin a tough flat, silken cocoon amongst leaf-litter or in the soil. Mines are illustrated in detail by Borkowski (1972), Johansson \& Nielsen (1990), Patočka (1980) and A. \& Z. Laštúvka (1997) and photographs are provided by Ellis (2003), Dickerson et al. (2003) and Gustafsson (2003). It is not clear in most sources whether the illustrated mines are identified by reared adults, but we think that this is certainly the case in the publications by Borkowski and Johansson \& Nielsen and the website by Gustafsson.

Life-history. - Usually bivoltine, but some species are univoltine in the north and have probably more generations in the south. Adults are active flyers, and are amongst the most frequent Nepticulidae taken during light collecting, often far away from nearest oak trees. For photographs of some live adults see website by Kimber (1999-2003).

\section{Distribution}

In Palaearctic largely following the distribution of oaks (shown in several maps, e.g. fig. 164, 168), which is divided into a western Palaearctic region, with southeast extension into the Elbrus mountains in Iran and to the east reaching the Urals; and an eastern part, from Japan, eastern Siberia (Primorye) and China westwards in a narrow zone along the Himalayas, and reaching East Afghanistan (fig. 167). S. kasyi actually belongs more to the eastern than western Palaearctic fauna; it is described here for convenience. The group also occurs in southeast Asia (positive records from Nepal and Vietnam), but is hardly studied there. Out- side the range of Fagaceae, S. tristis represents the group in arctic regions of Europe, and probably will also be found in Siberia.

\section{Taxonomic history}

The oak mining Stigmella have puzzled all authors prior to Johansson (1971). He was the first to revise all on the basis of their genitalia. Very few genitalia figures had been published before 1971. Johansson treated ten Quercus feeding species and S. tristis (Wocke, 1862), which feeds on Betula. Since then, three more oak-mining species were described (Borkowski 1972, Klimesch 1978, Z. \& A. Laštu̇vka 1998). Johansson also recognized a $S$. hemargyrella group (for one species), which was enlarged by Van Nieukerken (1986b) to include the non-Quercus feeding species $S$. hemargyrella (on Fagus), S. speciosa (on Acer) and S. lonicerarum (on Lonicera). Johansson \& Nielsen (1990) combined the S. hemargyrella and ruficapitella groups tentatively into one $S$. ruficapitella group, because monophyly of the hemargyrella group seemed doubtful. Kemperman et al. (1985) described the Japanese species on oak, and divided them into a $S$. suberivora group and S. ruficapitella group, mainly based on the female genitalia and also recognized a new S. caesurifasciella group. Puplesis (1994) and Diškus \& Puplesis (2003), recognized the S. hemargyrella group, S. ruficapitella group and S. castanopsiella group. Van Nieukerken \& Liu (2000) revised the Eastern Palaearctic oak-feeding species, and recognized thirteen species, twelve in an enlarged $S$. ruficapitella group and one in the $S$. caesurifasciella group.

Here we also view the group in the wide sense of Van Nieukerken \& Liu (2000). Apart from the Quercus feeding species, it also includes in the western Palaearctic S. tristis, feeding on Betula, S. hemargyrella (on Fagus), S. speciosa, S. kuznetsovi (both on Acer) and S. lonicerarum (on Lonicera). These were fully treated by Johansson \& Nielsen (1990) or Puplesis (1994).

\section{Checklist of western Palaearctic Quercus feeding Stigmella}

Stigmella Schrank, 1802

S. ruficapitella group

1. S. fasciata sp. $\mathbf{n}$.

2. S. suberivora (Stainton, 1869) ......... 322

3. S. ilicifoliella (Mendes, 1918) comb. n., stat. rev. . . . . . . . . . . . . . . . . 326

4. S. cocciferae sp. n. ................ 329

5. S. kasyi sp. n. ..................... 331

6. S. basiguttella (Heinemann, 1862) ...... 332

7. S. macrolepidella (Klimesch, 1978) ...... 333

8. S. svenssoni (Johansson, 1971) . ........ 334

9. S. szoecsiella (Borkowski, 1972) ......... 335

10. S. zangherii (Klimesch, 1951) ......... 336 

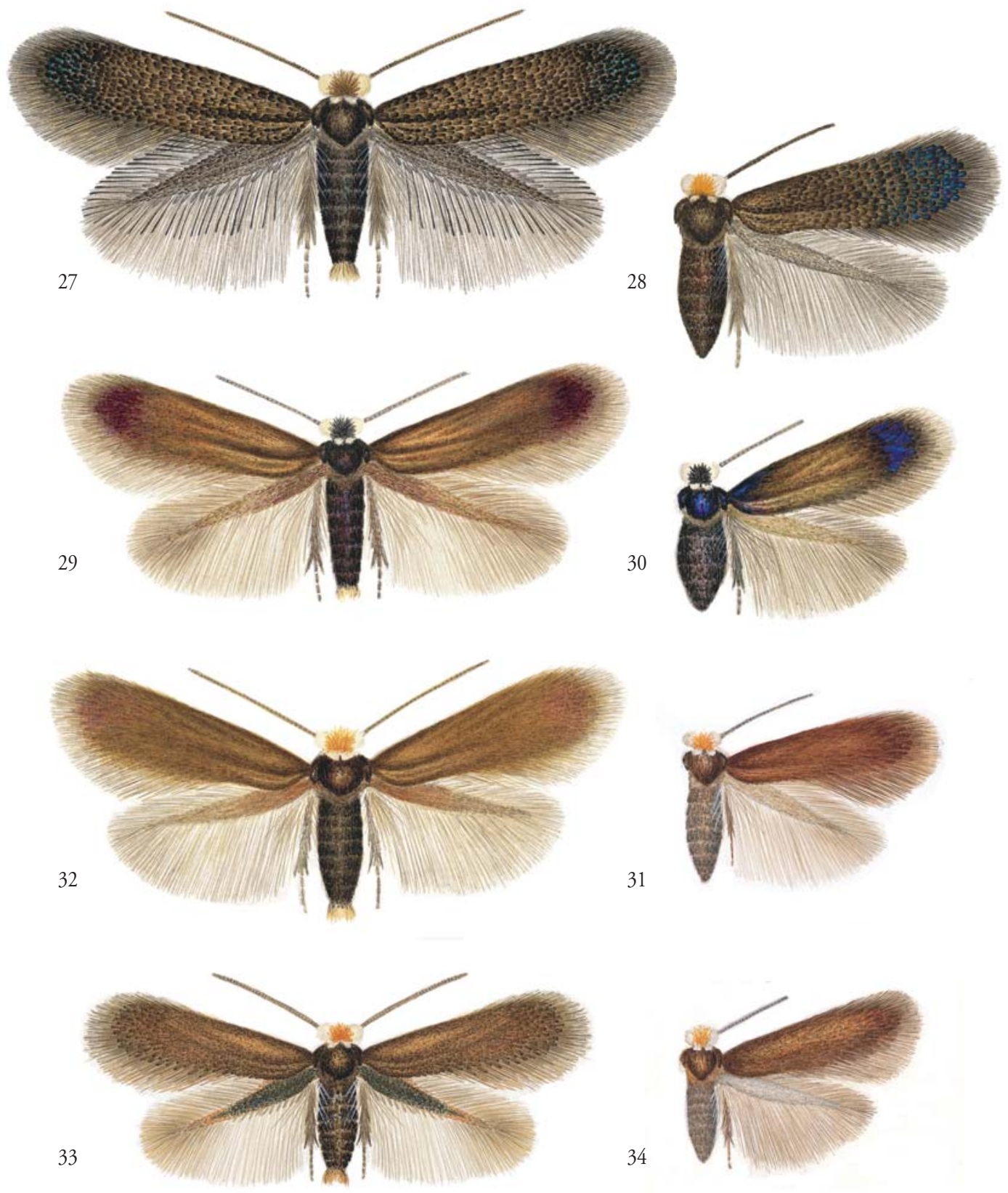

Figs. 27-34. Stigmella spp., dorsal aspect. - 27, S. karsholti ơ, paratype; 28, S. karsholti + , paratype; 29, S. samiatella, ơ, Sweden, Högsby; 30, S. samiatella, + , Sweden, Kullaberg; 31, S. samiatella, o (red-headed), Greece, Kos, Asfendiou; 32, S. ro-

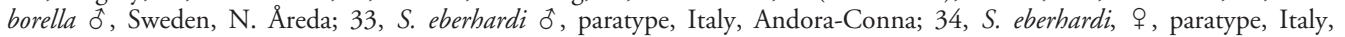
Castelforte. In figs. 27 and 34 the left wings are digitally mirrored right wings. All on same scale, ca $13 \times$. 

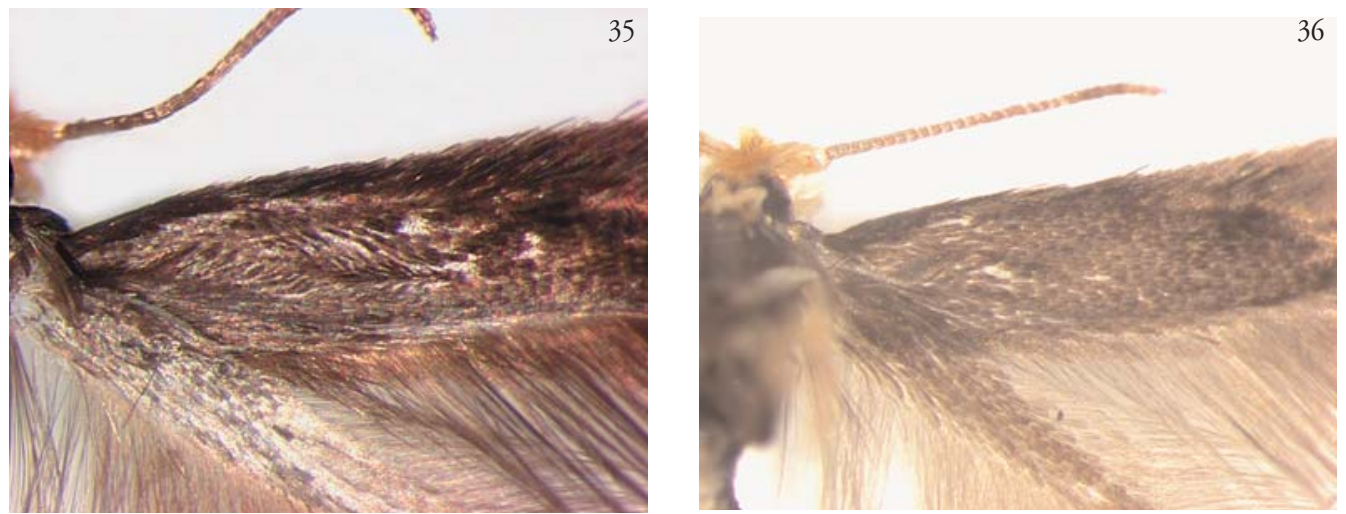

Figs. 35-36. Stigmella spp., male forewing underside. - 35, S. svenssoni, Sweden, Sandhammaren, showing special scales in 'fish-bone' pattern; 36, S. roborella, Netherlands, Winterswijk, scale cover 'normal'.

11. S. dorsiguttella (Johansson, 1971) ...... 339

12. S. trojana Z. \& A. Laštúvka, 1998 ...... 339

13. S. bicuspidata sp. n. ................ 341

14. S. ruficapitella (Haworth, 1828) ....... 342

15. S. atricapitella (Haworth, 1828) . ...... 342

16. S. karsholti sp. n. ................. 343

17. S. samiatella (Zeller, 1839) .......... 345

18. S. roborella (Johansson, 1971) ......... 347

19. S. eberhardi (Johansson, 1971) ........ 349

\section{Key to males}

1. Forewing with distinct narrow silvery fascia; scape edged bluish grey; underside of forewing with a reflexed row of spatulate metallic androconial scales along dorsum 1.S. fasciata

- Forewing without distinct fascia, colour pattern absent or very indistinct, not metallic; scape not edged except in one unicolorous species from Afghanistan; such androconial scales lacking ........2

2. Hindwing with distinct spatulate androconial scales extending into costal and dorsal fringe, at least half length of dorsal cilia.

- Hindwing without distinct and long androconial scales; if androconial scales are present, they are confined to the disk of the hindwing and only extend a short distance into the fringe, or there are narrow spatulate scales along costa only .............. 9

3. Frontal tuft dark brown or black ........................ 4 Frontal tuft yellow or yellowish orange ............. 7

4. Scape edged with bluish grey, large species from Afghanistan 5. S. kasyi Scape uniformly white, European or Mediterranean species 5

5. Forewing coarsely scaled, strongly shining bronze brown with purplish iridescence. Androconial scales relatively narrow and inconspicuous
Forewing smoothly scaled, much darker golden brown, iridescence less conspicuous. Androconial scales contrasting black and relatively wide ........ 6

6. Androconial scales at most one third length of cilia, forming triangular patch in dorsal cilia, antenna with 30-35 segments ......... 14. S. ruficapitella

- Androconial scales longer, at most two-thirds length of cilia, antenna with 30-35 segments .......

15. S. atricapitella

$=$ Androconial scales longer, at most two-thirds length of cilia, antenna with 39-50 segments ...... 3. S. ilicifoliella [rare dark headed form]

7. Androconial scales not reaching tip of hindwing. Eastern Mediterranean species ....... 4. S. cocciferae

- Androconial scales reaching tip of hindwing. Western Mediterranean species .............................. 8 [Note: pale-headed karsholti may key out here, check couplet 5.]

8. Male genitalia very broad, valvae bulging, often visible without dissection. In all western Mediterranean countries 2. S. suberivora

- Male genitalia not extremely broad, valvae not bulging; in southern France and Iberian Peninsula …....................................... 3. S. ilicifoliella

9. Forewings with more or less extensive pale pattern, occasionally completely pale, sometimes pattern reduced to a basal spot or dorsal stripe .............. 10

- Forewings completely unicolorous dark .......... 14

10. Hindwings distinctly yellow or brown, covered with small androconial scales; frontal tuft pale or

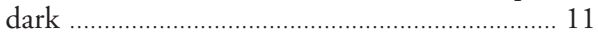

- Hindwings usually grey or pale ochreous yellow, no androconial scales or very few; frontal tuft black 12

11. Frontal tuft pale. Pattern on forewing usually only a narrow dorsal stripe. Hindwings either yellow or brown 11. S. dorsiguttella 
- Frontal tuft dark, often black. Pattern on forewing variable, usually some pale spots. Hindwings distinctly yellow to almost cream white 10. S. zangherii

12. Forewing primarily white with some dark spots; frontal tuft brownish to black. Abdomen pale, almost white. Only known from eastern Mediterranean ............................... 7.S. macrolepidella

- Forewing primarily dark with variable extension of pale pattern, usually confined to basal spot or dorsal stripe. Abdomen darker 13

13. Forewing with variable pattern, usually a basal spot present. Hindwing grey, without spatulate scales. Widespread species .......... 6. S. basiguttella

- Forewing dark, along dorsum pale, no basal spot. Hindwing ochreous yellow; along costa with narrow spatulate scales (often hidden under forewing). Eastern Mediterranean species

11. S. trojana

14. Frontal tuft dark, at least on vertex, usually black

F............................................................. 15

- Frontal tuft yellow or yellowish orange ........... 19

15. Hindwing with deep yellow or ochreous yellow androconial scales, forewing underside with distinct yellow patch ….................... 10. S. zangherii

- Hindwing grey or brownish, without androconial scales or with rather indistinct ones, forewing underside without such patch ............................. 16

16. Hindwing along costa with narrow spatulate scales (often hidden under forewing), species of Greece and Turkey 17

- Hindwing costa without spatulate scales, widespread species ............................................... 18

17. Hindwing greyish. Forewing usually with a pale stripe along dorsum, underside with basal pale area …........................................... 12. . trojana

- Hindwing bronze brown. Forewing variable, underside covered with brown scales, no pale area .. 13. S. bicuspidata

18. Forewing usually with indications of a pale basal spot and paler area in middle, hindwing grey ...... 6. S. basiguttella

- Forewing uniform dark. Hindwing distinctly brown .................................... 17. S. samiatella

19 . Hindwing completely pale grey without any androconial scales ............................................... 20

- Hindwing yellow or brownish, covered with minute androconial scales ................................. 21

20. Forewing underside with thin layer of scales arranged in 'fish-bone' pattern (fig. 35). Large species, forewing length $2.8-3.3 \mathrm{~mm}$; hindwing without androconial scales ............. 8. S. svenssoni

- Forewing underside with normal layer of darker scales, all directed towards tip (fig. 36); mediumsized species, forewing length $2.2-3.1 \mathrm{~mm}$; hindwing usually with (not very conspicuous) brown androconial scales

18. S. roborella

- Forewing underside with normal layer of darker scales, all directed towards tip; smaller species, forewing length 2.1-2.3 $\mathrm{mm}$; hindwing without androconial scales 9. S. szoecsiella

21. Hindwing with thick cover of distinctly yellow, ochreous yellow or pale brown androconial scales. Forewing underside with patch of similar scales to $2 / 3$. Unicolorous form of: .......... 11. S. dorsiguttella

- Hindwing brown or dark bronze. Forewing underside with uniformly dark scale cover .......... 22

22. Hindwing with distinct dark bronze androconial scales with bluish iridescence, tip of hindwing yellowish brown; spatulate scales absent

19. S. eberhardi

[Note: on Sicily S. roborella may have very similar androconials.]

- Hindwing bronze brown; costa with narrow spatulate scales ...............................13. S. bicuspidata

$=$ Hindwing brown, androconial scales not very conspicuous; wingtip concolorous; spatulate scales absent ................................18. S. roborella or rare pale-headed 17. S. samiatella

\section{Identification of females}

Because the females of many species can be extremely similar, checking of genitalia is usually needed. Instead of providing a key, we give some external characters for initial sorting. The female of bicuspidata is unknown.

Head with dark frontal tuft:

6. S. basiguttella, 7. macrolepidella, 10. zangherii, 12. trojana, 15. atricapitella, 17. samiatella.

Head with pale yellow or orange frontal tuft:

1. S. fasciata, 2. suberivora, 3. ilicifoliella, 4. cocciferae, 5. kasyi, 8. svenssoni, 9. szoecsiella, 11. dorsiguttella, 14. ruficapitella, 16. karsholti, southern European 17. samiatella, 18. roborella, 19. eberhardi.

Silver fascia present:

$$
\text { 1. S. fasciata. }
$$

Pale non-metallic pattern often present:

6. S. basiguttella, 7. macrolepidella, 10. zangherii, 11. dorsiguttella, 12. trojana.

Abdomen ending in rather pointed ovipositor:

7. S. macrolepidella, 18. S. roborella.

\section{Key to male genitalia}

1. Aedeagus basally considerably widened; vesica curved, bearing extremely long curved cornuti in basal part, following curvature of vesica (figs. 65, $67,69,72,75,96,102,104,108,110,112)$.... 2 
- Aedeagus not or slightly widened in basal part; vesica without distinct curvature or curved in zigzag pattern, cornuti not long and curved .... 6

2 . Uncus with posterior processes very close together (fig. 66) 10. S. zangherii

- Uncus with posterior processes widely apart ......3

3. Aedeagus with spinose manica present; aedeagus about $1.5 \times-1.6 \times$ as long as wide (fig. 65)

9. S. szoecsiella

- Aedeagus without spinose manica; aedeagus twice as long as wide or longer .......................... 4

4. Valva with inner lobe distinctly bicuspidate and pointed (figs. 74, 76) 13. S. bicuspidata

- Valva with inner lobe rounded .. 5

5. Uncus lobes narrow, widely apart; valva with short distal process, inner lobe exceeding it (figs. 68-70); yellow or brown androconial scales on hindwing and forewing underside very conspicuous 11. S. dorsiguttella

- Uncus lobes almost triangular, approximated; distal process of valva exceeding inner lobe (figs. 7173); androconial scales on hindwing or forewing underside hardly visible 12. S. trojana

6. Uncus lobes extremely widely separated, bearing many long setae; gnathos also with widely separated processes, joined by very thin bar, longer than one process. Overall genitalia aspect very different from other species (figs. 40-43, 90) ....... 1. S. fasciata

- Uncus lobes not so far apart, without setae; gnathos processes separated by bar of normal thickness, shorter than one process 7

7. Vesica basally with a curved band of broad spiny cornuti with serrate bases; manica very large, covering $2 / 3$ to complete aedeagus (figs. $44,47,49$ ) .... 8

- Cornuti in vesica never with serrate bases; manica usually not so large ........................................ 10

8. Manica covering aedeagus almost completely .... 9

- Manica covering distal 2/3 of aedeagus; capsule and valvae not extremely widened (figs. 48, 49, 52)

4. S. cocciferae

9. Capsule and valvae extremely broad; valva externally with field of small papillae (figs. 44, 45, 50) 2. S. suberivora

- Capsule and valvae not extremely broad; valva with field of hairlike setae (figs. 46, 47, 51) 3. S. ilicifoliella

10. Manica ventrally with a large round patch of hundreds of papillate setae (figs. 54, 55); capsule and valvae very broad ......................... 5. S. kasyi

- Manica, if present, with rather indistinct fine spines; capsule usually not particularly broad ....... 11

11. Aedeagus very long (more than $550 \mu \mathrm{m}$ ) and cylindrical, about twice as long as capsule
- Aedeagus much shorter (less than $560 \mu \mathrm{m}$ ), 1$1.5 \mathrm{x}$ as long as capsule; cylindrical or anteriorly widened

12. Manica absent; vesica with very long cornuti from base almost to tip, surrounded by many smaller cornuti; at posterior tip of aedeagus a few strong spines. Capsule relatively narrow; inner lobe of valva regularly curved (figs. 97, 98)

6. S. basiguttella

- Distinct spinose manica present; anterior half of aedeagus only with small blunt cornuti, posterior half with slightly longer spinose cornuti, at tip a blunt lobe. Capsule broader; valva with inner lobe pronounced (figs. 81, 82, 115, 116)

16. S. karsholti

13. Aedeagus with three groups of cornuti which are widely separated (figs. 117, 119) 17. S. samiatella

- Aedeagus almost completely filled with cornuti, sometimes in different groups, but without empty space between ............................................ 14

14. Cornuti in aedeagus in three well defined, separated groups of different shape, on a more or less contorted vesica in a zigzag pattern (figs. 121, 125); aedeagus anteriorly distinctly widened ............. 15

- Cornuti filling aedeagus almost completely; different forms overlapping; aedeagus anteriorly not or hardly wider than posteriorly ...................... 16

15. Valva with inner lobe pointed, resulting in a valva with two pointed ends; aedeagus with slight curvature in vesica (figs. 120-123) ....... 18. S. roborella

- Valva with inner lobe rounded; aedeagus with distinctly contorted vesica (figs. 87, 88, 124, 125)

19. S. eberhardi

16. Aedeagus anteriorly with a few straight and long cornuti of $1 / 3$ to $1 / 2$ aedeagus length ................ 17

- Aedeagus anteriorly with small cornuti, longest cornuti (posteriorly) not more than $1 / 4$ aedeagus length

18

17. Aedeagus anteriorly with 4 strong, slightly curved, cornuti of $1 / 2$ aedeagus length; uncus lobes each bilobed (figs. 61-63, 99)

7. S. macrolepidella

- Aedeagus anteriorly with several straight cornuti of at most $1 / 3$ aedeagus length; uncus lobes simply pointed (figs. 100, 101)

8. S. svenssoni

18. Manica conspicuous, almost twice as wide as aedeagal tube. Aedeagus posteriorly with a single big tooth at right side; about 30-40 long cornuti at left side (figs. 105, 106) ..... 14. S. ruficapitella

- Manica inconspicuous, only slightly wider than aedeagal tube. Aedeagus posteriorly with a big tooth accompanied by several smaller ones at right side; about 15-20 long cornuti at left side (figs. 113, 114) 15. S. atricapitella 


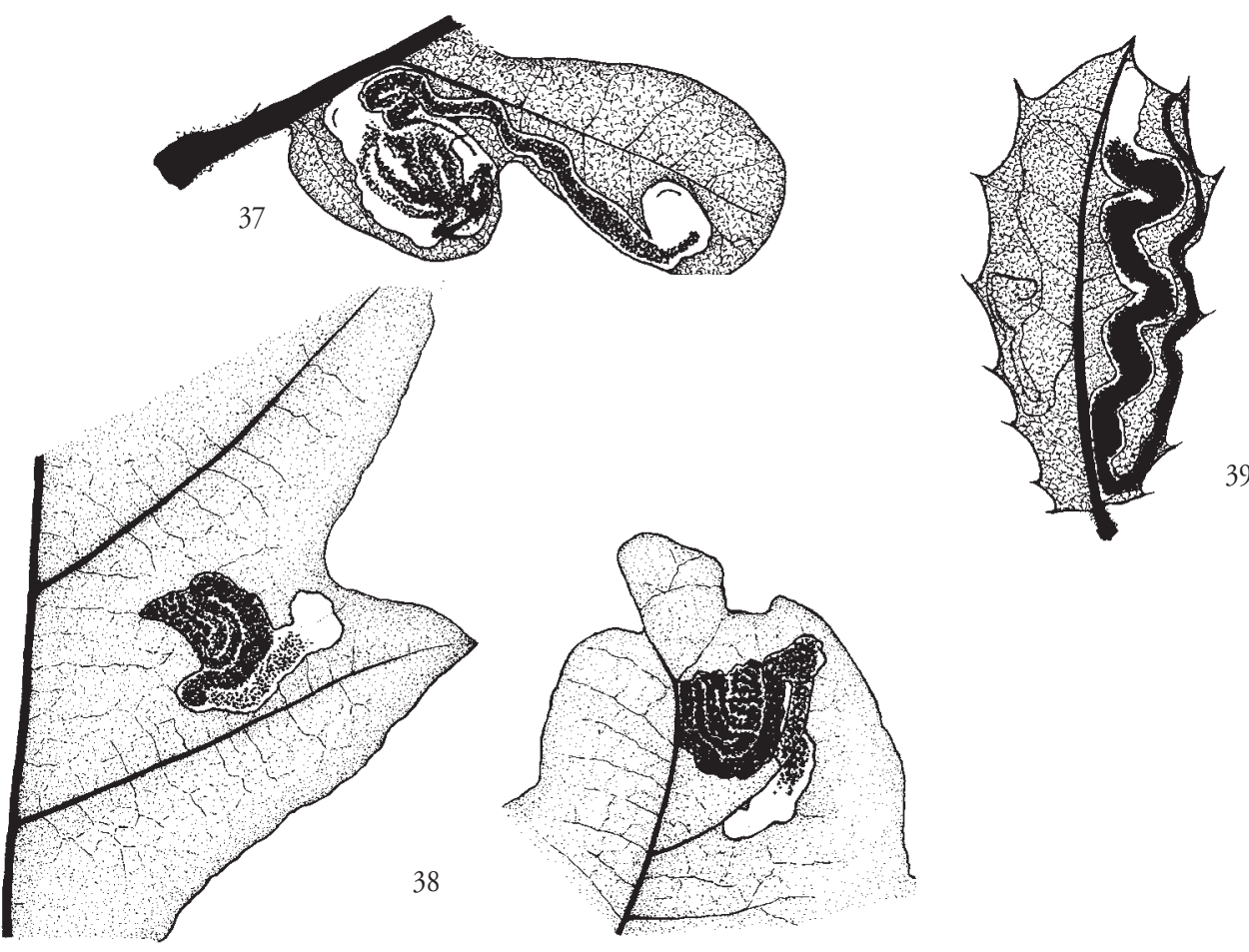

Figs. 37-39. Leafmines of Stigmella spp. - 37, S. fasciata, two intertwined mines, Quercus pubescens, Slovenia, from these holotype was reared; 38, S. fasciata, two mines on Quercus cf. pubescens, Greece, Parnis Oros; 39, S. cocciferae, mine on Quercus coccifera, on left side old mine of Ectoedemia cf. caradjai (Groschke).

\section{Key to female genitalia}

Note: female of $S$. bicuspidata unknown.

1. Bursa consisting of a distinct corpus bursae and a more heavily sclerotized and folded accessory sac (figs. 56-60); no spines or sclerotizations in bursa

.. 2

- Bursa consisting of a heavily sclerotized accessory sac with spines and or other sclerotizations (figs. 77-80, 131-145); corpus bursae absent or reduced to flimsy unsclerotized sac, usually disappearing during preparation

2. Ductus spermathecae with extremely long straight part (exceeding bursa length), at most 2 convolutions (fig. 56) …………....... 1. S. fasciata

- Ductus spermathecae with shorter straight part and 4 or more convolutions

3. Bursa $2-4 \times$ as long as accessory sac; ductus spermathecae with 4.5-6.5 convolutions (figs. 5759); European species

- Bursa less than $1.5 \times$ as long as accessory sac; ductus spermathecae with 6 convolutions (fig. 60); species from Afghanistan

5. S. kasyi

4. Ductus spermathecae with ca 6.5 convolutions, ratio bursa /accessory sac 3.5-3.8 …2. S. suberivora
- Ductus spermathecae with 4.5 convolutions, ratio bursa /accessory sac 2.3-3 …........ 3. S. ilicifoliella

$=$ Ductus spermathecae with 6-7 convolutions, ratio bursa /accessory sac 2.4-2.6 .......... 4. S. cocciferae

5. Accessory sac with large sclerotized plate and several small triangular spines (fig. 133)

8. S.svenssoni

- Accessory sac with spines only, usually larger and of different sizes .............................................. 6

6. Spines in accessory sac confined to posterior half

- Spines covering accessory sac completely, or leaving only small portion anteriorly open ............ 12

7. Spines in two groups inserted on two bulbous lobes, pointing anteriorly; all spines of about similar length (figs. 80, 138) ............... 12. S. trojana

- Spines dispersed, not in two groups on lobes; if on lobes then spines of different sizes present ......... 9

9. Ductus bursae distinctly coiled (figs. 144, 145) ..

- Ductus bursae not coiled, accessory sac distinctly elongate, $2 \times$ as long as wide or longer (figs. 139, 140)

$=$ Ductus bursae not coiled, accessory sac almost 
globular (figs. 78, 134)

9. S. szoecsiella

10. Ductus with about one complete coil; anteriorly spines slightly longer than posteriorly (fig. 144); abdominal tip (ovipositor) pointed ... 18. S. roborella

- Ductus forming two coils; different spines throughout (figs. 89, 145); abdominal tip wider

19. S. eberhardi

11. All spines small, not on a rounded lobe; a small chitinized plate posteriorly (fig. 139)

14. S. ruficapitella

- Spines of different length, the longer ones on a rounded lobe; accessory sac anteriorly with some more or less sclerotized folds, no chitinized plate (fig. 140)

15. S. atricapitella

12. Small spines arranged on a long narrow coiled band; anteriorly also an extra group of spines (figs. 85, 86) ............................ 17. S. samiatella

- Spines not on such a band, when coiled than spines longer

12

13. Ductus spermathecae with many convolutions, more than 10 ; accessory sac with spines all over distal $2 / 3$, a dense band at right hand side in dorsal view (figs. 83, 84) ........................ 16. S. karsholti

- Ductus spermathecae with up to 5 convolutions; accessory sac with less cover by spines ............. 14

14. Four species with different distribution of spines and shape of accessory sac, check figures: ..fig. 131, 6. S. basiguttella fig. 136, 11. S. dorsiguttella figs. 77, 132, 7. S. macrolepidella ..figs. $79,135,10$. S. zangherii

\section{Stigmella fasciata sp. n.}

(figs. 1, 2, 37, 38, 40-43, 56, 90, 126, 146, 164)

Type material. - Holotype 9 , SLOVENIA: $7 \mathrm{~km} \mathrm{SE}$ Piran: Cedle, 300 m, 12.x.1983, J. J. Boomsma \& E. J. van Nieukerken, Quercus pubescens VU no 83444KE2 e.l. 24.v.1984, genitalia slide VU no 1964 (RMNH). - Paratypes 4tô, 19 : GREECE: $2 \hat{0}$, Akhaia, Kalávrita, 20.vi.1997, A. Laštüvka (AL, RMNH); 10 , Evro, Kavisos 100 m, 22-23.viii.1985, A. Moberg (NRMS). - TURKEY, 10 , 19 , Adana, Taurus, $4 \mathrm{~km} \mathrm{NE}$ Feke, 1000 m, 7.ix.1983, Gg. Derra (GD).

Other material: leafmines. - SLOVENIA, 2 mines (of which holotype was reared). - GREECE: 6 vacated mines, Attika, Parnís Oros, $8 \mathrm{~km} \mathrm{~N}$ Dhekélia, 29.ix.1980, Menken \& van Nieukerken, Quercus cf. pubescens (RMNH).

Additional records (A. \& Z. Laštúvka, in litt.). - CROATIA: 2ㅇ, Dalmacia, Oračac, 12.vii.2000 and 23.viii.2001; 25. vii.2003, 3 larvae on $Q$. pubescens; 2001-2003, many empty mines. - GREECE: 10, Lakonia, Krokees, 16.vi.1997; 10 , Arkadia, Pigadakia, 22.vi.1996; 1 ㅇ, Argolis, Skotini, 20.vi. 1998.

\section{Diagnosis}

Differs from all other western Palaearctic oak mining Stigmella by presence of fascia and distinct cilialine, male in addition by peculiar androconial scales on forewing underside and dark edged scape. Female can only be confused with other European Stigmella with fascia and cilia-line: S. freyella (Heyden, 1858), which has a dark head and paler cilia, S. malella (Stainton, 1854), which has a much straighter, unconstricted fascia and $S$. hemargyrella which is much larger. Further the male genitalia are highly diagnostic, not resembling any other species. Female genitalia rather similar to suberivora, ilicifoliella and cocciferae, but these have the ductus spermathecae with more than 4 convolutions, whereas fasciata has only 2 .

\section{Description}

Male (fig. 1). - Forewing length 1.95-2.35 mm, wingspan 4.3-5.0 mm. Head: frontal tuft ochreous, collar dark brown; scape white, edged with dark bluish grey; antenna with 26-29 segments, yellowish grey. Thorax dark copper brown. Forewing: basal half dark copper-brown with weak metallic reflections; fascia postmedial, very narrow, consisting of only a few silvery scales; distal to fascia coarsely scaled, dark copper brown to fuscous; cilia-line distinct; cilia greybrown; underside grey-brown with a long reflexed row of spatulate lead-grey to silver androconial scales along dorsum (fig. 1), covering an elongate pocket. Hindwing: dark brown to fuscous, along costa and dorsum with bluish black androconial scales extending over cilia to at most one third, near frenulum longer and broader; underside of hindwing and androconial scales grey-brown. Abdomen almost black, no visible anal tufts; no sclerotized plates.

Female (fig. 2). - Forewing length 2.05-2.15 mm, wingspan $4.5 \mathrm{~mm}$. Head: frontal tuft ochreous, collar yellowish white [almost missing in holotype]; scape yellowish white. Antenna shining fuscous, with 24? segments. Thorax fuscous. Forewing with basal half fuscous, weakly shining; fascia postmedial, shining silver, constricted in middle; distal to fascia coarsely scaled, fuscous; cilia-line distinct; terminal cilia greybrown; underside dark grey-brown. Hindwing greybrown, with weak bronze reflections; cilia grey-brown; underside dark grey-brown. Abdomen removed for dissection, reconstructed in fig. 2 .

Male genitalia (figs. 40-43, 90). - Measurements: see table 3 . Vinculum anteriorly only slightly concave. Uncus with very widely separated horns with many long curved setae. Gnathos with long, very widely separated posterior processes, connected by a very thin, curved bar. Valva with short distal process, and prominent inner lobe; inner margin almost straight; ventrally a transverse ridge with setae; sublateral processes about $1 / 2$ transtilla length. Aedeagus with vesica in dis- 


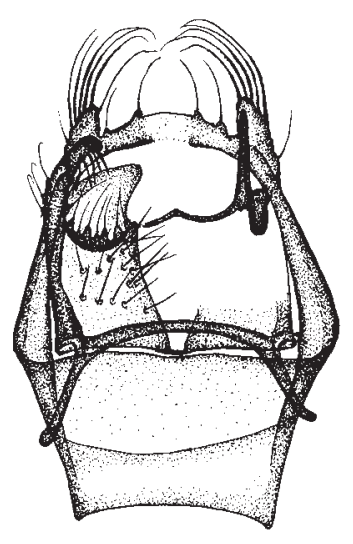

40

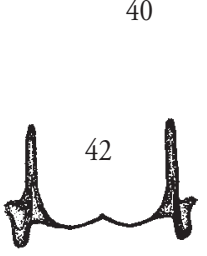

41

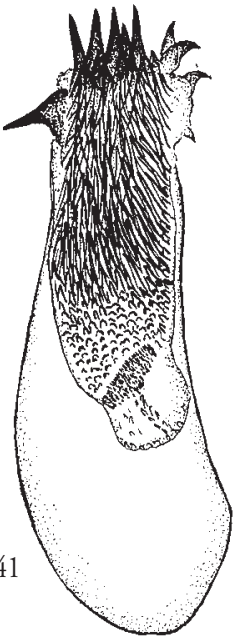

43

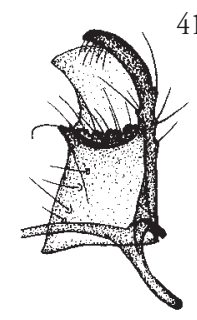

Figs. 40-43. Stigmella fasciata, male genitalia, Turkey, slide EvN 2610. - 40, Capsule; 41, aedeagus; 42, gnathos; 43, valva, dorsal aspect.

tal $2 / 3$ of aedeagus, covered with many needle-shaped cornuti and a group of larger ones distally; manica absent.

Female genitalia (figs. 56, 126, 146). - T8 with two slight indentations laterally, no grooves. Bursa well visible, total length $787 \mu \mathrm{m}$, walls thin, without pectinations. Accessory sac more strongly sclerotized, without pectinations, about $1 / 3$ bursa length. Ductus spermathecae with about 2 convolutions after a long straight part with minute granulation.

\section{Biology}

Hostplant. - Quercus pubescens**.

Leafmine (figs. 37, 38). - Egg on leaf underside, usually 2-3 (or more) eggs are deposited close by, rarely only one. Mine starting as extremely contorted gallery, doubling back several times, closely following earlier track, so that mine forms a brown dot; frass brown and dispersed, almost filling mine completely; last part of mine running away from dot. Not all the eggs hatch or young larvae die, so that in a sample of 86 leaves with mines, A. Laštuivka (in litt.) observed two complete mines in $50 \%$, three in $10 \%$ and only one in $40 \%$ of the leaves. In the case of 2-3 mines, they are usually intertwined closely in the early part (fig. 37 ).

Life-history. - Larvae found in July and October, adults from June to September.
Distribution (fig. 164)

South-east Europe: Slovenia, Croatia, Greece and Turkey.

\section{Remarks}

The male is described on the basis of four specimens, two of which are badly damaged. They are considered to belong to the same species as the female holotype, because both sexes were collected together in Turkey, and clearly belong to the same speciesgroup. We prefer, however, to select the reared female as holotype.

This species is very different from all other Western Palaearctic species in the S. ruficapitella group, but shares several characters with Eastern Palaearctic species (Van Nieukerken \& Liu 2000), which more often have a fascia. The mine is almost indistinguishable from that of $S$. fervida Puplesis, 1984, except that the latter has the egg on leaf upperside and is usually laid singly.

\section{Etymology}

An adjective: fasciatus: with band, because it is the only species in the oak mining group in Europe with a fascia on forewing; here in female form in concordance with female gender of Stigmella.

\section{Stigmella suberivora (Stainton)}

(figs. 3, 4, 44, 45, 50, 57, 91, 127, 147, 165)

Nepticula suberivora Stainton, 1869: 228. Lectotype ô (designated by Johansson 1971: 246): FRANCE [Alpes Maritimes]: Cannes, Quercus suber, yellow larvae, 9.iii.1867, e.l. 8.iv.1867, Stainton (BMNH) [examined].

Nepticula aureocaputella [lapsus] Millière, 1870: 174. Syntypes: FRANCE: Cannes, v and vi, Millière. [probably lost] (synonymized by Van Nieukerken \& Johansson 1987).

Nepticula aureocapitella Millière, 1870: 216 [justified emendation].

Nepticula ilicivora Peyerimhoff, 1871: 413. Lectotype $q$ (designated by Johansson 1971: 248): FRANCE [Alpes Maritimes:] Midi de la France, Quercus ilex, [iii. 1870], Peyerimhoff (MNHN) [examined] (synonymized by Johansson 1971: 246).

Nepticula suberivora; Petersen 1930: 52, fig. 27 (male genitalia), Johansson 1971: 246, Borkowski 1972: 773.

Stigmella suberivora; Beirne 1945: 197, fig. 11 (male genitalia), Emmet 1976a: 241, Johansson \& Nielsen 1990: 227, A. \& Z. Laštủvka 1997: 103.

\section{Diagnosis}

Males characterised by combination of long spatulate androconial scales and pale head colour, but almost inseparable from ilicifoliella and cocciferae. The very broad genitalia with 'bulging' valvae separate suberivora from both; with some experience this can be seen without dissection; otherwise suberivora has significantly fewer antennal segments than ilicifoliella (30-45), with a small overlap, and $S$. cocciferae has no 
Table 2. Measurements of adult Stigmella. Mean, standard deviation and sample size are given in brackets, for sample size 3 or smaller only sample size is provided.

ô forewing length

q forewing length

$\widehat{\delta}$ antennal segments

$\uparrow$ antennal segments

\begin{tabular}{|c|c|}
\hline S. fasciata & $2.0-2.4 \mathrm{~mm}(2.2 \pm 0.2,4)$ \\
\hline S. suberivora & $2.2-3.3 \mathrm{~mm}(2.7 \pm 0.3,19)$ \\
\hline S. ilicifoliella & $2.5-3.0 \mathrm{~mm}(2.7 \pm 0.2,20)$ \\
\hline S. cocciferae & $2.2-3.2 \mathrm{~mm}(2.7 \pm 0.3,25)$ \\
\hline S. kasyi & $3.5 \mathrm{~mm}(\mathrm{I})$ \\
\hline S. basiguttella & I. $8-2.6 \mathrm{~mm}(2.2 \pm 0.2, \mathrm{IO})$ \\
\hline S. macrolepidella & I.8-2.I mm $(2.0 \pm 0.1,5)$ \\
\hline S. svenssoni & $2.8-3.3 \mathrm{~mm}(3 . \mathrm{I} \pm 0.2,5)$ \\
\hline S. szoecsiella & 2.I-2.3 mm (3) \\
\hline S. zangherii & $2.0-2.4 \mathrm{~mm}(3)$ \\
\hline S. dorsiguttella & $2 . \mathrm{I}-2.7 \mathrm{~mm}(2.4 \pm 0.2,7)$ \\
\hline S. trojana & $2 . \mathrm{I}-2.3 \mathrm{~mm}(2.2 \pm 0 . \mathrm{I}, 5)$ \\
\hline S. bicuspidata & $2 . \mathrm{I}-2.2 \mathrm{~mm}(2)$ \\
\hline S. ruficapitella & $2.3-2.8 \mathrm{~mm}(2.6 \pm 0.2, \mathrm{I} 4)$ \\
\hline S. atricapitella & $2 . \mathrm{I}-2.6 \mathrm{~mm}(2.3 \pm \mathrm{O} . \mathrm{I}, \mathrm{I} 4)$ \\
\hline S. karsholti & $2.8-3.3 \mathrm{~mm}(3.0 \pm 0.2,7)$ \\
\hline S. samiatella & $2.4-3.3 \mathrm{~mm}(2.8 \pm 0.3, \mathrm{I} 7)$ \\
\hline borella & 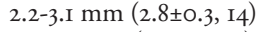 \\
\hline S. eberhardi & $2.3-3.1 \mathrm{~mm}(2.6 \pm 0.3,9)$ \\
\hline
\end{tabular}

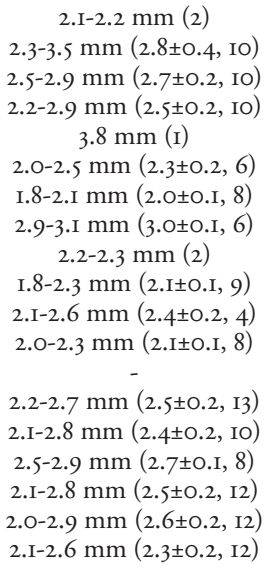

28-29 (2)

$30-45(37.5 \pm 3.7,17)$

$39-50(44 . I \pm 3.2, \mathrm{I} 4)$

$36-49$ (42.8 $3.6,18)$ 53 (I)

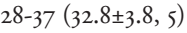

3I-4I (37.I $\pm 3.3,7)$ 33-37 (3)

$33(2)$

$3 \mathrm{I}-38(35.2 \pm 2.7,5)$

$33-35(34.0 \pm 0.8,4)$

34-36 (35.0 $\pm 1.0,5)$ 32 (I)

3O-35 (33.4 \pm I.9, II) $3 \mathrm{O}-35$ (32.I $\pm \mathrm{I} .4, \mathrm{I} 2)$ 37-44 (4I.5 $\pm 2.9,7)$ 28-33 (30.6 \pm I.6, II)

$33-43(38.1 \pm 2.9,9)$

$33-40(36.9 \pm 2.7,7)$
24 ?

$33-40(35.0 \pm 2.4,7)$ $33-40$ (3)

30-37 (33.8 $2.3,9)$ 48 (I)

23-27 (24.6士I.8, 5)

25-3I (27.I \pm I.9, I4)

$25-29(26.8 \pm$ I. 5,6$)$ 25 (I)

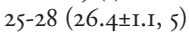
25-26 (3)

$2 \mathrm{I}-26(23.9 \pm \mathrm{I} .8,7)$

23-27 (25.5 \pm I. 5, I2)

$25-29$ (26.3 \pm I. 5,9$)$

3O-33 (3I.O \pm I.I, 8)

24-28 (25.5 \pm I.O, II)

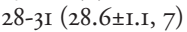

25-3I (26.4 \pm I.9, 9)

Table 3. Measurements of male genitalia of Stigmella. Mean, standard deviation and sample size are given in brackets, for sample size 3 or smaller only sample size is provided.

\begin{tabular}{|c|c|c|c|c|c|c|}
\hline & capsule length & capsule ratio l:w & valva length & aedeagus length & aedeagus l:w & ratio aed:caps 1 \\
\hline S. fasciata & $249-302 \mu \mathrm{m}(2)$ & I.2-I.2 (2) & $\mathrm{I} 44-\mathrm{I} 77 \mu \mathrm{m}(2)$ & $3 \mathrm{I} 6-320 \mu \mathrm{m}(2)$ & $2.4-2.8(2)$ & I.I-I.3 (2) \\
\hline S. suberivora & $\begin{array}{c}295-347 \mu \mathrm{m} \\
(325.9 \pm 24 . \mathrm{I}, 6)\end{array}$ & $\begin{array}{c}0.8 \text {-I.0 } \\
(0.85 \pm 0.07,6)\end{array}$ & $\begin{array}{c}208-238 \mu \mathrm{m} \\
(224.6 \pm 9.9,6)\end{array}$ & $\begin{array}{c}408-44 \mathrm{I} \mu \mathrm{m} \\
(427.2 \pm \mathrm{I} 2.7,6)\end{array}$ & $\begin{array}{c}2.4-2.9 \\
(2.65 \pm 0.18,6)\end{array}$ & $\begin{array}{c}\text { I.2-I.5 } \\
\text { (I.32 } 32.10,6)\end{array}$ \\
\hline S. ilicifoliella & $\begin{array}{c}327-377 \mu \mathrm{m} \\
(348.6 \pm \mathrm{I} 2.8, \text { Io) }\end{array}$ & $\begin{array}{c}\text { I.O-I.2 } \\
\text { (I.I3 } \pm 0.06, \text { IO) }\end{array}$ & $\begin{array}{c}2 \mathrm{II}-254 \mu \mathrm{m} \\
(233.3 \pm \mathrm{I} 7 . \mathrm{I}, \mathrm{IO})\end{array}$ & $\begin{array}{c}380-508 \mu \mathrm{m} \\
(443.2 \pm 34.4, \text { Io })\end{array}$ & $\begin{array}{c}2.4-3.2 \\
(2.84 \pm 0.27, \text { I0 })\end{array}$ & 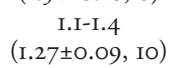 \\
\hline S. cocciferae & $\begin{array}{c}250-385 \mu \mathrm{m} \\
(324.2 \pm 44 . \mathrm{I}, 8)\end{array}$ & $\begin{array}{c}\text { I.O-I.2 } \\
(\text { I.O9 } \pm 0.12,5)\end{array}$ & $\begin{array}{c}215-279 \mu \mathrm{m} \\
(246.8 \pm 22.8,7)\end{array}$ & $\begin{array}{c}448-568 \mu \mathrm{m} \\
(482.5 \pm 39.5,8)\end{array}$ & $\begin{array}{c}2.6-3.5 \\
(3.11 \pm 0.39,5)\end{array}$ & $\begin{array}{c}\text { I. } 4-\mathrm{I} .8 \\
(\mathrm{I} .5 \mathrm{I} \pm 0.18,8)\end{array}$ \\
\hline S. kasyi & $4 \mathrm{IO} \mu \mathrm{m}(\mathrm{I})$ & I.O (I) & $296 \mu \mathrm{m}(\mathrm{I})$ & $549 \mu \mathrm{m}(\mathrm{I})$ & $2.0(\mathrm{I})$ & I. $3(\mathrm{I})$ \\
\hline S. basiguttella & $276-338 \mu \mathrm{m}$ & O.9-I.3 & $218-245 \mu \mathrm{m}$ & $546-656 \mu \mathrm{m}$ & $2.9-3.9$ & I. $8-2.3$ \\
\hline S. macrolepidella & $\begin{array}{c}(299.9 \pm \mathrm{I} 9.5,8) \\
2 \mathrm{IO}-249 \mu \mathrm{m} \\
(233.2 \pm \mathrm{I} 7.0,4)\end{array}$ & $\begin{array}{c}(\mathrm{I} . \mathrm{II} \pm 0.15,7) \\
\text { I.3-I.3 (3) }\end{array}$ & $\begin{array}{c}(23 \mathrm{I} .4 \pm 9 \cdot 3,8) \\
\mathrm{I} 63-207 \mu \mathrm{m} \\
(183.3 \pm 2 \mathrm{I} .7,4)\end{array}$ & $\begin{array}{c}(597.5 \pm 47.4,7) \\
313-369 \mu \mathrm{m} \\
(339.7 \pm 30.0,4)\end{array}$ & $\begin{array}{c}(3.39 \pm 0.39,6) \\
2.7-3.4 \\
(2.93 \pm 0.31,4)\end{array}$ & $\begin{array}{c}(2.00 \pm 0.14,7) \\
\text { I.3-I.5 } \\
(1.46 \pm 0.07,4)\end{array}$ \\
\hline S. svenssoni & $273-339 \mu \mathrm{m}(3)$ & O.9-I.I (3) & $233-26$ I $\mu \mathrm{m}(3)$ & $364-426 \mu \mathrm{m}(3)$ & I.7-I.9 (3) & I.2-I.4 (3) \\
\hline S. szoecsiella & $268-297 \mu \mathrm{m}(3)$ & I.I-I.3 (3) & $\mathrm{I} 88-225 \mu \mathrm{m}(3)$ & $354-359 \mu \mathrm{m}(3)$ & I. 5 -I. $6(3)$ & I.2-I.3 (3) \\
\hline S. zangherii & $300-319 \mu \mathrm{m}(3)$ & I.I-I.2（3） & $216-236 \mu \mathrm{m}(3)$ & $357-376 \mu \mathrm{m}(3)$ & I. 5 -I. 8 (3) & I.2-I.2（3) \\
\hline S. dorsiguttella & $285-287 \mu \mathrm{m}(2)$ & I.O-I.I (2) & $207-216 \mu \mathrm{m}(2)$ & $436-475 \mu \mathrm{m}(2)$ & $2.2-2.3(2)$ & I.5-I.7 (2) \\
\hline S. trojana & $278-293 \mu \mathrm{m}(2)$ & I.I-I.2 (2) & $207-229 \mu \mathrm{m}(2)$ & $4 \mathrm{I} 3-477 \mu \mathrm{m}(2)$ & 2.0-2.I (2) & I.5-I.6 (2) \\
\hline S. bicuspidata & $257-303 \mu \mathrm{m}(3)$ & I. 2 -I. $3(2)$ & $\mathrm{I} 88-206 \mu \mathrm{m}(3)$ & $398-460 \mu \mathrm{m}(3)$ & I.9-2.I (2) & I. 5 -I. $6(3)$ \\
\hline S. ruficapitella & $\begin{array}{c}305-327 \mu \mathrm{m} \\
(318.9 \pm 9.7,4)\end{array}$ & I.I-I.I (3) & $\begin{array}{c}193-222 \mu \mathrm{m} \\
(2 \mathrm{II} .2 \pm 13.3,4)\end{array}$ & $\begin{array}{c}437-487 \mu \mathrm{m} \\
(468 . \mathrm{I} \pm 22 . \mathrm{I}, 4)\end{array}$ & $2.6-2.8(3)$ & I.3-I.5 (3) \\
\hline S. atricapitella & $240-307 \mu \mathrm{m}(3)$ & $0.9-1.2(3)$ & $\mathrm{I} 62-207 \mu \mathrm{m}(3)$ & $304-4 \mathrm{I} 2 \mu \mathrm{m}(3)$ & $2.0-3 \cdot 3(3)$ & I.3-I.4 (3) \\
\hline S. karsholti & $300-352 \mu \mathrm{m}(3)$ & $0.9-I .2(2)$ & $2 \mathrm{I} 9-246 \mu \mathrm{m}(3)$ & $658-696 \mu \mathrm{m}(3)$ & $3 \cdot 7-3 \cdot 9(3)$ & I.9-2.2(3) \\
\hline S. samiatella & $\begin{array}{c}26 \mathrm{I}-323 \mu \mathrm{m} \\
(302.8 \pm 25.2,5)\end{array}$ & $\begin{array}{c}\text { I.O-I.3 } \\
\text { (I.I4 } \pm \text { O.I2, 5) }\end{array}$ & $\begin{array}{l}207-227 \mu \mathrm{m} \\
(222.5 \pm 8.5,5)\end{array}$ & $\begin{array}{c}475-554 \mu \mathrm{m} \\
(5 \mathrm{I} 8.2 \pm 34.4,5)\end{array}$ & $\begin{array}{c}4 . \mathrm{I}-6.0 \\
(4.78 \pm 0.89,4)\end{array}$ & $\begin{array}{c}\text { I. } 5-2 . I \\
\text { (I. } 73 \pm 0.26,5)\end{array}$ \\
\hline S. roborella & $\begin{array}{c}283-320 \mu \mathrm{m} \\
(297 \cdot 9 \pm \mathrm{I} 4 \cdot 6,6)\end{array}$ & $\begin{array}{c}\text { I.O-I.3 } \\
\text { (I.I6 } \pm \text { O.IO, 6) }\end{array}$ & $\begin{array}{c}\text { I9I-232 } \mu \mathrm{m} \\
(2 \mathrm{II} .5 \pm \mathrm{I} 3.4,6)\end{array}$ & $\begin{array}{c}44 \mathrm{I}-48 \mathrm{I} \mu \mathrm{m} \\
(458.8 \pm \mathrm{I} 6.8,6)\end{array}$ & $\begin{array}{c}2 . \mathrm{I}-2.8 \\
(2.47 \pm 0.32,4)\end{array}$ & $\begin{array}{c}\text { I.4-I.7 } \\
\text { (I.54 }\end{array}$ \\
\hline S. eberhardi & $\begin{array}{c}265-294 \mu \mathrm{m} \\
(280.5 \pm 9.3,9)\end{array}$ & $\begin{array}{c}\text { I.O-I.3 } \\
\text { (I.I3 } \pm \text { O.II, 7) }\end{array}$ & $\begin{array}{l}\mathrm{I} 83-204 \mu \mathrm{m} \\
(\mathrm{I} 92.5 \pm 7.5,9)\end{array}$ & $\begin{array}{c}436-501 \mu \mathrm{m} \\
(458.5 \pm 20.1,9)\end{array}$ & $\begin{array}{c}2.2-2.6 \\
(2.40 \pm 0.14,8)\end{array}$ & $\begin{array}{c}\text { I.5-I.8 } \\
(\text { I. } 64 \pm 0.08,9)\end{array}$ \\
\hline
\end{tabular}



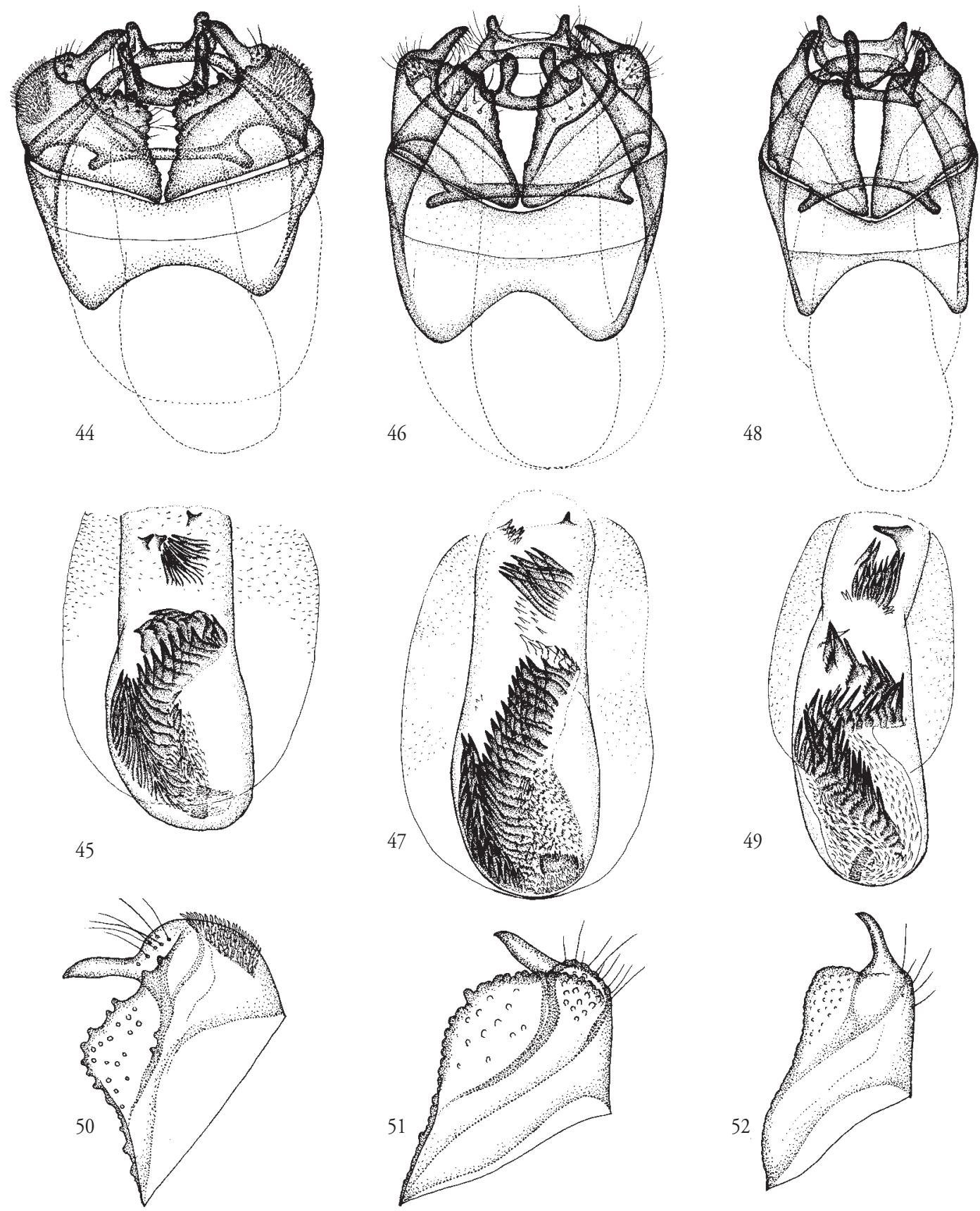

Figs. 44-52. Stigmella spp., male genitalia, capsule $(44,46,48)$, aedeagus $(45,47,49)$ and valva, dorsal aspect $(50-52)$. - 44, 45, 50, S. suberivora, Italy, slides RJ2278+1758 (50); 46, 47, 51, S. ilicifoliella, Spain, slide RJ1754; 48, 49, 52, S. cocciferae, Greece, Kos, slide RJ 1725. 
androconial scales in distal 1/5 of hindwing.

Females very similar to all other pale-headed species, including some outside this group.

Male genitalia with capsule broader than long, valvae bulging and with field of papillae externally. Female genitalia with ductus spermathecae with ca 6.5 convolutions, ratio bursa /accessory sac 3.5-3.8, larger than in ilicifoliella.

\section{Description}

Male (fig. 3). - Forewing length 2.2-3.3 mm, wingspan 4.8-7.1 mm. Head: frontal tuft from almost white or ochreous to yellowish orange, collar pale grey to dark grey-fuscous, occasionally a few yellowish scales. Scape pale yellow. Antenna slightly darker, 3/5 of forewing length, with 30-45 segments, ratio to forewing length 12-16 segments $/ \mathrm{mm}$. Thorax and forewing unicolorous dark bronze brown, at base and on thorax almost bluish black in fresh specimens, a faint purplish lustre at apex; terminal cilia concolorous, underside bronze-brown. Hindwing coarsely scaled, dark grey-brown, dorsum with long dark brown to bluish black androconial scales, extending into fringe, the longest near base, approximately $3 / 4$ of cilia length; along basal half of costa long curved, spatulate bluishblack androconial scales, the longest as long as or longer than the shortest dorsal androconial scales; underside grey brown, cilia dark grey. Abdomen dark grey brown, with conspicuous yellowish to orange anal tufts inserted on well sclerotized plates, and very broad genital capsule, easily visible without dissection.

Female (fig. 4). - Forewing length 2.3-3.5 mm. Antenna with 33-40 segments, ratio to forewing length 11-14 segments $/ \mathrm{mm}$. As male, but collar yellowish white to pale yellow, hindwing grey, androconial scales absent, abdomen dark grey-brown, without anal tufts.

Male genitalia (figs. 44, 45, 50, 91). - Measurements: see table 3. Capsule very wide, wider than long or just as wide as long. Vinculum anteriorly slightly bilobed. Uncus with widely separated horns. Gnathos with widely separated posterior processes, often converging towards tip. Valva with pointed distal process less than $1 / 3$ valva length, pointing inwards, inner margin forming a distinct lobe, ventral outer lobe bulging and covered with field of densely placed minute papillae; sublateral processes very short, or almost absent. Aedeagus with vesica with a more or less curved group of cornuti in basal half; this band comprises about 2030 large cornuti with serrated bases and many small spinelike cornuti; more distally there is a group of ca 10-12 long needle-shaped cornuti and one or a few larger near phallotrema; manica covering aedeagus almost completely, except anteriormost part, covered with small spines and pectinations.

Female genitalia (figs. 57, 127, 147). - T8 with ca
18-23 setae. Bursa length 1170-1606 $\mu \mathrm{m}, 3.5-3.8 \times$ as long as accessory sac. Bursa distally covered with minute pectinations, difficult to see (observed with Differential Interference Contrast only); accessory sac anteriorly with pectinations only. Ductus spermathecae with $6.5(n=3)$ convolutions.

\section{Biology}

Host plants. - Quercus coccifera**, Q. ilex**, Q. rotundifolia**, Q. suber**.

Leafmine. - Egg deposited on leaf upperside. Mine a broad gallery, filled with frass, usually transparent, but in very thick leaves difficult to see. Usually much wider and longer than the gallery mines of Ectoedemia species (E. ilicis (Mendes, 1910), E. haraldi (Soffner, 1942), E. heringella (Mariani, 1939) and E. algeriensis Van Nieukerken, 1985), which differ also by the larvae feeding with venter upwards and showing their ganglia and differently shaped headcapsule. Larva of $S$. suberivora yellow, and with dorsum upwards as all other species in this group. For a detailed description see Gustafsson \& Van Nieukerken (1990).

Life-history. - Larvae have been collected from December to April and in Britain again in July-August, bivoltine, but more generations in Mediterranean possible. Adults have been collected from April to October.

\section{Distribution (fig. 165)}

Widespread in western Mediterranean: Portugal, Spain, Southern France, along Atlantic coast northwards to France: Bretagne, Italy, Corsica, Sardinia, Sicily (new record), Adriatic coast: Slovenia (A. \& Z. Laštüvka 1997), Croatia, Bosnia (Skala 1937) and in North Africa in Algeria and Tunisia (new records); introduced and established in southern England. Records of leafmines in Mallorca (J. Klimesch in litt.) probably belong to this species. To be expected in Morocco and Malta. Occurs from sea level up to 1700 $\mathrm{m}$ in Aurès, Algeria.

\section{Remarks}

Because of the discovery of a second species, hidden amongst material of $S$. suberivora ( $S$. ilicifoliella, see below), the identity of the various synonyms of suberivora had to be re-evaluated. The male lectotype of $S$. suberivora was illustrated by the junior author (Johansson 1971) and there is no doubt about its identity. The lectotype of Nepticula ilicivora Peyerimhoff is a poorly preserved female, with a genitalia slide; however, the slide does not show the main distinguishing character, the ductus spermathecae. Overall it agrees better with suberivora, and male specimens in the old French collections in Paris, labelled as ilicivora are all suberivora. Nepticula aureocapitella Millière was synonymised by us (Van Nieukerken \& Jo- 
hansson 1987) on the basis of several non-type specimens which included S. suberivora and S. eberhardi. We choose the first to avoid change of names; the material does not include any S. ilicifoliella. Only the name Stigmella ilicivora ssp. nigra Dufrane has to be removed from synonymy with suberivora; below it is synonymised with $S$. ilicifoliella.

Material examined. - 980َ, 59 9 . - ALGERIA: 19 , Aurès, near Arris, $32 \mathrm{~km}$ SSE Batna, 1700m, 28.iv.1980, Q. rotundifolia, e.1. 7-9.vi.1980, E. J. van Nieukerken \& G. Bryan (ZMAN). - CROATIA: 1 \%, Zadar, 0-60m, 7.iii.1890, Q. ilex, e.1. 20.iv.1970, G. Deschka (SMNK). - FRANCE: $1 \hat{\sigma}$, Alpes Hte Provence: les Mées, 3-8.viii.1985, G.R. Langohr (JL); 1 ô, Alpes Maritimes: Cannes, Q. ilex, Constant, '9', '37' (as aureocapitella); 20 , Cannes, Q. ilex, Constant (as ilicivora) (MNHN); 3 ㅇ, Cannes, ii-iii.1887, Q. ilex, e.l. 9.v.1887, Walsingham (as 'type anypopta Joannis') (BMNH); 19 , Cannes, Saint Raphael, 7.iii.1890, Q. ilex, e.l. 20.iv.1890, Walsingham (BMNH); 10 , Cannes, Valescure, 07.iii.1890, Q. ilex, e.l. 03.v.1890, Walsingham (BMNH); 10 , Bouches-du-Rhône: 3 km W La Ciotat: La Grande Tête, Route des Crêtes, 11.ii.1991 Q. ilex, e.l. 21.iii.1991, E. J. van Nieukerken (RMNH); 2 , between Cassis and Cuges-les-Pins: Roquefort, 04.iii.1984, Q. ilex, e.l. 12-14.v.1984, R. Buvat (ZMAN); 10 , Charente: Angoulème, e.l. iv.1941, J. Sofner (RJ); 1 ㅇ, Hérault: St. Pons-de-Thomières, $2 \mathrm{~km}$ SE: Tombeilles, 20.ii.1991, Q. ilex, e.l. 5.vi.1991 E. J. van Nieukerken (RMNH); 10, 1 q , Var: Massif de l'Esterel, 20.vii.1979 G.R. Langohr (JL); 10 , Vaucluse: Pertuis, 10.vii.1991, A. \& Z. Laštùvka (AL); 1 q , Viens, 14.viii.1974, R. Buvat (MNHM). FRANCE, CORSICA: 10 , Castirla, $12 \mathrm{~km} \mathrm{~N}$ Corte, $400 \mathrm{~m}$, 20.vi.1994, B. Skule \& P. Skou (ZMUC). - GREAT BRITAIN: 20, 1 으, Dorset: Poole, 22.v.1941, W. Fassnidge (ZMAN); 10ิ, Poole, 17-20.v.1940, S.N.A. Jacobs (BMNH); 20 , 8 우, Gr London: Lambeth: Brockwell Park, 27.iii.1980, Q. ilex, e.l. 15-23.iv.1980, E. J. van Nieukerken \& A. M. Emmet (ZMAN, RMNH); 2 oे, 2 \% , London: Brockwell Park, Q. ilex, e.l. 15-18.iii.1975, A. M. Emmet (BMNH). - ITALY: 1 ô, Testico, 16.vii.1966, E. Jäckh; 40, Caserta: Baia Domizia, 25.vii.1972, R. Johansson; 8 ô, Baia Domizia, 7.viii.1972, R. Johansson; 1 ㅇ, Baia Domizia, 7.viii.1972, R. Johansson; 10 , Baia Domizia, 3.vii.1969, R. Johansson (; 1 \% , Latina: Monti Aurunci, 5 km N Itri, 600m, 24-30.vi.1969, R. Johansson; 20 , Monti Aurunci, 6 km N Itri, 600m, 15.viii.1972, R. Johansson (RJ); 1 \% , Savona: C. di Cadibona, 335m, 20.iv.1971, G. Baldizzone (GB); 1 , , Trieste: Sistiana Mare, 0-60m, 7.iii.1890, Q. ilex, e.l. 24.iv.1970 G. Deschka (SMNK). ITALY, SARDINIA: $7 \widehat{\delta}$, Bacu Trotu, Ortuabis, $800 \mathrm{~m}, 23 . v i i i$. 1978, 03.ix.1978, 23.vi.1979, G. Derra (GD, RMNH); 10 के, 8 ․ Mt. Istiddi, 700m, 19+28.v.1975, 30.vi.1976, 13.viii. 1977, 1.ix.1978, G. Derra (RMNH, GD); 40ิ, 2 ㅇ, Palolu, Meana-Sardo, 6.viii.1977, G. Derra (GD); 10 , Nuoro: 10 km W Dorgali, 26.vii.1999, P. Triberti (PT); 10 , Belvi, 650m, 20.vii.1984, J. H. Kuchlein (JHK); $1 \hat{\sigma}$, Belvi surroundings, 700m, 7.x.1975, F. Hartig (MRSN); 20 , Belvi, Mt. Istiddi, 900m, 9.vi.1977, F. Hartig (MRSN); 5 ô, 2 ㅇ, F. Cedrino, 5 km SW Galtelli, 16.vii.1999, P. Triberti (PT,RMNH); 40ે, 8 ㅇ, Mamoiada, 25.vii+30-31.vii.1983, J. H. Kuchlein (JHK, RMNH); 1 ㅇ, Seúi, 1100, 20.vii.1983, J. H. Kuchlein (JHK); 30 , 4으. Villanova-Strisaili, 1-15.viii.1983, 11-12+25.vii. 1984, J. H. Kuchlein (JHK). - ITALY, SICILY: $3 \hat{0}$, 1 오, Palermo: Cefalù, 500m, 9.vii.2000, P. Triberti (PT,RMNH). - SPAIN: 10 T. Cantabría: Picos de Europa: Camaleño, 520m, 25.vii.
1986, Mixed oak forest and meadows; at light ML or dusk,

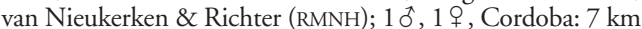
NE Santiago de la Espada, 1400m, 23.ix.1995, H.W. van der Wolf (HW); 10 , Murcia: 4 km NW Aledo, 26.ix.2001, C \& F K Gielis (RMNH); 1 ㅇ, Málaga, Ariate, $12 \mathrm{~km} \mathrm{E} \mathrm{Rhonda,}$ Casa Alta, 500m, 03.viii.1990, E. Traugott-Olsen (ZMUC); 20 , Málaga, Camino de Cesares, 500m, 9.vii.1973, E. Traugott-Olsen (ZMUC); 1 ô, Málaga, Camino de Istan, 200m, 08. vii.1973, E. Traugott-Olsen (ZMUC); 1 ô, Málaga, Camino de Ojen, 150m, 17.iv.1980, E. Traugott-Olsen (ZMUC); 1 to, Camino de Ronda, Urb. Madronal, Loma de Colmenas, 14.viii.1988 E. Traugott-Olsen (ZMUC); 7ో人, Camino de Ronda, Urb. Madronal, Loma de Colmenas, 500m, 3.ix. 1988, E. Traugott-Olsen (ZMUC, RMNH); 1 đo, Marbella, El Mirador, 14.iv.1971, E. Traugott-Olsen (ZMUC); 1 옹 Ronda, 29.iii.1979 Q, coccifera, e.l. v.1979, E. J. van Nieukerken (ZMAN); 1 o, Valencia: Gilet, 21.iv.1989, Q, coccifera, e.l. 15. v.1989, J. C. Koster (JCK). - TUNISIA: $10^{\top}, 7$ km S Ain Draham, les Chênes, 22.iii.1986, Q. suber, e.l., O. Karsholt (ZMUC); 60े, 4 ㅇ, Ain Draham area, 05.v.1988-18.v.1988, O. Karsholt (ZMUC, RMNH).

Additional unpublished records (A. \& Z. Laštüvka in litt.). - CROATIA: Dalmacia, Oračac; Istria, Rabac. - FRANCE: Bouches-du-Rhône, Aix en Provence; Vaucluse, Pertuis. ITALY; Catanzaro, Santa Caterina dell Iónio; La Spezia, Levanto. - ITALY, SICILY: Acate; Solarino. - PORTUGAL: Algarve, Lagoa da Nave, Salir (M. F. V. Corley in litt); Beira Baixa, Zebreira; Beira Alta: Serra da Estrella, Covilha; Serra da Estrella, Penhas de Saude.- SPAIN: Cádiz, Alcalá del valle; Castellon, Cuevas de Vinrona; Cuenca, Boniches; Cuenca, Gabaldón; Gerona, Anglés; Granada, Diezma; Granada, El Molinillo; Huelva, Bonares; Teruel, Albarracín; Teruel, Montalbán; Teruel, Segura de los Baños; Teruel, Vivel del Rio; Toledo, Robledo del Buey.

3. Stigmella ilicifoliella (Mendes) comb. n., stat. rev. (figs. 5, 46, 47, 51, 58, 92, 93, 128, 148, 166)

Nepticula sp.; Mendes 1913: 26.

Nepticula ilicifoliella Mendes, 1918: 127. Syntypes: SPAIN: Salamanca, Feb. 1912/1913 larvae on Quercus ilex, adults in May-June, Mendes (depository unknown) [not examined]. Nepticula ilicifoliella; Agenjo 1964: [unnumbered page 2 of appendix].

Stigmella ilicivora nigra Dufrane, 1955: 192. Holotype ô: PORTUGAL: e.l. 16.vi.1930, Quercus ilex [Mendes?] ex coll. Joannis, genitalia slide EvN3450 (KBIN) [examined], syn. n.

\section{Diagnosis}

Almost inseparable from the sympatric $S$. suberivo$r a$, except for the much narrower male genitalia, which can be seen without dissection with some experience. Males also have significantly longer antennae than suberivora, but there is some overlap, in females the difference is less obvious. Valvae not bulging, without field of papillae, but with a field of many setae, genitalia capsule longer than wide. Female genitalia with ductus spermathecae with 4.5 convolutions, ratio bursa /accessory sac 2.3-3, smaller than in suberivora; usually more setae on T8. 


\section{Description}

Male (fig. 5). - Forewing length 2.5-3.0 mm, wingspan $6.0-6.6 \mathrm{~mm}$. Head: frontal tuft from almost white or ochreous to yellowish orange, occasionally black, collar pale grey to dark grey-fuscous. Scape pale yellow. Antenna slightly darker, $3 / 5$ of forewing length, with 39-50 segments, ratio to forewing length 15-19 segments $/ \mathrm{mm}$. Thorax and forewing unicolorous dark bronze brown, at base and on thorax almost bluish black in fresh specimens, a faint purplish lustre at apex; terminal cilia concolorous, underside bronzebrown. Hindwing coarsely scaled, dark grey-brown, dorsum with long dark brown to bluish black androconial scales, extending into fringe, the longest near base, approximately $3 / 4$ of cilia length; along basal half of costa long curved, spatulate bluish-black androconial scales, the longest as long as or longer than the shortest dorsal androconial scales; underside grey brown, cilia dark grey. Abdomen dark grey brown, with conspicuous white to pale yellowish anal tufts inserted on well sclerotized plates; genital capsule not particularly broad, valvae with dense tuft of hairs.

Female. - Forewing length 2.5-2.9 mm. Antennae with 33-40 segments, ratio to forewing length 12-16 segments $/ \mathrm{mm}$. As male, but collar yellowish white to pale yellow, hindwing grey, androconial scales absent, abdomen dark grey-brown, without anal tufts.

Male genitalia (figs. 46, 47, 51, 92, 93). - Measurements: see table 3. Capsule longer than wide or almost as long as wide. Vinculum anteriorly slightly bilobed. Uncus with widely separated horns. Gnathos with widely separated posterior processes with club-shaped tips. Valva with pointed distal process less than $1 / 4$ valva length, pointing slightly inwards(fig. 46, 51); inner margin forming a rounded lobe, ventral outer lobe pronounced, but not as bulging as in suberivora, covered with field of dense setae, but no papillae (fig. 46); sublateral processes short. Aedeagus with vesica with a more or less curved group of cornuti in basal half; this band comprises about 20-30 large cornuti with serrated bases and many small spinelike cornuti; more distally there is a group of ca 7-12 long needle-shaped cornuti and a few near phallotrema; manica covering aedeagus almost completely, except anteriormost part, covered with small spines and pectinations.

Female genitalia (figs. 58, 128, 148). - T8 with ca $25-50$ setae. Bursa length $1062-1204 \mu \mathrm{m}, 2.3-3.0 \times$ as long as accessory sac. Bursa distally covered with minute pectinations, difficult to see (observed with Differential Interference Contrast only); accessory sac anteriorly with pectinations only. Ductus spermathecae with $4.5(\mathrm{n}=3)$ convolutions.

\footnotetext{
Biology

Hostplants. - Quercus ilex**, Q. rotundifolia**, Q. suber**.
}

Leafmine. - The few mines studied are similar to suberivora, but all almost invisible because they are covered by an uneaten part of the leaf parenchyma; this was also described by Mendes (1913, 1918). It is not yet clear if this character will always separate ilicifoliella from suberivora, or whether it depends on the rather thick leaves of $Q$. rotundifolia in which we (and Mendes) found the mines.

Life-history. - Larvae have been collected in February and March. Adults have been collected from June to early September, voltinism unknown.

\section{Distribution (fig. 166)}

Widespread in Portugal and Spain, particularly inland, but probably less common than suberivora. In France (new record) known from old specimens along the Atlantic coast near Bordeaux and in the Hérault and the Côte d'Azur near Cannes; to be expected along the Italian Riviera and in North Africa. Often sympatric with $S$. suberivora. Frequently found in mountains; recorded from sea level up to $2200 \mathrm{~m}$ in the Sierra Nevada.

\section{Remarks}

Although several earlier authors considered more than one species of this group to be feeding on evergreen oaks, in the last decades only one species was recognized and other names were considered to be synonyms (Johansson 1971). The names ilicivora and aureocapitella are treated above, but other authors still considered the possibility of more species. Joannis (1909) wondered whether specimens with dark abdomen might belong to a different species, but still kept them in Nepticula ilicivora Peyerimhoff. Constant also considered material with larger mines on Quercus suber as a different species. Such material, belonging to the species under discussion, is present in the Lhomme and Ragonot collections in Paris and labelled with 'suber gr. m.' and 'sp. n. Cst.' (plus a pink triangle, which stands for coastal area of Alpes Maritimes, near Cannes, see Boursin 1936). We think that gr. m. stands for 'grande mine'. We have not been able to find any paper by Constant with this information, but a manuscript name suggested by him (suberoidella) has still entered the literature (Le Marchand 1946, Skala 1938), although the source of this remains unknown.

We consider that this species, which we only discovered when working on this paper, should be named ilicifoliella Mendes. After its original description, this name has only once been cited, in a checklist (Agenjo 1964). It is unclear why everybody, including ourselves, have overlooked this name. Possibly it was - incorrectly - considered a misspelling of $N$. ilicis Mendes.

The identity of ilicifoliella is based on the following facts: 


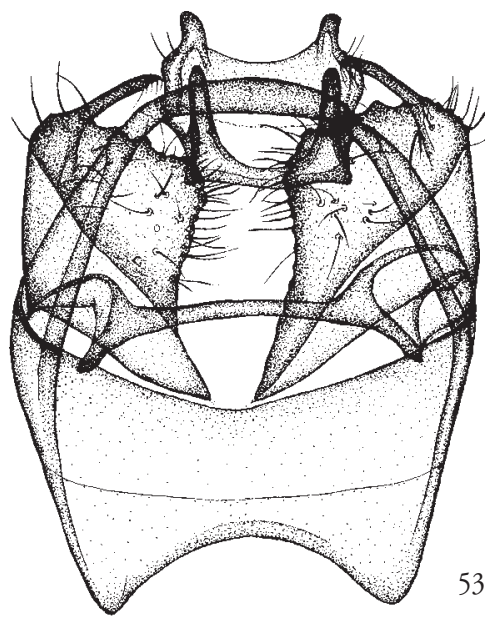

53

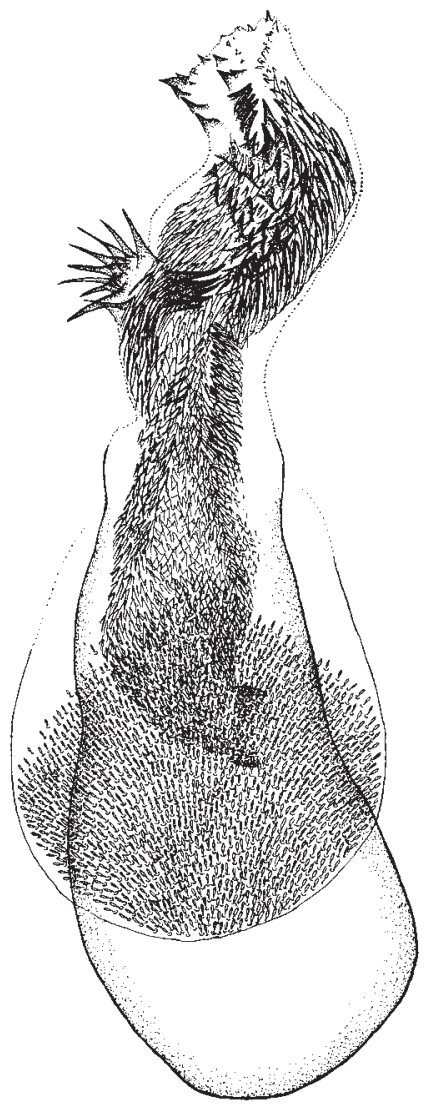

54

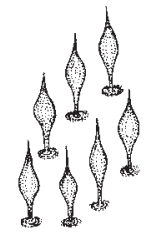

55

Figs. 53-55. Stigmella kasyi, male genitalia, holotype, slide RJ1528. - 53, Capsule; 54, aedeagus, vesica partly everted; 55 , detail of spines on manica.
1. Mendes (1913) described an unknown species (Nepticula sp.) from Quercus ilex in São Fiel (Portugal), with a dark abdomen (darker than typical 'ilicivora Peyer.'), and with almost invisible mines, only visible when held against the light. He believes it likely to be a new species ('Provavelmente é uma especie inedita' ).

2. Mendes (1918) described Nepticula ilicifoliella n. sp. from Salamanca very briefly, translated as follows:

'I will describe it briefly. End of May and early June. Caterpillar: on Holm-oak (Q. ilex) during the whole month of February. One can discover the mine by the prominence on the underside of the leaf.'

Although he does not refer to his earlier paper, he almost certainly was naming here the unknown species from Quercus ilex. This is indicated by the mine character, and the fact that all the other species known to him from this oak, were also mentioned in both papers. Despite the fact that the brief description is based on the mine, the name is available, since descriptions based on 'the work of an animal' published before 1930 are available (ICZN, article 1.2.1, 13.3.3).

3. Dufrane (1955) mentioned a male 'Stigmella ilicivora with a dark abdomen from Portugal, which he received from J. de Joannis. Dufrane described this specimen as subspecies nigra of S. ilicivora.

4. Mendes had frequent contact with Joannis, and sent him material regularly (Luisier 1944, Zerkowitz 1946). There are remnants of the original collection of Mendes in the monastery of Sante Tirso and in the high school of Castelo Branco (partly destroyed), but mostly without name labels and probably without the types (E. Maravalhas in litt., September 2003). Most likely the types of Nepticula ilicifoliella must therefore be considered lost. Up to now, Joannis material has served several times as type material of Mendes' Nepticulidae (e. g. Nepticula ladaniphila Mendes, 1910, see Van Nieukerken 1983). In the Joannis collection there are five specimens labelled 'Q. ilex Portugal 4 juillet' which we think were sent to him by Mendes. Probably Mendes and Joannis discussed the possibility of a second species on Quercus ilex. These specimens are conspecific with the holotype of nigra, which most likely also belongs to the material Joannis got from Mendes. The year 1930, given by Dufrane, may be a mistake, and it is not cited on the original label in Joannis' handwriting, as is the usual case in Joannis material; 1930 may have been the year Dufrane received the specimen. Mendes was expelled from Portugal as Jesuit, and lived in Spain afterwards and finally in Brasil (Luisier 1944, Zerkowitz 1946). He therefore could not have collected a Portuguese specimen after 1910, unless it was presented to him by someone else (when living in Spain, he lived some time near the border with Portugal). The Portuguese material serves for establishing the identity, but cannot be regarded as types, 
because Nepticula ilicifoliella was clearly described from Spain (Salamanca).

On the evidence presented here, we consider Stigmella ilicifoliella (Mendes) to be the valid name for this species, with which we synonymise $S$. nigra Dufrane.

We have not been able to confirm the character 'dark abdomen', mentioned by Mendes, Joannis and Dufrane. Both S. suberivora and ilicifoliella are somewhat variable in this respect, and fresh specimens usually have much darker abdomens than faded collection material.

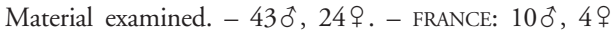
[pink triangle=Alpes Maritimes: Cannes] 'suber, gr. m.' [Quercus suber, Grande mine], decades 16-20 [1.vi-15.vii, 19th Century] [Constant], colls. Ragonot and Lhomme, partly labelled as 'sp. n. Constant' (MNHN); 10 , Gironde, Pessac, e.l. 17.v.1929, Quercus ilex, Le Marchand (MNHN); 10े, Hérault, St. Pons, ix.1904, Chrétien (MNHN). - SPAIN: $3 \hat{\sigma}$, Albacete, Biencervida, 1000m, 16.vi.1986, P. Skou (RJ); 1 ơ, Barcelona, La Llacuna, Anoia, 28.viii.2000, E. Requena (RMNH); 1, Barcelona, San Marti de Tous, Anoia, 29. vii.2000, E. Requena (RMNH); $20 \hat{\text {, }} 2$ 2 , Burgos, Ibeas de Juarros, 884m, 23.vii.1985, A. Vives Moreno (AV, RMNH); $2 \widehat{0}$, 1 , Cantabría, Picos de Europa: Camaleño, 520m, 25.vii. 1986, van Nieukerken \& Richter (RMNH); 10 , Cuenca, Cuenca, 900m, 16.vii. 1986, C. Gielis (CG); 2 đิ , Granáda, 20 $\mathrm{km}$ ENE of Granada, 23.vii.1986, C. Gielis (RMNH, CG); 1 , Granáda, N 342, Rio Baza, 7-9.vii.1985, G. Baldizzone \& E. Traugott-Olsen (ZMUC); 19 , Granáda, Puerto de Mora, 22.vii.1986, C. Gielis (CG); 90 , 11 , , Madrid, Cadalso de los Vidrios, $2 \mathrm{~km}$ W, 7.viii.1986, van Nieukerken \& Richter (RMNH); 20 , Madrid, Lozoya, 3 km E., 1100m, 02.iii.1997, leafmines, Quercus rotundifolia, e.l. 11.v.1997-16.v.1997, E. J. van Nieukerken (RMNH); 1 9, Málaga, 4 km NE Igualeja, Serrania de Ronda, 1100m, 7.ii.1984, leafmines, Quercus rotundifolia, e.l. 8.iii.1984, E. J. van Nieukerken (RMNH); 20 , Málaga, Camino de Istán, 28.vi.1972, 8.vii.1973, E. Traugott-Olsen (ZMUC); 10 , Murcia, Sierra d'Espuña, C.F. de las Alquerias, 800m, 04.iv.1997, H. W. van der Wolf (coll. van der Wolf); $1 \delta^{\star}$, Teruel, Noguera, 1500m, 11.vii.1986, C. Gielis; 10 , Teruel, Sierra Alta, 10.vii.1985, C. Gielis (CG). PORTUGAL: 10 , holotype of nigra; $3 \delta^{\star}, 2$, [Beira Baixa: Sao Fiel], 4.vii, Quercus rotundifolia [as ilex], [Mendes], coll. Joannis (MNHN); 10, Covao do Boi, Serra da Estrela, 1870m, 4.ix.2001, M. F. V. Corley (MC).

Additional records. - PORTUGAL: 10 , Algarve, N. of Alportel, 6.ix.1991 M. F. V. Corley (in litt.); 1 đิ (black-headed), Algarve, Messines de Baixo, 25.vi.2002; 1 ㅇ, Beira Alta, Covilha, 21.vi.2002; 30 , Sétubal, Marateca, 100m, 24.vi.2002. - SPAIN: 2 ơ, Cadiz, Alcala del Valle, 11.vii.1993; 1 đิ, Granáda, Diezma, 29.vi.2003; 3 ô, Granáda, El Molinillo, 28.vi.1992; 20 , Granáda, Sierra de Guillimona, 29.vi. 2001, 10 , Granáda, Sierra Nevada, Puerto de la Ragua, 2200 m, 8.vii.1993; 1 ô, Málaga, Jimena de Líbar, 500m, 27.vi.2003 (all A. \& Z. Laštüvka in litt.).

\section{Stigmella cocciferae sp. n.}

(figs. 6, 7, 39, 48, 49, 52, 59, 94, 95, 129, 149, 166)

Nepticula sp.; Amsel \& Hering 1931: 141 [records of mines on Quercus coccifera].
Type material. - Holotype ô: GREECE, Arákhova (Voiotía), cult. terraces, olive \& almond, $950 \mathrm{~m}$, 38.29N-22.35E, 27-29.ix.1980, at light, S. B. J. Menken \& E. J. van Nieukerken. genitalia slide VU 0813 (RMNH).

Paratypes: $33 \hat{0}, 10$. - GREECE (mainland): $1 \hat{0}$, 49 , same data as Holotype (RMNH); 29 , Akhaia, Kalávrita, 20.vi.1997, A. Laštúvka (AL); 10 , Argolis: Akhladokambos, $18 \mathrm{~km}$ E of Tripolis, 5.x.1982, Gg. Derra (GD); 20 , Ioánnina: Asprángeli, 10.vi.1997,

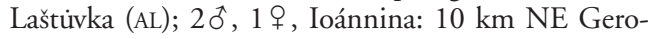
platanos by Konitsa, 800, 24.vii.1990, M. Fibiger; 10, Ioánnina, 4 km SW Geroplatanos, 800, 12.vii. 1998, D. Nilsson \& B. Skule; 1 đ, Lakonia: 5 km S Monemvasia, 16.v.1987, G. Christensen (ZMUC); 10 , Lakonia: Taygetos, 1000-1200 m, 12.vi.1979, MV, Gozmány \& Christensen (ТМAB); 10 , Messinia: Kardhamíli, gorge with maquis, 18. ii.1990, Quercus coccifera, e.l. 21.iii.1990, E. J. van Nieukerken; 1 옹 Voiotia: Parnassos Oros, Arakhova surr., $800 \mathrm{~m}$, 28.ix.1993, Lingenhöhle (RMNH). - GREECE, KOS: 4 đ, 1 ․ Kos, Asfendiou, 5-12.x.1988, R. Johansson (RJ, ZMUC); 10 , Kos, 5 km E of town Kos, 1.x.1988, R. Johansson (RJ). - GREECE, KRITI: 10 , Iraklion: Potamies monastery, 19.iv.1989, R. Johansson (RJ). GREECE, SAMOS: $1 \%$, I. Samos, Kokkari, $10 \mathrm{~m}$, 22.vi.1996, R. Sutter (RS). - ISRAEL: 10 , Kirjat-Anawim (Qiryat Anawim), Jerusalem, 2.v. 1930, H. G. Amsel (LNKD). - TURKEY: 10 , Ankara: $20 \mathrm{~km} \mathrm{NW}$ Kizilcahamam, 1200 m, 24.vii.1986, M. Fibiger (RJ); 60ે, Antalya: Antalya 20 km W, 28.iv. 1996, K. Nupponen \& J. Junnilainen (RMNH, JJ); $1 \hat{\sigma}$, Antalya: Bucak, $35 \mathrm{~km} \mathrm{S,} \mathrm{850m,} \mathrm{14.v.2000,} \mathrm{J.} \mathrm{Junnilainen} \mathrm{(JJ);}$ 10, Içel [Mersin]: $5 \mathrm{~km}$ NW Erdemli, 200m, 16.vii.1986, M. Fibiger, (ZMUC); 10 , Içel (Mersin): Güzeloluk, NW Erdemli, Taurus, $1400 \mathrm{~m}$, 16.vii.1986, M. Fibiger (RJ); 40 , Isparta: Isparta, 20 $\mathrm{km}$ SE, 14.v.2000, J. Junnilainen (RMNH, JJ); 10 , Izmir: $30 \mathrm{~km}$ NW Bergama, 500-750m, 10-12. v.1993, O. Karsholt (ZMUC); 10, Konya: 20 km SE Hadim, Taurus, 1800 m, 14.vii.1986, M. Fibiger (RJ).

Additional records (A. \& Z . Laštuivka in litt.). - GREECE:

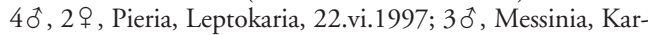
damili, 19.vi.1997; 60ે, Lakonia, Apidia, 15.vi.1997; 20 , Voiotia, Khairona, 13.vi.1998. - TURKEY-IN-EUROPE: Kemerburgaz, el. 25.iv.1975, G. Deschka (AL).

\section{Diagnosis}

Very similar to Western European S. ilicifoliella. The latter usually is darker and has the androconial scales extending to tip of hindwings, whereas cocciferae has no androconial scales in distal $1 / 4$; further androconials in S. cocciferae paler and shorter than in ilicifoliella. Male genitalia: in S. ilicifoliella the capsule and valvae are markedly wider than in cocciferae and gnathos horns are club-shaped; in S. ilicifoliella the manica 


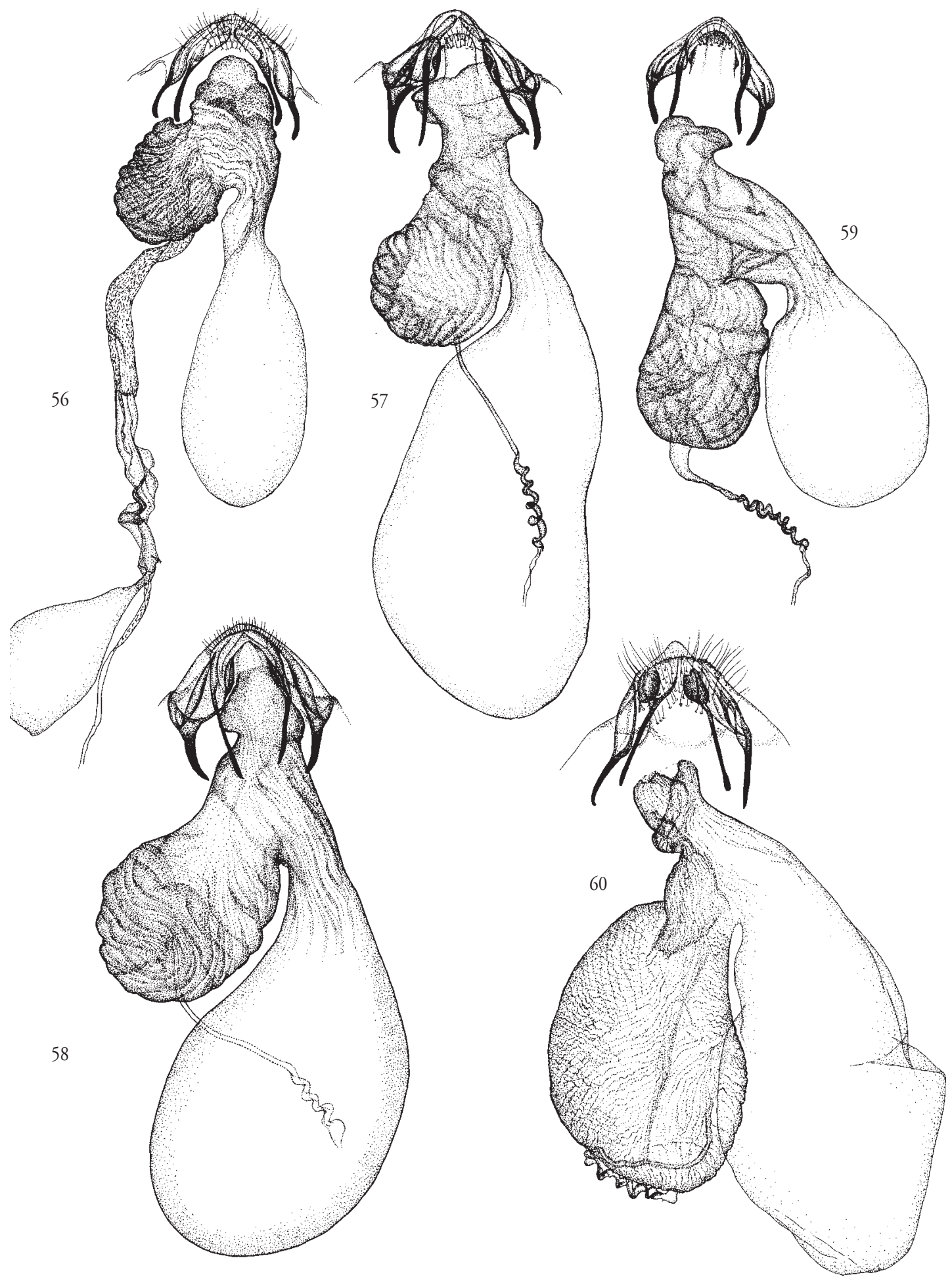


covers the aedeagus almost completely and in cocciferae only distal $2 / 3$. Female genitalia very similar to $S$. suberivora and ilicifoliella, but bursa in suberivora and ilicifoliella much longer compared to accessory sac than in cocciferae.

\section{Description}

Male (fig. 6). - Forewing length 2.2-3.2 mm, wingspan 4.9-7.0 mm. Head: frontal tuft yellowish orange, collar fuscous. Scape pale yellow. Antenna dark bronze brown, $3 / 4$ of forewing length, with $36-49$ segments, ratio to forewing length 13-19 segments $/ \mathrm{mm}$. Thorax and forewing unicolorous dark bronze brown, weakly shining; terminal cilia concolorous, slightly paler at tip; underside bronze-brown. Hindwing coarsely scaled, fuscous, distal $1 / 4$ brown, basal $3 / 4$ of dorsum with long dark grey-brown androconial scales, extending into fringe, the longest near base, approximately $2 / 3$ of cilia length; along basal half of costa long curved, spatulate bluish-black androconial scales, the longest as long as or longer than the shortest dorsal androconial scales; underside grey brown, cilia dark grey. Abdomen dark grey brown, with pale yellowish anal tufts inserted on well sclerotized plates.

Female (fig. 7). - Forewing length 2.2-2.9 mm, wingspan 4.9-6.2 mm. Antenna with 30-37 segments, ratio to forewing length $12-15$ segments $/ \mathrm{mm}$. As male, but collar pale yellow, hindwing grey, androconial scales absent, abdomen dark grey, without anal tufts.

Male genitalia (figs. 48, 49, 52, 94, 95). - Measurements: see table 3 . Capsule relatively narrow compared to related species. Vinculum bilobed anteriorly. Uncus with widely separated horns. Gnathos with long, widely separated posterior processes. Valva with pointed distal process less than $1 / 4$ valva length, in lateral view medial (fig. 52); inner margin almost straight with almost rectangular distal angle; sublateral processes about $1 / 3$ transtilla length. Aedeagus with vesica with a more or less curved group of cornuti in basal half; this band comprises about 35-40 large cornuti with serrated bases and many small spinelike cornuti; more distally there is a group of ca 12 long needle-shaped cornuti and one larger near phallotrema; manica covering distal $2 / 3$ of aedeagus, covered with small spines and pectinations.

Female genitalia (figs. 59, 129, 149). - T8 with more than 20 setae. Bursa well visible, total length $1050-1120 \mu \mathrm{m}, 2.4-2.6 \times$ as long as accessory sac; walls thin, without pectinations. Accessory sac more strongly sclerotized, without pectinations. Ductus spermathecae with ca 6-7 convolutions.

\section{Biology}

Hostplant. - Quercus cocciferd ${ }^{* *}$. It was twice reared from this host and in Greece otherwise always collected near $Q$. coccifera, often with vacated mines.

Leafmine (fig. 35). - A broad gallery with broad frass, egg on leaf upperside. Similar to that of $S$. suberivora.

Life-history. - Probably bivoltine, or even with more generations. Larva collected in February, adults flying in April-July and September-October.

\section{Distribution (fig. 166)}

Widespread in Eastern Mediterranean: mainland Greece, Aegean islands, Crete, Turkey and Israel. Occurs from sea level up to $1800 \mathrm{~m}$ in Taurus. To be expected in Cyprus, Syria and Lebanon. It seems to occur completely vicariantly with $S$. suberivora, of which the closest records are in south Croatia (Zadar). No records of either species are known between northern Greece and Zadar.

\section{Etymology}

A noun in genitive case: named after the foodplant Quercus coccifera.

\section{Stigmella kasyi sp. $\mathrm{n}$.}

(figs. 8, 9, 53-55, 60, 96, 130, 150, 167)

Type material. - Holotype ô: AFGHANISTAN, 25 km. N Barikot, Nuristan, 1800 m, 12-17.vii.1963, Kasy \& Vartian, genitalia slide RJ 1528 (NHMW).

Material excluded from type series. $-1 q$, AFGHANISTAN: Paghman, 30 km NW Kabul, 2500 m, 20+24.vii.1965, Kasy \& Vartian (NHMW).

\section{Diagnosis}

Male externally resembling other species with spatulate androconial scales, but much larger, larger number of antennal segments than any species in this group. Otherwise, the black head and edged scape and paler forewings separate it from S. suberivora, ilicifoliella and cocciferae. The black-headed S. atricapitella and ruficapitella have no edged scape. Female very similar to all other red-headed species, except for large size and large number of antennal segments. Male genitalia characterized by numerous cornuti and very specially shaped spines on manica Female genitalia recognized by large size of accessory sac (bursa less than $1.5 \times$ as long as accessory sac) and number of convolutions.

Figs. 56-60. Stigmella spp., female genitalia. - 56, S. fasciata, holotype, slide EvN/VU 1964; 57, S. suberivora, Tunisia, slide RJ 1667; 58, S. ilicifoliella, Spain, slide RJ2275A; 59, S. cocciferae, Greece, Kos, slide RJ1726; 60, S. kasyi, Afghanistan, slide Mus. Vind. 5408. 


\section{Description}

Male (fig. 8). - Forewing length $3.45 \mathrm{~mm}$. Wingspan $7.5 \mathrm{~mm}$. Head: frontal tuft dark grey brown, collar grey-brown. Scape white, with broad distal bluish grey edge, almost half scape width. Antenna grey brown, $3 / 4$ of forewing length, with 53 segments. Thorax and forewing unicolorous pale bronze brown, slightly darker at basal $1 / 4$ of costa; terminal cilia slightly paler; underside pale grey-brown, cilia pale grey; at second $1 / 3$ of dorsum a large brush of brown androconial hair-scales, arising from edge, approximately as long as cilia. Hindwing pale yellowish grey, grey-brown androconial scales on dorsum extending into fringe, approximately $2 / 3$ of cilia length; along basal third of costa with broad grey-brown androconial scales, the longest as long as hindwing width; underside pale grey. Abdomen removed for dissection (reconstructed in fig. 8). Anal tufts inserted on well sclerotized plates.

Female (fig. 9). - Forewing length $3.8 \mathrm{~mm}$. Wingspan $8.6 \mathrm{~mm}$. Frontal tuft yellowish brown; collar pale yellowish. Scape pale yellowish, with some pale grey-brown scales. Antenna with 48 segments. Otherwise as in male, except for secondary sexual characters. Abdomen removed for dissection.

Male genitalia (figs. 53-55, 96). - Measurements: see table 3. Vinculum anteriorly bilobed. Uncus with widely separated horns. Gnathos with widely separated posterior processes. Valva with thin and long pointed distal process; inner margin slightly concave with almost rectangular distal corner; sublateral processes about $1 / 3$ transtilla length. Aedeagus very long; vesica with numerous small and needle-shaped cornuti; a group of longer needle-shaped cornuti visible at left side (the vesica is somewhat everted in holotype, position therefore different compared to in situ slides); manica not very large, ventrally covered with a special type of papillate spines.

Female genitalia (figs. 60, 130, 150). - T8 not well visible in single slide, probably with longitudinal depressions. Bursa well visible, total length $1250 \mu \mathrm{m}$, $1.5 \times$ as long as accessory sac; walls thin, without pectinations. Accessory sac more strongly sclerotized, without pectinations. Ductus spermathecae with 6 convolutions.

\section{Biology}

The male was taken in July in extensive forests of Quercus baloot, which we presume to be the hostplant (cf. Kasy 1965). Q. baloot belongs to the species group of $Q$. ilex.

\footnotetext{
Distribution (fig. 167)

Only known from eastern Afghanistan.
}

\section{Remarks}

The female is believed to belong to the same species on the following grounds: it agrees very well in external features, in particular in the large size (one of the largest known Stigmella species) and the colour of the forewings. Further both male and female show a close relationship to S. suberivora, ilicifoliella and cocciferae, and are currently the only specimens of this species group known from Afghanistan. However, the locality Paghman on the label of the female is quite different from the type-locality; Paghman is a dry mountain northwest of Kabul, and no Quercus is noted in the list of plants of that locality (Kasy 1967). Three Quercus species (Q. baloot, Q. semecarpifolia Smith and Q. dilatata Royle) occur in Afghanistan (Menitskij 1984), all confined to the wetter extreme east of the country, some large valleys and southern slopes of the Hindu Kush mountain ranges, relatively far away from Kabul (see fig. 167).

This specimen was collected in 1965, on an expedition which also included collecting in the Quercus forests of Nuristan (Kasy 1967). It is therefore possible that the female was accidentally mislabelled, but it is also possible that planted oaks occur closer to Paghman or that the female was a vagrant. Since the possibility exists that the female is not conspecific with the male after all, we have excluded it from the type-series.

\section{Etymology}

A noun in genitive case. It is an honour to dedicate this species to the late Dr. Friedrich Kasy (1920-1990, see Fischer 1991), to whom we owe much for his careful collecting of many Nepticulidae both in the Middle East and in Austria, and his kind support of our work.

\section{Stigmella basiguttella (Heinemann)}

(figs. 10, 11, 97, 98, 131, 151, 168)

Nepticula basiguttella Heinemann, 1862: 258. Lectotype $q$ : GERMANY: Br[aun]schw[eig], Eichen e.l. [Quercus], Heinemann, Genitalia slide on pin (LMHG) [examined].

Stigmella cerricolella Klimesch, 1946: 160. Syntypes: ITALY: Ferrania (Ligur. Appenin), xi.1944, larvae on Quercus cerris, e.l. 20.iv-5.v.1945, Klimesch (ZSMG) ['paratype' examined].

Nepticula basiguttella; Petersen 1930: 46, fig. 9 (male genitalia), Johansson 1971: 253, Borkowski 1972: 779, Klimesch 1978: 243.

Nepticula basiguttella cerricolella; Johansson 1971: 253, Borkowski 1972: 781.

Stigmella basiguttella; Beirne 1945: 198, fig. 14 (male genitalia), Emmet 1976a: 244, Johansson \& Nielsen 1990: 229, Puplesis 1994: 158, A. \& Z. Laštủvka 1997: 105.

\section{Diagnosis}

One of the most variable species of the group, but always with dark head. The amount of pale patches on 
the forewings varies, but usually the basal spot is present and frequently the middle part of the wing is slightly paler to almost white. Similar species are, in the males, S. trojana and bicuspidata, which have narrow spatulate androconials along hindwing costa, $S$. macrolepidella, which usually is much paler, including a pale abdomen and $S$. samiatella, which has uniform forewings and brown hindwings. Females may in addition resemble those of $S$. atricapitella and S. zangherii.

Male genitalia (measurements see table 3) characterized by the extremely long and straight aedeagus with some large cornuti at phallotrema, and absence of manica. Female genitalia by the characteristic arrangement of spines in the accessory sac.

\section{Biology}

Hostplants. - Castanea sativa*, Quercus castaneifolia (Laštüvka \& Huemer 2002), Q. cerris**, Q. frainetto (Buhr 1940), Q. ithaburensis subsp. macrolepis (Klimesch 1978), Q. petraea**, Q. pubescens**, Q. pyrenaica* (Mendes 1913), Q. robur*, Q. rubra*. The record on $Q$. castaneifolia is based on light-collected specimens in forests with only this oak present.

Leafmine. - Very characteristic gallery, completely filled with neatly contorted green frass, becoming brown after the larva has left the mine. Egg invariably on upperside of leaf, usually close to or on a vein. Larva bright green, described in detail by Gustafsson \& Van Nieukerken (1990). This is the only widespread species that can always be recognised by mines and larvae, thus also vacated mines give reliable records.

Life-history. - Bivoltine, or possibly with more generations in Mediterranean. Larvae found from June to November, adults May-early September.

\section{Distribution (fig. 168)}

The most widespread species in the group, almost following the distribution of Quercus: throughout Europe, North Africa and Southwest Asia up to northern Iran (Laštu̇vka \& Huemer 2002, and one specimen cited below). No positive records yet from Albania, Belarus, Bosnia, Ireland or Latvia. New records from Azerbaijan, Georgia and Tunisia. Highest altitude: 1900 m (Sierra Nevada).

\section{Remarks}

In their analyses of allozymes, Cronau \& Menken (1990) found the presence of two isolated forms in three Dutch populations of basiguttella, which they considered to be two different, cryptic species. Up to now, we have not seen any other evidence of two distinct species, despite the great variability in colour pattern. Further research on these populations is needed, preferably using DNA markers in addition to detailed morphological research.
Material examined. - 740, 33,+ 4 adults, various leafmines. - AUSTRIA: 1 ․ - AZERBAIJAN: $2 \hat{0}, 20 \mathrm{~km}$ E Lerik, 4.vii.1964, A. Zagulaev; 60, 5ㅇ, Lenkoran, env., 7.viii. 1987, Ivinskis (ZIN). - BELGIUM: 10 , 7 \% . - CROATIA: 10 , Krk, Kampelje, 17.viii.2001, G. Baldizzone; 2 क , Krk, Str. Krk-Vrbnik, 23.vii.1988+30.vii.1990, G. Baldizzone (GB); 1 đo, Krk, Misucaynica, 17.vi.1976, E. Jäckh; 2 đે, Krk, Vrbnik, 19+27.vi.1976, E. Jäckh (USNM); $3 \hat{0}$, Velebit, 17 km E Karlobag, 26.vi.1983, B.Å. Bengtsson (B̊̊B). - FRANCE: 20 , 2 adults. - GEORGIA: 40े, Lagodekhi, 13-14.vii.1986, S. Seksyaeva \& S. Sinev; 4ô, ibid., 30.viii.1986, M. Kozlov (ZIN). - GERMANY: $1 \hat{0}, 1$ ㅇ. - GREECE: 1 옹 Ioánnina, $10 \mathrm{~km}$ NE Geroplatanos by Konitsa, 800m, 24.vii.1990, M. Fibiger; 10े, Larissa, Olympos, 700-2100m, 21-26.v.1990, O. Karsholt (ZMUC); leafmines: Evritanía, $4 \mathrm{~km} \mathrm{~W} \mathrm{Palaiokas-}$ tron, 21.ix.1980, Quercus petraea, Menken \& van Nieukerken; Evvoia, Dhírfis Oros, 11+12.ix.1980, Castanea sativa + Quercus pubescens, Menken \& van Nieukerken; Fthiotis, 1-2 $\mathrm{km}$ W Timfristós village, 20.ix.1980, Castanea sativa, Menken \& van Nieukerken (RMNH, ZMAN). - HUNGARY: 1 ㅇ. - IRAN: 10, $79 \mathrm{~km}$ E Azad Shahr (as Shah-Passand),

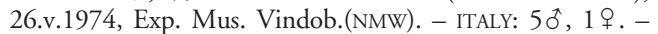
NETHERLANDS: $17 \widehat{\delta}, 7 q, 2$ adults. - PORTUGAL: 19 , Beira Alta, Caldas de Manteigas, 850m, 4.ix.2001, M. F. V. Corley (MC); Leafmines, Tras-os-Montes, PN Montesinho, 8 km N Vinhais, 27-30.vii.2001, Quercus pyrenaica, E.J. van Nieukerken (RMNH). - POLAND: 1 ․ - RUSSIA: $2 \hat{0}$, Borisovka, Les na Vorskle res., at light, 27.vi+ 19.viii.1985, Krivochatskij (ZIN); Leafmines, Kalmykia, $160 \mathrm{~km}$ W Elista, $20 \mathrm{~km} \mathrm{SW}$ Yashalta, 25.ix.2000, Quercus robur, V. Zolotuhin (vZ). SLOVAKIA: 20 . - SPAIN: 10 , Granada, Cherin, 26.v. 1999, J. Junnilainen (JJ); $1 \hat{\delta}$, Granáda, Sierra Nevada, Ruta de Veleta, 1900m, 21.viii.1984, M. Kavin \& P. Skou; 1ô, Málaga, Camino de Ronda, Urb. Madronal, Loma de Colmenas, 3.ix.1988, E. Traugott-Olsen (ZMUC); 40ิ, 1 ㅇ, Salamanca, San Miguel de Valero N, $3 \mathrm{~km}$ S of Linares de Riofrio, 850m, at light 2.viii.1986, van Nieukerken \& Richter (RMNH); 10, Segovia, Riazza, 3.viii.1986, C. Gielis; 10 , Teruel, Noguera, 1500m, 11.vii.1986, C. Gielis (CG). - SWEDEN: 3 ô, 3 \%. -

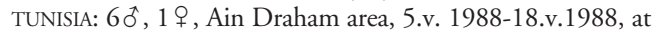
light, O. Karsholt (ZMUC, RMNH). - TURKEY: Adana, Taurus, 4 km NE Feke, 1000m, 7.ix.1983, G. Derra (GD).

Additional records (A. \& Z. Laštùvka, in litt.). - GREECE: 10ิ, Ioannina, Kepessovo, 13.vi.1996; $1 \hat{\sigma}$, Kastoria, Aposkepos, 11.vi.1996. - MACEDONIA: Trojáci; Istibani. - SPAIN: 3 đo, Cáceres, Piornal, 1200m, 20.vi.2003; 3 ô, Cuenca, Boniches, 1200m, 17.vi.2002; 20 , 2 우, Teruel, Montalbán, 1000m, 16.vi.2002; 15.vi.2003; 30 , Toledo, Robledo del Buey, 800m, 18.vi.2002.

\section{Stigmella macrolepidella (Klimesch)}

(figs. 12, 13, 61-63, 77, 99, 132, 152, 169)

Nepticula macrolepidella Klimesch, 1978: 257. Holotype ô: GREECE: Rhodos, Rodini, Mine on Quercus macrolepis, 22.ix.1972, Zucht Nr. 1054, e.l. 15.x.1972, Klimesch (ZSMG) [paratypes examined].

Stigmella macrolepidella; Van Nieukerken 1986b: 13, Z. \& A. Laštüvka1998: 318.

[Nepticula ruficapitella; Amsel \& Hering 1931: 140 (empty mines on $Q$. ithaburensis ssp. ithaburensis, misidentification, most likely this species)]. 


\section{Diagnosis}

The palest and one of the smallest species, pale pattern usually predominant, also abdomen almost completely white. Male can only be confused with specimens of $S$. trojana or basiguttella with extensive pale pattern. The darker females can also be confused with S. zangherii or dorsiguttella (the latter are pale-headed!), but they have a darker abdomen and usually a darker wing pattern; the ovipositor of macrolepidella is also pointed. Male genitalia very characteristic with the long cornuti anteriorly, the bilobed uncus processes and shape of valva. Female genitalia with spines in posterior half of accessory sac, rather similar to $S$. szoecsiella, but ovipositor pointed.

\section{Description}

Male (fig. 12). - Forewing length 1.8-2.1 mm, wingspan 3.9-4.7 mm. Head: frontal tuft bicolorous: on crown almost black, on frons yellowish white; collar white. Scape white. Antenna white, relatively long, almost $3 / 4$ forewing length, with 31-41 segments. Thorax and forewing ground colour yellowish white, partly covered with coarse dark brown scales, leaving extensive pale patches, in particular a basal spot, a large costal spot before middle and an elongated dorsal stripe beyond midlength, but occasionally almost completely dark, cilia pale yellow; underside grey-brown with darker margins, no androconial scales. Hindwing grey, no androconial scales. Abdomen almost completely yellowish white to grey, with whitish anal tufts inserted on well sclerotized plates, genitalia narrow.

Female (fig. 13). - Forewing length 1.8-2.1 mm; wingspan 3.8-4.6 mm. Antenna shorter, with 25-31 segments. As male, but forewing usually with more distinct pattern than male, abdomen pale, with remarkable protruding ovipositor, no anal tufts.

Male genitalia (figs. 61-63, 99). - Measurements: see table 3. Vinculum anteriorly slightly bilobed. Uncus with posterior processes well separated, each bilobed. Gnathos with separated slender posterior processes. Valva without distal process, inner margin with a slight bulge in middle, valval tip at inner side dentate, ending in ca 2 teeth; sublateral processes less than half transtilla length. Aedeagus cylindrical; vesica with ca 3-4 long and strong cornuti anteriorly, slightly curved at tip, of about half aedeagus length, a large number of smaller cornuti and in posterior half longer needle like ones; near phallotrema one big triangular tooth at right side; manica small, only covering distal $1 / 3$ of aedeagus, covered with small spines.

Female genitalia (figs. 77, 132, 152). - Apophyses long and slender, forming a protruding ovipositor. Accessory sac almost globular, in posterior half with 3-4 more or less wrinkled sclerotizations set with numerous spines of varying size; ductus spermathecae with 4-5 convolutions after a long straight part.

\section{Biology}

Hostplants. - Quercus ithaburensis subsp. macrolepis**, in Israel, possibly on Q. ithaburensis subsp. ithaburensis (see below).

Leafmine. - A contorted gallery, initially filled with frass, later leaving clear margins. Egg on leaf upperside.

Life-history. - Bivoltine or with more generations. Larvae found in September; adults found in May, June, August and reared in September-October.

\section{Distribution (fig. 169)}

Greece: North, Peloponnesos (Z. \& A. Laštuivka 1998), Rodos, Turkey (new record), possibly Israel: Haifa (as Waldheim) (Amsel \& Hering 1931), who recorded empty mines on $Q$. ithaburensis subsp. ithaburensis (as Q. aegilops).

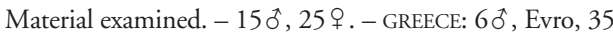
km N Alexandropolis, Kirki, 20-21.viii.1985, A. Moberg (NRMS); 40ิ, 69, Rhodos Kremasti, 3.ix.1973, Quercus macrolepis, e.l. 15.ix.1973-26.ix.1973, J. Klimesch (RMNH, RJ); 3 ô, 13 ㅇ, Rhodos, 5 km S. Rhodos C., 26-30.viii.1991, R. Johansson (RMNH, RJ). - TURKEY: 2 ㅇ, Izmir, $30 \mathrm{~km} \mathrm{NW}$ Bergama, 500-750m, 10-12.v.1993, O. Karsholt (ZMUC); 20ิ, 49, Içel, $10 \mathrm{~km} \mathrm{~S}$ Gülnar, 750m, 30.vi.1993, O. Karsholt (ZMUC, RMNH).

Additional records (A. \& Z . Laštüvka, in litt.). - GREECE: 9ð, 4 오, Messinia, Pirgos, 13.vi.1998; 40, 5 ㅇ, Lakonia, Skoutari, 16.vi.1998.

\section{Stigmella svenssoni (Johansson)}

(figs. 14, 35, 100, 101, 133, 153, 170)

Nepticula svenssoni Johansson, 1971: 249. Holotype ? SWEDEN: Skåne, Hallaröd, 24.vi.1961, I. Svensson, genitalia slide IS 4762 (MZLU) [examined].

Nepticula svenssoni; Borkowski 1972: 774.

Stigmella svenssoni; Emmet 1976a: 243, Johansson \& Nielsen 1990: 231, Puplesis 1994: 159, A. \& Z. Laštüvka 1997: 106.

\section{Diagnosis}

Male with pale grey hindwing without any androconial scales, which separates it from similar $S$. roborella. It is also usually larger and more coarsely scaled than S. roborella and szoecsiella. A previously undescribed diagnostic character is found on the underside of the forewing of the male (fig. 35): in the basal $1 / 2$ of the wing the scale cover is thin, the scales are arranged in some sort of fish-bone pattern, and in the middle of this runs an oblique row of small scales. In the similar species (such as S. roborella: fig. 36 the scaling is thick, and all scales have their tips in the direction of the wingtip.

Male genitalia (measurements: see table 3) characterised by relatively short aedeagus, with long curved cornuti anteriorly. Female genitalia with sclerotized plate in accessory sac and some very small spines pos- 
teriorly; ductus spermathecae long, with about 10 convolutions.

\section{Biology}

Hostplant. - Quercus robur*

Leafmine. - A long, relatively wide gallery with variable width of frass in second half, often dispersed, sometimes linear. Egg on leaf underside. Probably not or hardly possible to separate from S. samiatella and some ruficapitella, but in recent years British authors claim that mines can reliably be separated. However, no clear description of the diagnostic characters has been given. Larva yellow, described in detail by Gustafsson \& Van Nieukerken (1990) who noted a diagnostic character: 2 setal pairs on abdominal segment 9, in contrast to the other species with yellow larvae of which the larva is known, which have 3 pairs.

Life-history. - In northern Europe and Britain believed to be univoltine with occasional partial second generation (Emmet 1976b, 1988, Johansson \& Nielsen 1990), more to the south probably bivoltine. Larvae collected in July-October, occasionally November, adults from May to August, one exceptional finding of a female in an unusually warm January in Germany (see material).

\section{Distribution (fig. 170)}

Widespread in northern half of Europe, but localised: Norway, Sweden (type locality), Finland, Denmark (Buhl et al. 1981), Latvia (Puplesis 1994), Netherlands (Van Nieukerken 1982), Germany (Segerer 1997, Steuer 1998), Slovakia (Tokár et al. 2002), Hungary, France (new record); two isolated records in southern Europe: northern Italy and northern Greece (new record). From Ireland only leafmines recorded (Emmet 1981), up to now no adults found (K. Bond in litt.). Apparently commoner in the northern and western parts of its distribution area.

\section{Remarks}

The previous record from France (Leraut 1997, Van Nieukerken 1996) was a mistake, no material was known to us. The specimen recorded here was found under S. ruficapitella in the Le Marchand collection (MNHN) in January 2003.

The closest relative of $S$. svensson $i$ is most likely the eastern Palearctic S. aladina Puplesis (Van Nieukerken \& Liu 2000): the female genitalia are almost inseparable, and the special scales on the forewing underside also show some resemblance.

Material examined. - 60, $9 ․$ - DENMARK: $1 \hat{0}$, Bornholm, Sorthat, 4.vii.1980, O. Karsholt (ZMUC). - FINLAND: 1 ô, Turku: Puissalo, 27.vi.1970, J. Kyrki (ZMUC). - FRANCE: 10ิ, Calvados, Le Vernay, Quercus robur, e.l. 9.v.1907, Le Marchand (MNHN).- GREAT BRITAIN: $3+$, Cumbria, Armside
Knott, Quercus, e.l. 1-9.v.1980, A. M. Emmet (RMNH). GREECE: 1 으, Evro: $35 \mathrm{~km} \mathrm{~N}$ Alexandropolis, Kirki, 2021.viii.1985, A. Moberg (NRMS). - LATVIA: 10 ${ }^{0}$, 17.vi.1982, Savenkov (ZIAN). - NETHERLANDS: 1 , Gelderland: Hulshorst, 27.iv.1944, L. Vári (ZMAN); $1 \hat{\sigma}$, Noord-Holland: [Callantsoog], Zwanenwater, 28.vi.1984, at light; 1 \% , ibid., 10.vi.1985, at light; 1 , ibid., 1.viii.1989, Quercus robur, e.l. 05.iv.1990; 1 \%, ibid., 14.vi.1986, all leg. J.C. Koster (JCK). SWEDEN: 10ิ, Sk, Sandhammaren, 8.vi.1974, I. Svensson (RMNH); 1 o holotype.

Additional records. - GERMANY: $1+$, Bayern, Regensburg, Asenberg, 9.i.1991 on trunk of Quercus robur, H. Kolbeck (ZSMC, A. Segerer, in litt.).

\section{Stigmella szoecsiella (Borkowski)}

(figs. 15, 64, 65, 78, 102, 134, 154, 171)

Nepticula szoecsiella Borkowski, 1972: 776. Holotype đ: HUNGARY: Budapest, Quercus cerris, e.l. 28.vii.1955, J. Szőcs, genitalia slide Bork. 936 (TMAB) [examined].

Stigmella szoecsiella; Van Nieukerken 1986b: 13, A. \& Z. Laštüvka 1997: 108, Z. \& A. Laštu̇vka 1998: 318.

\section{Diagnosis}

Male similar to $S$. svenssoni and $S$. roborella, but smaller, hindwings darker than in svenssoni and paler than in roborella; special scaling of forewing underside or hindwings absent. Female very similar to other pale headed species, small.

Male genitalia characterised by widened anterior part and curved vesica; separated from similar species S. dorsiguttella, trojana, bicuspidata by presence of a distinct manica and the relatively shorter aedeagus. Female genitalia similar to those of $S$. macrolepidella, but ovipositor less pointed.

\section{Description}

Male (fig. 15). - Forewing length 2.1-2.3 mm, wingspan 4.7-5.0 mm. Head: frontal tuft yellow to orange, ferrugineous; collar yellowish white. Scape yellowish white. Antenna grey brown, $2 / 3$ of forewing length, with 33 segments. Thorax and forewing dark bronzy brown, with purplish sheen; terminal cilia concolorous, slightly paler at tip; underside dark brown, scales normal. Hindwing pale grey. Abdomen not studied, with anal tufts (colour unknown) inserted on well sclerotized plates.

Female. - Forewing length $2.2-2.3 \mathrm{~mm}$; wingspan 4.8-5.2 mm. Antenna with ca 25 segments. As male, abdomen not studied.

Male genitalia (figs. 64, 65, 102). - Measurements: see table 3 . Vinculum anteriorly slightly bilobed. Uncus with widely separated, rather short horns. Gnathos with long, widely separated posterior processes. Valva with pointed distal process less than valva length; inner margin almost straight, ending in rounded lobe; sublateral processes about $1 / 4$ transtilla length. Aedeagus short, basally widened, flask-shaped; vesica with 

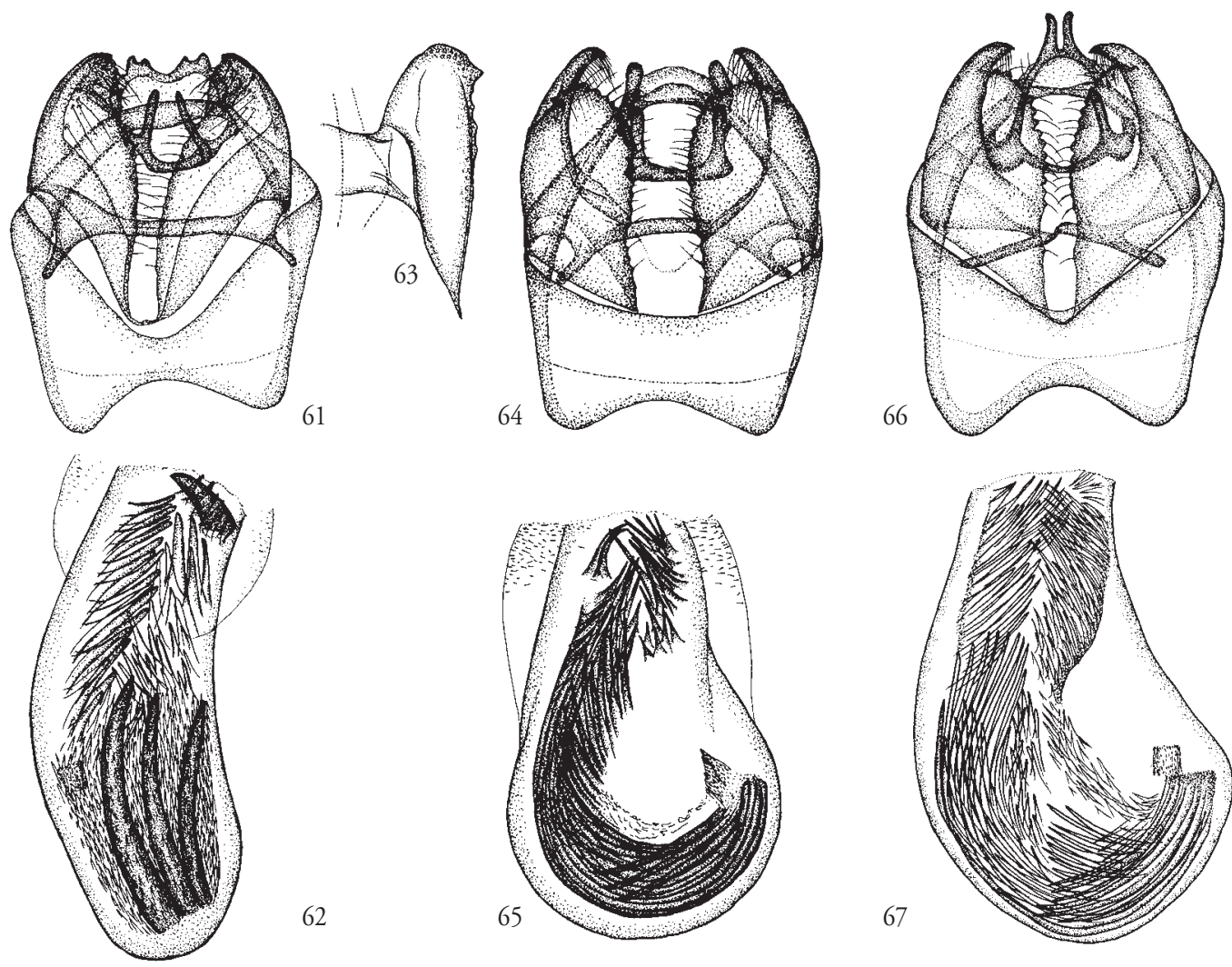

62
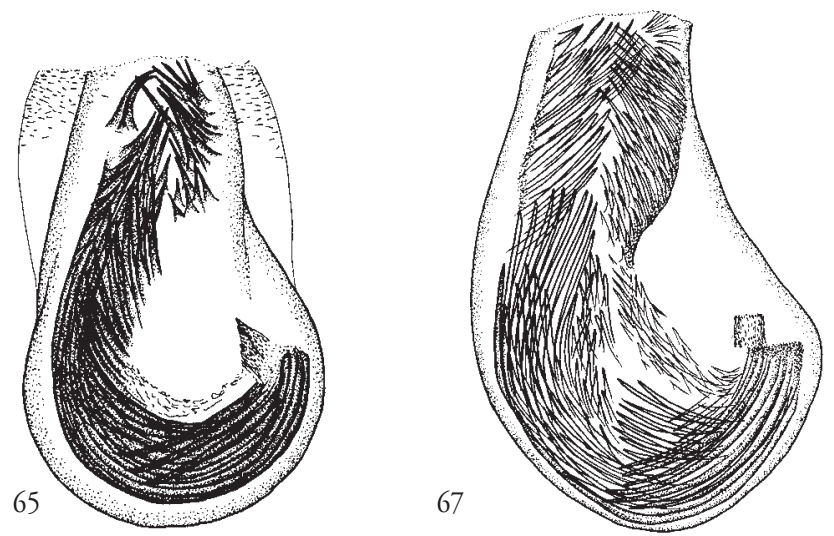

single coil anteriorly, with group of very long curved cornuti in basal half and many small ones; distally a small group of ca 15-20 needle like cornuti at right side and a few at left, near phallotrema; manica present, covering distal half of aedeagus, covered with small spines and pectinations.

Female genitalia (figs. 78, 134, 154). - Accessory sac with many longer spines posteriorly; none anteriorly; ductus spermathecae with 3-3.5 convolutions, ca twice as long as bursa.

\section{Biology}

Hostplants. - Quercus cerris**, Q. trojana (A. \& Z. Laštúvka 1997).

Leafmine. - A gallery with thin frass first and dispersed frass later, very similar to that of $S$. zangherii. Egg on leaf upperside. Larva yellow.

Life-history. - Larvae found in July and September, adults in June-August.
Distribution (fig. 171)

A rare species with very few records: Hungary, Italy (new record), Greece (Z. \& A. Laštuivka 1998), Turkey (new record). The record from the Ukraine (Crimea) by Puplesis (1994) is based on misidentified $S$. dorsiguttella, q. v. Highest altitude $1700 \mathrm{~m}$ in Turkey.

Material examined. $-3 \hat{0}, 2+$. - GREECE: $1+$, Ioannina, 4 km SW Geroplatanos, 800m, 12.vii.1998, D. Nilsson \& B. Skule (ZMUC). - HUNGARY: 10 , Holotype; 1 ㅇ paratype, Szár, Quercus cerris, e.l. 5.iv.1965, J. Szőcs. - ITALY: 10ે, Chieti, Vasto, Fiume Sinello, 19.vii.1998, P. Triberti (PT). TURKEY, $1 \hat{\sigma}$, Nigde: $40 \mathrm{~km}$ NW Nigde, Sivrihisar pass, 1700m, 4.viii.1989, Fibiger \& Esser (ZMUC).

\section{Stigmella zangherii (Klimesch)}

(figs. 16, 17, 66, 67, 79, 103, 104, 135, 155, 172)

Nepticula zangherii Klimesch, 1951a: 61. Syntypes 40 : ITALY: Farrazano near Meldola (Forli), ca 60 m, 28.viii. 1949, at light, P. Zangheri (ZSMG) [not examined]. 

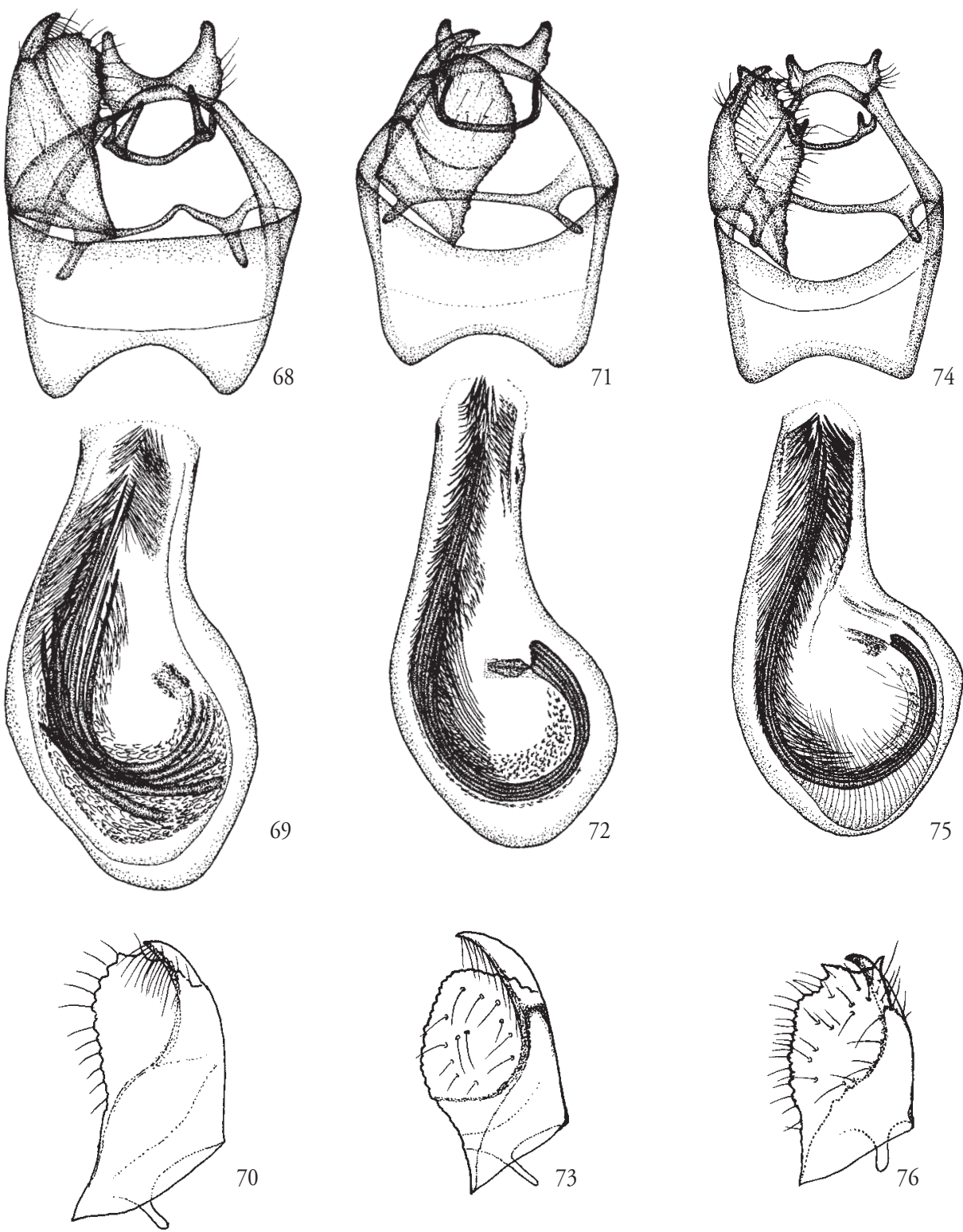

Figs. 61-76. Stigmella spp., male genitalia, capsule $(61,64,66,68,71,74)$, aedeagus $(62,65,67,69,72,75)$ and valva, lateral (63) or dorsal aspect $(70,73,76)$ - - 61-63, S. macrolepidella, Greece, Rhodos, slide RJ 1786+2268 (63); 64, 65, S. szoecsiella, Italy, slide EvN3345; 66, 67, S. zangherii, Turkey, slides RJ 1751, RJ2260 (aedeagus); 68-70, S. dorsiguttella, Greece, slide RJ 1750; 71-73, S. trojana, Greece, paratype, slide EvN3374; 74-76, S. bicuspidata, holotype, slide EvN 3278. 
Nepticula zangherii; Szőcs 1968: 229 (description female and biology), Johansson 1971: 249, Borkowski 1972: 775.

Stigmella zangherii; Zangheri 1969: 1014, Van Nieukerken 1986b: 13, A. \& Z. Laštúvka 1990: 187, 1997: 107.

[Nepticula subnitidella sensu Zeller 1848: 305. Misidentification.]

[Trifurcula minimella Rebel, 1926: (110) partim. Misidentification.]

\section{Diagnosis}

Males recognised by head black on crown and distinct yellow to cream androconial scales on hindwing and forewing underside. S. dorsiguttella has similar but darker androconials and a pale head. Females resemble $S$. basiguttella, $S$. trojana and to a lesser extent $S$. macrolepidella.

Male genitalia easily recognised by closely placed uncus lobes, otherwise rather similar to $S$. dorsiguttella, trojana and bicuspidata. Female genitalia characterized by the accessory sac, almost completely covered with small spines and one group of larger spines.

\section{Description}

Male (fig. 16). - Forewing length 2.0-2.4 mm, wingspan $4.2-5.5 \mathrm{~mm}$. Head: frontal tuft brown to black, on frons white to ochreous; collar yellowish white. Scape white. Antenna brown, of $2 / 3$ forewing length, with 31-38 segments. Thorax and forewing dark brown, usually with a pale patch at tornus, but sometimes pale pattern becoming predominant; underside with elongate patch of deep yellow to ochreous white androconial scales, only wingtip without. Hindwing covered on both sides with deep yellow to ochreous white androconial scales. Abdomen dorsally brown, ventrally almost yellowish white, with yellowish white anal tufts inserted on well sclerotized plates.

Female (fig. 17). - Forewing length 1.8-2.3 mm; wingspan 3.9-4.9 mm. Antenna with 25-28 segments. As male, but androconial scales and anal tufts absent. Ovipositor slightly pointed.

Male genitalia (figs. 66, 67, 103, 104). - Measurements: see table 3. Vinculum anteriorly bilobed. Uncus with posterior horns close together, almost parallel or forming a ' $\mathrm{V}$ '. Gnathos with separated posterior processes, usually converging towards tip. Valva with short, curved distal process, about $1 / 6$ of valva length; inner margin evenly curved, not forming a distinct lobe; sublateral processes less than half transtilla length. Aedeagus anteriorly very broad; vesica with single coil anteriorly, some very long and strong curved cornuti following the coil, a large number of smaller cornuti alongside these long ones, following the curvature of the vesica; distally in groups arranged left and right; manica absent.

Female genitalia (figs. 79, 135, 155). - Accessory sac with many small spines with short point and fewer long spines posteriorly; ductus spermathecae with about 6 convolutions.

\section{Biology}

Hostplants. - Quercus cerris**, Q. ithaburensis subsp. macrolepis (A. \& Z. Laštüvka 1997), Q. pubescens (Borkowski 1972), Q. suber, Q. trojana, (A. \& Z. Laštüvka 1997).

Leafmine. - A gallery with narrow frass first, later often dispersed. Egg on leaf upperside. Mine not to be distinguished from S. szoecsiella and various other species. Larva yellow.

Life-history. - Bivoltine, larvae recorded in June, late August and October, adults from May to September.

\section{Distribution (fig. 172)}

Widespread in south-eastern Europe and Turkey, in the north up to the Czech Republic and Slovakia (Laštuivka \& Laštuivka 1990), in the west a single specimen from south eastern France (Borkowski 1972), further known from eastern Austria (A. \& Z. Laštu̇vka 1997), Hungary, Italy, Sicily, Slovenia, Croatia, Yugoslavia, Greece (A. \& Z. Laštủvka 1997) and Turkey (new record). From sea level up to $1800 \mathrm{~m}$ in Turkey.

Material examined. - 220ิ, 20․ - AUSTRIA: 19 , Niederösterreich, Hof am Leithagebirge, $4 \mathrm{~km} \mathrm{~S}$ Mannersdorf, 16.x.1992, Quercus cerris, e.l. 23.iii.1993, E. J. van Nieukerken (RMNH). - CROATIA: 1 $\sigma^{\star}$, Krk, Kampelje, 17. viii.2001, G. Baldizzone (GB); 10, Krk, Misucaynica, 30. vi.1976, E. Jäckh; 10ิ, Krk, Vrbnik, 29.vi.1976, E. Jäckh (USNM). - CZECH REPUBLIC: 1 \% , Moravia, Lednice, $2.5 \mathrm{~km}$ S, 'Liechtenstein' forest, 3.x.1992, leafmines, Quercus cerris, e.1. 23.iii.1993, E. J. van Nieukerken (RMNH). - GREECE: 10 , Evro, 35 km N Alexandropolis, Kirki, 20-21.viii.1985, A.

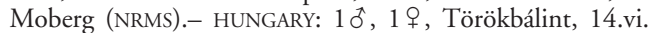
1974, Quercus cerris, e.l. 29.vi.1974, J. Szőcs (RJ). - ITALY: 1 ․ L’Aquila, Parce Naz. d'Abruzzo, Valle F. Sangro, Casoni, versante Nord, $1100 \mathrm{~m}, 13$. vii.1998, P. Triberti (PT); 10ิ, Latina, Monti Aurunci, $5 \mathrm{~km} \mathrm{~N} \mathrm{Itri,} \mathrm{850m,} \mathrm{4-11.}$ viii. 1972, R. Johansson; 1 क , Latina, Monti Aurunci, 6 km N Itri, 600m, 15.viii.1972, R. Johansson (RJ); $1 \hat{\delta}$, Potenza, Mte Vulture, dint. Laghi di Monticchio, 850m, 17.v.1967, F. Hartig; 10 , ibidem, 750m, 16.v.1966, F. Hartig; 10 , ibidem, 4.v.1968, F. Hartig (MRSN). - SLOVAKIA: 19 , Volkovce, 4 km NW Kozárovce, 7.x.1992, leafmines, Quercus cerris, e.l. 23.iii.1993, E. J. van Nieukerken (RMNH). TURKEY: $8 \delta, 13+$, Adana, Taurus, $4 \mathrm{~km}$ NE Feke, $1000 \mathrm{~m}$, 7.ix.1983, G. Derra (GD, RMNH); 2 $\widehat{0}, 1$, Isparta, Aksehir 30 km SW, Sultan Daglari, 1500m, 30-31.viii.1997, K. Nupponen \& J. Junnilainen (JJ, RMNH); $2 \hat{\sigma}$, Içel [Mersin], Güzeloluk NW Erdemli, 1400m, 16.vii.1986, M. Fibiger; 1 ㅇ, Nigde, 40 km NW Nigde, Sivrihisar pass, 1700m, 4.viii. 1989, Fibiger \& Esser (ZMUC).

Additional records (A. \& Z . Laštúvka, in litt.). - CROATIA: Istra, Rabac; Dalmacia, Zadar and Oračac. - GREECE: $2 \hat{0}$,

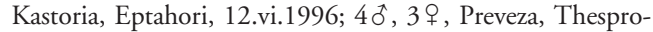
tiko, 11.vi.1997; 6 0 , 5 9 , Etolia, Skourtou, 12.vi.1997; 40ิ, 4ㅇ, Lakonia, Nea Marathea, 19.vi.1996. - ITALY: Campania, San Salvatore Telesino; Firenze, San Benedetto in Alpe; Albáno di Lucania. - ITALY, SICILY: Castelbuono; Francavilla and Ficussa. - Slovenia: Maribor. - YUgoslavia: Crna Gora, Petrovac. 
11. Stigmella dorsiguttella (Johansson)

(figs. 18, 19, 68-70, 106, 108, 136, 137, 156, 173)

Nepticula dorsiguttella Johansson, 1971: 251. Holotype + , SWEDEN: Småland, Högsby, 22.vi.1970, R. Johansson, Genitalia slide RJ1035 (RJ) [examined].

Nepticula dorsiguttella; Borkowski 1972: 778.

Stigmella dorsiguttella; Pröse 1984: 107, Johansson \& Nielsen 1990: 232, Puplesis 1994: 161, A. \& Z. Laštüvka 1997: 109 .

[Stigmella szoecsiella; Puplesis 1994: 160. Misidentification.]

\section{Diagnosis}

Males of $S$. dorsiguttella are usually immediately recognized by the combination of a pale head and the very conspicuous yellow to orange scaling of hindwing. In some specimens this scaling may, however, be more brownish (perhaps as a result of ageing), then it more closely resembles $S$. eberhardi, which has still darker brown bronze scales on hindwing. On the underside of the forewing dorsiguttella has a distinct patch of androconials reaching about $2 / 3$, whereas eberhardi has a uniform cover of dark brown scales. The forewing of dorsiguttella usually has a pale stripe along dorsum (hence its name). Males of $S$. zangherii could be confused because of similar, but usually paler androconials, but the head in zangherii is usually black. Females resemble most other pale-headed species, but dorsiguttella is the only one with a pale dorsum.

Male genitalia (measurements see table 3 ) very similar to those of $S$. trojana and bicuspidata, the short valval process and wide aedeagus are characteristic. Female genitalia characterised by the almost globular bursa with longer spines mostly at one side, and a group distally in ductus; ductus spermathecae with about 5 convolutions.

\section{Biology}

Hostplants. - Quercus petraea**, Q. robur**.

Leafmine. - A relatively short gallery, usually with thin frass line, dispersed in last part. Egg position unknown. Larva yellow, described in detail by Gustafsson \& Van Nieukerken (1990).

Life-history. - The few known larvae were found in July, August and September, adults collected from May to September, probably bivoltine in the south and univoltine in the north.

\section{Distribution (fig. 173)}

Widespread but very local in central, eastern and southern Europe and southwestern Asia: southeastern Sweden, Germany (Pröse 1984, here for the first time recorded from Rheinland-Pfalz), Poland (Buszko 1987), Czech Republic (A. \& Z. Laštúvka 1990), Slovakia (A. \& Z. Laštuivka 1991), Austria (Kasy 1983), France, Portugal (new record), Spain (new record), Italy (new record), Slovenia and Croatia (A. \& Z. Laštủvka 1997), Ukraine (Puplesis 1994), Greece (new record) and Turkey (new record). In the north occurring in warm places only. Highest altitude: $2400 \mathrm{~m}$ in Turkey.

\section{Remarks}

Puplesis (1994: 160) and Budashkin (1988) identified Crimean specimens with dark hindwings as $S$. szoecsiella. However, the male genitalia depicted by Puplesis are indistinguishable from $S$. dorsiguttella, and $S$. szoecsiella also has no androconials.

Material examined. - 29ð, 16․ - AUSTRIA: 20, Niederösterreich: Glaslauterriegel, Gumpoldskirchen, 1.viii. 1975, 22.viii.1981, F. Kasy (NMW); 10 , 1 을 Niederösterreich: Hundsheimer Berg S., 17.vi+8.vii.1980, F. Kasy (NMW). - CROATIA: 10 , Sucurac, Dalmatia, ix.1923, Novak (NMW) [misidentified paralectotype of Trifurcula minimella Rebel]. - CZECH REPUBLIC: $1+$, Moravia, Detkovice, 28.viii. 1989, Quercus petraea, e.l. iii.1990, A. Laštüvka; 1 ô, Moravia, Milovice, 10.ix.1980, Quercus robur, e.l. iii.1981, A. Laštuivka (RMNH). - FRANCE: 1 ô, Drôme, Les Prés, 2 km N. Valdrôme, 800m, 21.viii.2002, at light, E. J. van Nieukerken (RMNH). - GERMANY: $1 \delta$, Bayern, Münnerstadt, Dianonruh, Unterfranken, 26.vi.1986, H. Hacker (GD); 60, 1 오, Bayern, Stettfeld, Unterfranken, Kohlberg, 9.vi.1977, 2.vi.1978, G. Derra (GD, RMNH); 10 , Rheinland-Pfalz, Bad Münster/Stein, NSG Rotenfells, 4.v.2003, W. Biesenbaum (WB); 1 , Thüringen, Bad Blankenburg, Buntsandstein, 16.viii. 1968, H. Steuer; 1 , , Thüringen, Bad Blankenburg, Muschelkalk, 19.vii.1989, H. Steuer (HS).- GREECE: 10 t 3 क , Evro: $35 \mathrm{~km}$ N Alexandropolis, Kirki, 500m, 20-21. viii.1985, M. Fibiger, A. Moberg (ZMUC, NRMS); 1 \% , ibid., 8.vii.1986, M. Fibiger (RJ); 10 , Thessaloniki: $12 \mathrm{~km} \mathrm{NW}$ Gumenissa, $1100 \mathrm{~m}$, 26.vii.1986, M. Fibiger (RJ). - ITALY: 1 ㅇ, Torino: Pralormo, Mad. d. Spina, 321m, 24.vi.1991, G. Delmastro (GB). - PORTUGAL: 1 + , Alentejo: Galegos, Portalegre, 16.ix.1995, M. F. V. Corley (MC). - SLOVAKIA: 30े, 1 , , Viniansky hrad, 31.vii.1993, 17.vii.2000, Z. Tokár; 1ㅇ, NR Kasvár, 10.vii.1993, Z. Tokár (RMNH). - SPAIN: 1 ô, Teruel, near Puerto de Orihuela, 1600m, 28.viii.1984, P. Skou \& M. Kavin (ZMUC). - SWEDEN: $3 \hat{0}$ paratypes, 1 q holotype, Småland, Högsby, 22.vi.1970, R. Johansson; 1 ઈ, same data but 21.vi.1975; 1 đิ, Småland, Skoghult, 17.vi. 1972, R. Johansson; 1 ô, Öland, Högsrum, 15.vi.1974, R. Johansson (RJ). TURKEY: 1 đิ, 1 \% , Agri, Tahir Gecidi, 2400 m, 18.ix.1985, G. Derra (GD). - UKRAINE: 7ठ․ Crimea, Karadag, $20 \mathrm{~km} \mathrm{W.} \mathrm{of}$ Feodosia, 13.viii.1985, 12-21.vii. 1987, Budashkin, R. Puplesis (ZIAN) [partly misidentified as S. szoecsiella].

Additional records (A. \& Z . Laštu̇vka, in litt.). - GERMANY: Rheinland-Pfalz, Eisenberg. - GREECE: Kalavrita. SPAIN: 10, Granada, El Molinillo, 1200m, 28.vi.1992.

\section{Stigmella trojana Z. \& A. Laštüvka} (figs. 20, 21, 71-73, 80, 109, 110, 138, 157, 174)

Stigmella trojana Z. \& A. Laštüvka, 1998: 313. Holotype ơ: GREECE: Kozani, Galani, ex larva 1997, Raupe 9.vi.1997, Quercus trojana (AL) [paratypes examined]. 


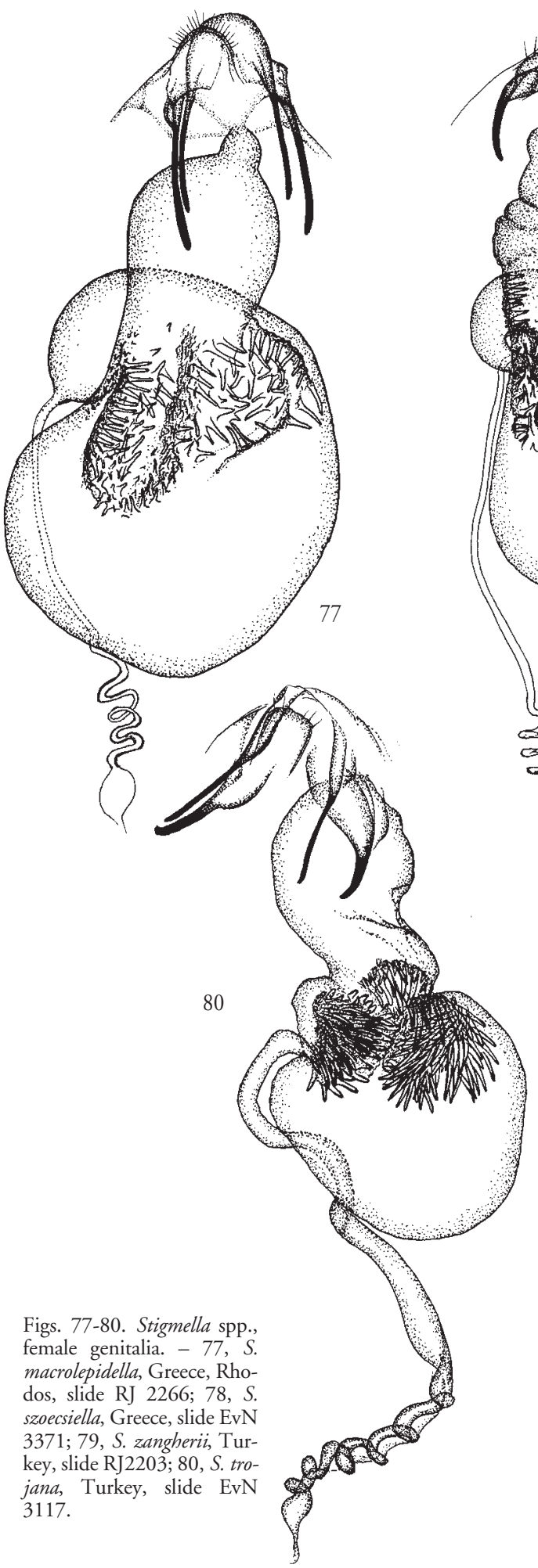

Figs. 77-80. Stigmella spp., female genitalia. - 77, $S$. macrolepidella, Greece, Rhodos, slide RJ 2266; 78, S. szoecsiella, Greece, slide EvN $3371 ; 79$, S. zangherii, Turkey, slide RJ2203; 80, S. trojana, Turkey, slide EvN 3117.
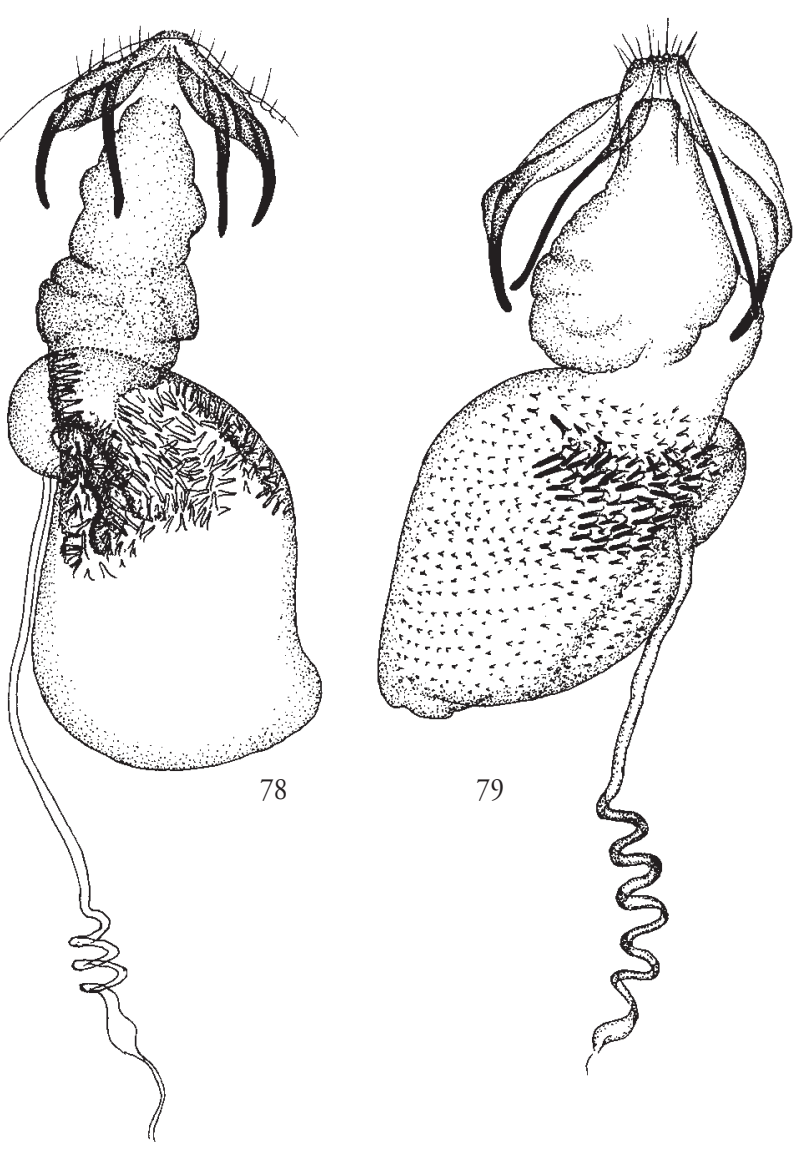

\section{Diagnosis}

Males somewhat resemble $S$. basiguttella and $S$. samiatella, but have usually a paler dorsum and a more mottled appearance; also the spatulate scales along hindwing costa (to be seen from underside!) distinguish trojana. Females can be confused with other black-headed species, particularly S. atricapitella and S. zangherii.

Male genitalia separated from similar S. bicuspidata by smoothly rounded inner lobe of valva, from very similar $S$. dorsiguttella by shorter inner lobe, not reaching tip of distal process. Female genitalia characterised by the arrangement of the spines in the globular accessory sac on two bulbous lobes.

\section{Description}

Male (fig. 20). - Forewing length 2.1-2.3 mm, wingspan 4.4-5.0 mm. Head: frontal tuft fuscous to black, occasionally a few yellow scales; collar white. Scape white. Antenna grey brown, $2 / 3$ of forewing length, with 34-36 segments. Thorax and forewing dark grey brown, hardly shining at tip, along dorsum 
usually paler, almost white; terminal cilia concolorous, slightly paler at tip; underside dark grey brown, with basal elongate yellowish grey, pale spot of indistinct androconial scales. Hindwing pale grey, along dorsum in basal third with long spatulate androconial scales, inserting in patch on underside forewing; usually only visible from underside. Abdomen dark grey, with pale yellowish anal tufts inserted on well sclerotized plates.

Female (fig. 21). - Forewing length 2.0-2.3 mm; wingspan 4.5-4.9 mm. Antenna with 21-26 segments. As male, but patch underside forewing and androconial scales absent; forewing sometimes completely unicolorous; abdomen dark grey, without anal tufts.

Male genitalia (figs. 71-73, 109, 110). - Measurements: see table 3 . Vinculum anteriorly slightly bilobed. Uncus with widely separated conical and curved horns. Gnathos with long slender, widely separated posterior processes. Valva with pointed distal process, slightly less than valva length; inner margin forming a distinct inner lobe, which does not reach to tip of distal process; sublateral processes short, less than $1 / 4$ transtilla length. Aedeagus long and anteriorly widened; vesica with single coil anteriorly, several very long and strongly curved cornuti following the coil, with several associated small cornuti, more posteriorly with rows of shorter spines at both sides, distally also a row at right hand side of aedeagus; manica absent.

Female genitalia (figs. 80, 138, 157). - Accessory sac with many longer spines posteriorly, all directed anteriorly, inserted on two cushion-shaped bulbous lobes; ductus spermathecae with 5 convolutions, at least $2 \times$ as long as bursa.

\section{Biology}

Hostplant. - Quercus trojana**; since the record in Turkey is outside the range of $Q$. trojana (see fig. 174), it probably also feeds on another oak.

Leafmine. - A gallery, according to the original description not different from S. zangherii or S. szoecsiella, which feed on the same host, egg position not noted. Larva yellow.

Life-history. - Probably bivoltine, larvae found in June, adults emerging almost immediately in June; also found in July and October.

\section{Distribution (fig. 174)}

Northern Greece and Turkey (new record). From sea level up to $2400 \mathrm{~m}$ in Turkey.

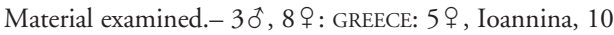
km NE Geroplatanos, by Konitsa, 24.vii.1990, M. Fibiger (ZMUC, RMNH); 1 ô, Grevená, Miliá, 8.vi.1998, A. \& Z. Laštủvka (AL); 1 ô, 2 ㅇ (paratypes), Kozani, Galáni, 10.vi.1997, Quercus trojana, e.l. vi.1997, A. \& Z. Laštüvka (RMNH, AL); 1 ô (paratype), Préveza, Thesprotiko, 14.vi.1996, A. \& Z. Laštùvka (AL). - TURKEY: 19: Agri, Tahir Gecidi, 2400 m, 10.x.1985, G. Derra, Gen. slide EvN 3117 (GD).

\section{Stigmella bicuspidata sp. n.}

(figs. 22, 74-76, 111, 112, 174)

Type material. - Holotype $\widehat{0}$ : TURKEY: Prov. Nigde: $40 \mathrm{~km} \mathrm{NW}$ Nigde, Sivrihisar pass, 1700m, 4.viii.1989, Fibiger \& Esser, genitalia slide EvN 3278 (ZMUC). - Paratypes, 20: TURKEY 10 , Gümüshane: Pirnakapan, 1800m, 19.vii.1989, Fibiger \& Esser, genitalia slide EvN 2848 (ZMUC); 10 , Adana: Taurus, 4 km NE Feke, 7.ix.1983, G. Derra, genitalia slide EvN 2781 (GD).

\section{Diagnosis}

Externally most similar to $S$. trojana, which usually has a paler hindwing and a distinct paler area on forewing underside. Males separated from other similar species, such as basiguttella and samiatella by the narrow spatulate scales along hindwing costa.

Female unknown.

Male genitalia resembling $S$. dorsiguttella, S. szoecsiella and $S$. trojana. It differs from szoecsiella by the absent manica, from trojana by the shape of valva and uncus and from dorsiguttella by narrower uncus lobes and shape of valvae.

\section{Description}

Male (fig. 22). - Forewing length 2.1-2.2 mm, wingspan 4.9-5.0 mm. Frontal tuft yellow to black on vertex and paler lower down, scape and collar white, antenna with 32 segments. Thorax and forewings bronze brown. Hindwing grey, along costa with narrow spatulate androconials, usually only visible from underside; underside forewing grey brown. Anal tufts not studied, inserted on well sclerotized plates.

Female. - Unknown.

Male genitalia (figs. 74-76, 111, 112). - Measurements: see table 3 . Vinculum anteriorly hardly excavated. Uncus with widely separate, curved processes. Gnathos with relatively short, widely separated posterior processes. Valva with rather long pointed distal process, hidden behind posterior lobe of inner margin; inner margin convex, distally with pronounced bicuspidate lobe; sublateral processes about 1/4 transtilla length. Aedeagus long and anteriorly widened; vesica with single coil anteriorly, with wrinkled surface, several very long and strongly curved cornuti following the coil, with several associated small cornuti, more posteriorly with rows of shorter spines at both sides; manica absent.

\section{Biology}

Host and immature stages unknown. Adults found from July to September.

\section{Distribution (fig. 174)}

Only known from Turkey, from three widely separate localities. 


\section{Etymology}

An adjective, bicuspidatus: with two teeth, because of the valva with two teeth-shaped points. Here in female form because of gender of Stigmella.

\section{Stigmella ruficapitella (Haworth)}

(figs. 23, 24, 105, 106, 139, 158, 175)

Tinea ruficapitella Haworth, 1828: 586. Lectotype 9 : ENGLAND: [London], Stainton Coll., B.M. $q$ genitalia slide No. 14862. (designated by Johansson 1971: 256) [examined].

Nepticula ruficapitella; Petersen 1930: 45, fig. 5 (male genitalia), Johansson 1971: 255, Borkowski 1972: 784.

Stigmella ruficapitella; Emmet 1976a: 239, Johansson \& Nielsen 1990: 233, Puplesis 1994: 155, A. \& Z. Laštúvka 1997: 110 .

[Nepticula samiatella; Herrich-Schäffer 1855: 348 (partim, male), Petersen 1930: 46, fig. 8 (male genitalia), Beirne 1945: 198, fig. 13 (male genitalia), misidentification.]

[Stigmella atricapitella; Beirne 1945: 198, fig. 13 (male genitalia), misidentification.]

\section{Diagnosis}

Males easily recognised by combination of black head and the distinct patch of relatively short androconial scales on hindwing: only in basal half and reaching $1 / 3$ into the fringe, the other species with similar androconials (S. atricapitella, suberivora, ilicifoliella, cocciferae) have these scales much longer and extending over most of hindwing. Female resembling other red-headed species with uniform forewings: $S$. suberivora, ilicifoliella, cocciferae, kasyi, szoecsiella, svenssoni, roborella, eberhardi; it can be distinguished from the most common roborella by the blunt abdomen versus the pointed one in S. roborella; most other red-headed species have more antennal segments (23-27 in S. ruficapitella), except S. szoecsiella and dorsiguttella, there is some overlap with $S$. svenssoni.

Male genitalia (measurements see table 3): manica conspicuous, almost twice as wide as aedeagus. Female genitalia: accessory sac an elongated pear-shaped sac, with wrinkled walls, many short spines confined to patch in posterior part, in top inserted on a distinct sclerotization; ductus spermathecae with 5 convolutions.

\section{Biology}

Hostplants. - Quercus cerris (Szőcs 1977), Q. petraea**, Q. pubescens (Borkowski 1972), Q. robur**. Most abundant on $Q$. robur and petraea. Records on Castanea (Buhr 1940) require confirmation, such mines may well belong to $S$. samiatella.

Leafmine. - A gallery with frass first in narrow midline, later in a broad band. Egg usually on leaf upperside, but also found on underside. A Dutch sample of 158 specimens, shown to belong to one species by their allozymes, had $78 \%$ of eggs on leaf upperside (Cronau
\& Menken 1990: published under the name samiatella, but here believed to be ruficapitella, see below); all over leaf, but less so against veins. Larva yellow, described by Gustafsson \& Van Nieukerken (1990).

Life-history. - Bivoltine, larvae June to November, most abundant in October; adults April-June and July-September, one of the earliest flying oak mining species, often just before the opening of leaves.

\section{Distribution (fig. 175)}

Throughout northern and central Europe, but almost absent in the Mediterranean region with the exception of Mount Olympus in Greece and Trieste. In the North and West one of the commonest species. Recently recorded from Russia (Shmytova 2002). New records from Bosnia and Greece. Highest altitude on Mount Olympus, but without exact altitude known.

\section{Remarks}

The species identified by Cronau \& Menken (1990) as samiatella in their allozyme analysis, is most likely S. ruficapitella (see under samiatella). Descriptions under the name ruficapitella prior to 1971 very often refer to $S$. roborella. However, the male genitalia under that name, illustrated by Beirne (1945), belong to $S$. hemargyrella.

Material examined. - 98ð, $74 \%$. - AUSTRIA: $19 .-$ BELGIUM: 7ð, 8 + . - BOSNIA: S. of Han Knezica, ca $11 \mathrm{~km} \mathrm{~N}$ of Prijedor, 17.x.1983, Quercus robur, e.l. 15.iv.1984, E. J. van Nieukerken \& J.J. Boomsma (RMNH). - FRANCE: 50, 5 ㅇ. GERMANY: $8 \hat{\sigma}, 19+$. . GREAT BRITAIN: $2 \hat{\jmath}, 1+$. - GREECE: Larissa, Olympos, 700-2100m, 21-26.v.1990, O. Karsholt (ZMUC, RMNH). - HUNGARY: 2ㅇ. - LATVIA: 40, 2 ㅇ. LITHUANIA: $4 \hat{\sigma}, 1 \%$. - NETHERLANDS: $56 \hat{0}, 33 \%$. - SLOVAKIA: $3 \hat{\jmath}$. - SWEDEN: many, not counted. - UKRAINE: $4 \hat{0}$, Kiev, 17-25.viii.1934, Lebedev (SIZK).

\section{Stigmella atricapitella (Haworth)}

(figs. 25, 26, 113, 114, 140, 159, 176)

Tinea atricapitella Haworth, 1828: 585. Syntype 9 : ENGLAND: [London], Stainton coll. (BMNH) [examined; abdomen absent, poorly preserved].

Nepticula discrepans Sorhagen, 1922: 44. Holotype leafmine: GERMANY: Hamburg, vii, Quercus, Sorhagen [destroyed] (synonymised by Van Nieukerken \& Johansson 1987).

Nepticula atricapitella; Herrich-Schäffer 1855: 347; Johansson 1971: 253, Borkowski 1972: 782.

Stigmella atricapitella; Emmet 1976a: 239, Johansson \& Nielsen 1990: 234, Puplesis 1994: 156, A. \& Z. Laštüvka 1997: 111.

[Stigmella samiatella; Vári 1950: 180 [partim], 183, fig. 1 (female genitalia). Misidentification.]

\section{Diagnosis}

Male very characteristic by black head and long androconials on hindwing, reaching to $2 / 3$ of fringe along 
most of hindwing. Only rare dark-headed specimens of the evergreen oak miners (only known from S. ilicifoliella) could be confused, but they have usually longer antennae with more segments (30-35 in S. atricapitella, 39-50 in ilicifoliella).

Females very similar to $S$. samiatella and the rarer, uniformly coloured $S$. basiguttella or trojana.

Male genitalia (measurements see table 3): manica inconspicuous, narrow. Female genitalia: an elongated accessory sac with some more or less sclerotized folds is characteristic; spines of different length, the longer ones on a rounded lobe; ductus spermathecae with 5 convolutions.

\section{Biology}

Hostplants. - Quercus cerris (Szöcs 1977), Q. pe-

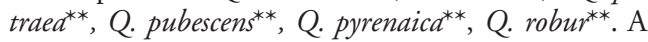
specimen reared from Castanea sativa (Agassiz 1988) proved to be misidentified S. samiatella (J. Langmaid in litt. 2003). In the Czech Republic only found on $Q$. pubescens (A. \& Z. Laštüvka in litt.).

Leafmine. - A contorted gallery, frequently near leaf margin and confined to small area; frass usually in a narrow line, often broader in second part. Egg on either side of leaf. Larva pale yellow or almost white, prothorax with dark coloured sclerites (always?), which may help in distinguishing the mine from the similar S. roborella (see photographs in Huisman et al. 2001). Larva described in detail by Gustafsson $\&$ Van Nieukerken (1990).

Life-history. - Bivoltine with larvae in June-July, September-November, and adults from May to early October.

\section{Distribution (fig. 176)}

Throughout Europe, with a more or less AtlanticMediterranean distribution type: becoming rarer or absent in the North, with only very scattered records in southwestern Scandinavia. The records in Lithuania are outliers in that respect: it is very scarce in that country (R. Puplesis in litt., Diškus 2003), but it may have been overlooked in Poland. In the northern part of its range usually in warm places, isolated oaks or forest margins; in the south one of the commonest species. No positive records from Albania, Belarus, Bosnia, Finland, Latvia, Moldova, Poland or Russia. New (or confirmed) records from Bulgaria, Georgia, Italy: Sicily, Macedonia, Portugal, Turkey and Yugoslavia. Records from Estonia (Jürivete et al. 2000) should be checked, if they are based on Petersen (1930), they are wrong. The latter author illustrated male ruficapitella under this name. Occurs also as the only oak feeding species commonly on Madeira, most likely an introduced insect (Aguiar \& Karsholt in press). Highest altitude: $2400 \mathrm{~m}$ in Turkey.

\section{Remarks}

Because of the poor condition of the single remaining syntype, the identity of $S$. atricapitella was settled by Johansson (1971) in favour of current practice. This follows Herrich-Schäffer (1847-1855), who described Nepticula atricapitella as the British species with long androconial scales.

Material examined. - $1160^{\circ}, 68 \%, 2$ adults. - AUSTRIA: $7 \hat{\sigma}, 8+$. - BELGIUM: $3 \hat{0}, 2$ ․ - BULGARIA: $2 \hat{0}$, Burgas, 40 km SE, Ropotamo, 26-27.vi.2001, J. Junnilainen (JJ, RMNH). - CROATIA: 18 ô, Krk, 1975-2001, E. Jäckh, G. Baldizzone (USNM, GB, RMNH). - FRANCE: 160, $9 \%, 2$ adults. - GERMANY: $2 \hat{\jmath}, 5 q .-$ GEORGIA: $5 \hat{\jmath}$, Lagodekhi, 300m, 2122.vii, 20-30.viii.1986, Kozlov, Seksyaeva, Sinev (ZIN). GREAT BRITAIN: $1 \hat{\delta}$. - GREECE: $1 \hat{\delta}, 8 \%$, Evro, $35 \mathrm{~km} \mathrm{~N}$ Alexandropolis, Kirki, 500m , 20-21.viii.1985, A. Moberg (NHRS); $8 \hat{o}, 12$ ㅇ, Larissa, Olympos, 700-2100m, at light, 21-26.v.1990, O. Karsholt (ZMUC, RMNH). - ITALY: $16 \delta^{\star}$, 7․ - LITHUANIA: $2 \hat{\sigma}, 3$ 9 . - NETHERLANDS: $3 \hat{\sigma}, 69$. - PORTUGAL: $1 \delta$, Beira Alta, Caldas de Manteigas, $850 \mathrm{~m}$, 7.ix. 2001, M. F. V. Corley (MC). - PORTUGAL, MADEIRA: 10 , Santana, 600m, 3.viii.1999, E.E.G. van Riel (CVDB). - SLOVAKIA: $2 \hat{\sigma}, 2{ }^{\circ} .-$ SPAIN: $2 \hat{0}, 2 q$, Cadiz, San Roque, 26.vii. 1986, C. Gielis (CG, RMNH); 1 đ, Málaga, Camino de Ronda, Urb. Madronal, Loma de Colmenas, 3.ix.1988, E. Traugott-Olsen (ZMUC); 20 , Salamanca, San Miguel de Valero $\mathrm{N}, 3 \mathrm{~km}$ S of Linares de Riofrio, 850m, 2.viii.1986, at light, van Nieukerken \& Richter (RMNH). - TURKEY: 10, 3 우, Adana, Taurus, 4 km NE Feke, 1000m, 7.ix.1983, G. Derra; 20, 1 9, Agri, Tahir Gecidi, 2400m, 18.ix.1985, G. Derra (GD, RMNH); 60ิ, Içel [Mersin], Güzeloluk NW Erdemli, 1400m, 16.vii.1986, M. Fibiger (RJ). - UKRAINE: 10 , Crimea, Jalta env., Mys Martyan, 29.viii.1989, Kornilov; 14 ơ, Crimea, Karadag, 20 km W. of Feodosia, 13.vii.1985, 28.vii.1986, 3-21.vii.1987, Budashkin, R. Puplesis (ZIN).

Additional records (A. \& Z. Laštüvka in litt.). - CROATIA: Dalmacia, Oračac and Makarska. - GREECE: $30,3 q$, Seres, Kalokastro, 24.vi.1997; $2 \hat{0}$, 4 + , Akhaia, Kalavrita, 23.vi. 1996. - ITALY, SICILY: Sortino, Solarino, Francavilla, Mandanici, Ficussa. - MACEDONIA: 20ิ, 19 , Negotino, 8.vi. 1997. - SPAIN: 1 온 Málaga, Jimena de Líbar, 27.vi.2003. YUGOSLAVIA: Crna Gora, Budva.

\section{Stigmella karsholti sp. n.}

(figs. 27, 28, 81-84, 115, 116, 141, 142, 160, 169)

Type material. - Holotype $\widehat{0}$ : TUNISIA, Ain Draham area, 5-18.v.1988, Zool. Mus. Copenhagen Exp. [O. Karsholt leg.], genitalia slide EJvN 3148 (ZMUC). Paratypes: $7 \hat{0}, 8 \%$ : TUNISIA: $7 \hat{0}, 6 q$, same data as holotype, genitalia slides ô: EJvN3147, RJ1660, ㅇ: EJvN3157, 3160, 3164, RJ1661, 1772 (ZMUC, RMNH, RJ); 2 ㅇ, 7 km S Ain Draham, Les Chênes, 22.iii.1986, mines on Quercus mirbeckii, genitalia slide 3183 [O. Karsholt leg.] (ZMUC).

\section{Diagnosis}

A large species; male externally superficially resembling S. samiatella or occasionally some other species, but androconials very characteristic. Female resem- 

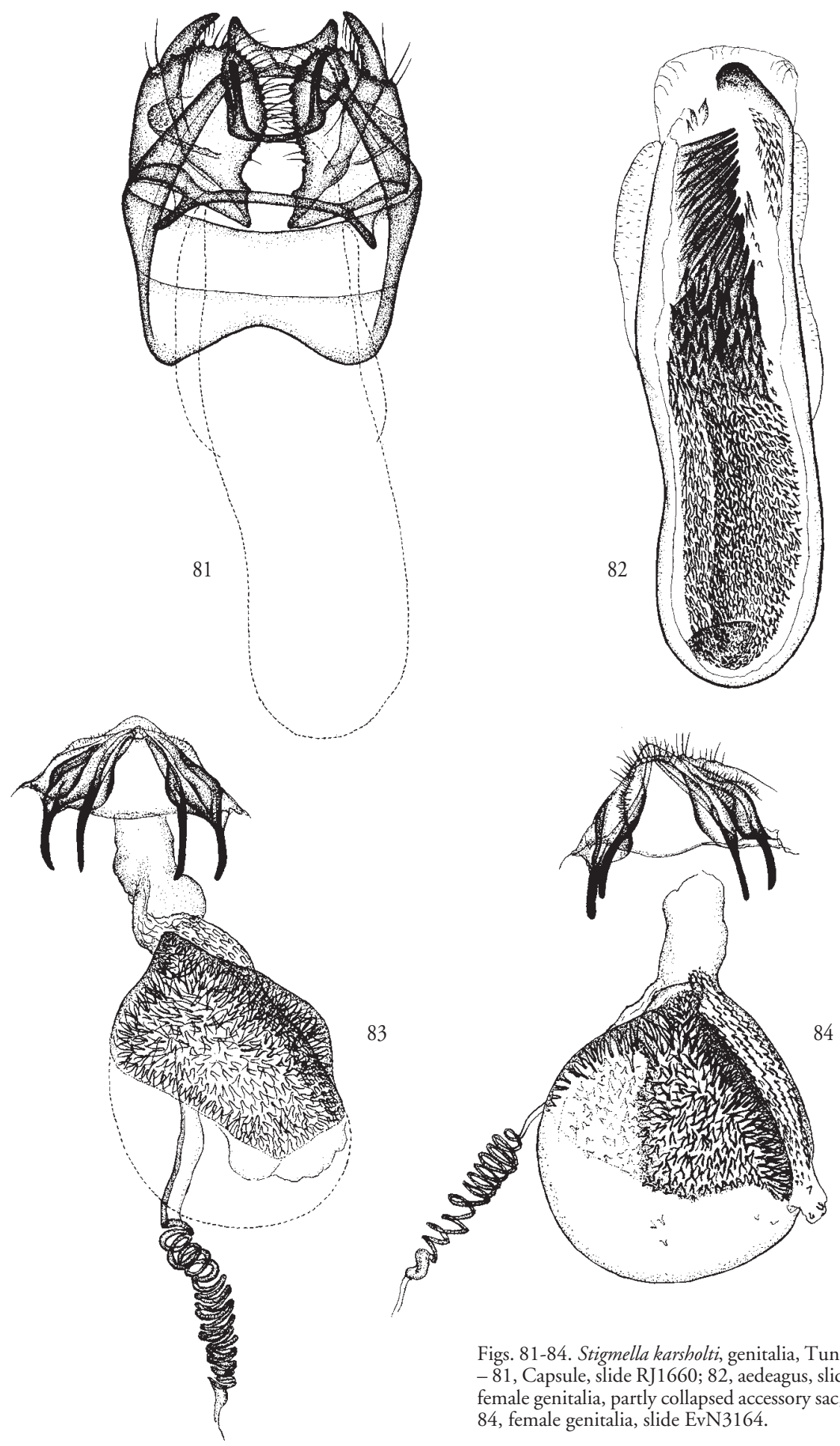

Figs. 81-84. Stigmella karsholti, genitalia, Tunisia, paratypes. - 81, Capsule, slide RJ1660; 82, aedeagus, slide RJ1660; 83, female genitalia, partly collapsed accessory sac, slide RJ1661; 84, female genitalia, slide EvN3164. 
bling other pale headed species, but more coarsely scaled, larger and with more antennal segments than other deciduous oak feeders. Male genitalia characterized by very long aedeagus, only comparable to that of $S$. basiguttella, but even longer, and terminal large spines absent. Female genitalia very characteristic.

\section{Description}

Male (fig. 27). - Forewing length 2.8-3.3 mm, wingspan $6.5-6.8 \mathrm{~mm}$. Head: frontal tuft ochreous, on vertex darker grey-brown; collar almost white. Scape white. Antenna pale grey brown, $2 / 3$ of forewing length, with 37-44 segments. Thorax dark bronze brown. Forewing dark bronze brown, more coarsely scaled and darker towards the tip, some of the scales with strong purplish or bluish iridescence; terminal cilia concolorous; underside bronze-brown, slightly darker along costa; from $1 / 3$ to $2 / 3$ of dorsum a brush of long dark fuscous hair-scales. Hindwing coarsely scaled, grey-brown, dorsum with dark grey-brown androconial scales, extending into fringe to at most half cilia length; in addition dark hair-scales of approximately same length as cilia; along costa two types of spatulate androconial scales: very short ones, shorter than hindwing width and longer ones, approximately as long as dorsal cilia; underside grey brown. Abdomen dark grey brown, with pale yellowish anal tufts inserted on well sclerotized plates.

Female (fig. 28). - Forewing length 2.5-2.9 mm, wingspan 5.7-6.6 $\mathrm{mm}$. Antenna about half forewing length, with 30-33 segments. Head yellow, otherwise as male except for androconial scales.

Male genitalia (figs. 81, 82, 115, 116). - Measurements: see table 3 . Vinculum anteriorly slightly bilobed. Uncus with two, almost triangular, widely separate processes. Gnathos with long, widely separated posterior processes. Valva with pointed distal process about $1 / 3$ valva length; inner margin sinuous, distally with pronounced inner lobe; sublateral processes about $1 / 3$ transtilla length. Aedeagus very long (longest in the group), aedeagal tube with strongly sclerotized distal lobe at right side, resembling large cornutus; vesica with many cornuti, in basal half only small cornuti, in distal half longer needle-shaped cornuti, forming a group at left side, distalmost at right side a group of smaller ones; manica covering distal $2 / 3$ of aedeagus, covered with small spines and pectinations.

Female genitalia (figs. 83, 84, 141, 142, 160). - Bursa reduced, not visible in preparations. Accessory sac strongly sclerotized in distal half, globular (in slides often collapsing), distal $1 / 2$ to $2 / 3$ covered with spines; opening to ductus bursae in anterior part, ductus curving almost $180^{\circ}$; ductus also covered with spines. Ductus spermathecae coiled, with about 11-12 convolutions.

\section{Biology}

Hostplant. - Quercus canariensi** ${ }^{*}=$ Q. mirbeckii).

Leafmine. - Unknown, the mines from which the females were reared, have not been preserved (O. Karsholt, pers. comm.).

Life-history. - The larva was taken in March, all adults in May.

\section{Distribution (fig. 169)}

Only known from northern Tunisia in the extensive oak forests near Ain Draham.

\section{Etymology}

A noun in genitive case, named after its collector Ole Karsholt of the Zoological Museum in Copenhagen. It is a pleasure to name this species after our friend Ole, who has supported both of us in many ways and with whom cooperation is always a pleasure.

\section{Stigmella samiatella (Zeller)}

(figs. 29-31, 85, 86, 117-119, 143, 161, 177)

Lyonetia samiatella Zeller, 1839: 215. Lectotype + : POLAND: [Glogow = Glogau], Zeller Coll., Walsingham Collection 101203, B.M. क genitalia Slide No. 14831 (BMNH) (designated by Johansson 1971: 256) [examined].

Nepticula samiatella; Johansson 1971: 256, Borkowski 1972: 789, Klimesch 1978: 245.

Stigmella samiatella; Klimesch 1951b: 56, fig. 46 (male genitalia), Emmet 1976a: 244, Johansson \& Nielsen 1990: 235, Puplesis 1994: 157, A. \& Z. Laštu̇vka 1997: 112.

\section{Diagnosis}

Male recognised by combination of dark head, uniform brownish forewings and distinctly brown hindwing. S. basiguttella can be very similar when the pale pattern is obsolete, but usually a remnant of the basal spot can be observed and in the middle of the wing, $S$. basiguttella is almost always paler and has always a grey hindwing. S. trojana and bicuspidata superficially resemble samiatella, but have narrow spatulate scales along hindwing dorsum, see also S. karsholti. More problematic are the occasional pale headed $S$. samiatella (fig. 31), which occur more frequently in southern Europe. These are very similar to $S$. roborella and less so to $S$. eberhardi, which usually has darker hindwings.

Females are inseparable from S. atricapitella and can to some extent be confused with S. basiguttella, trojana or zangherii when the forewing is dark.

Male and female genitalia (measurements see table 3) highly characteristic: the male by the low density of cornuti, arranged in three groups, and the female by the long spiny ductus leading to the accessory sac. There is some variability in the female genitalia shown here in figs. 85-86: this is explained partly by the smaller size of some specimens, but the more globular accessory sac is probably also a result of mating, after 

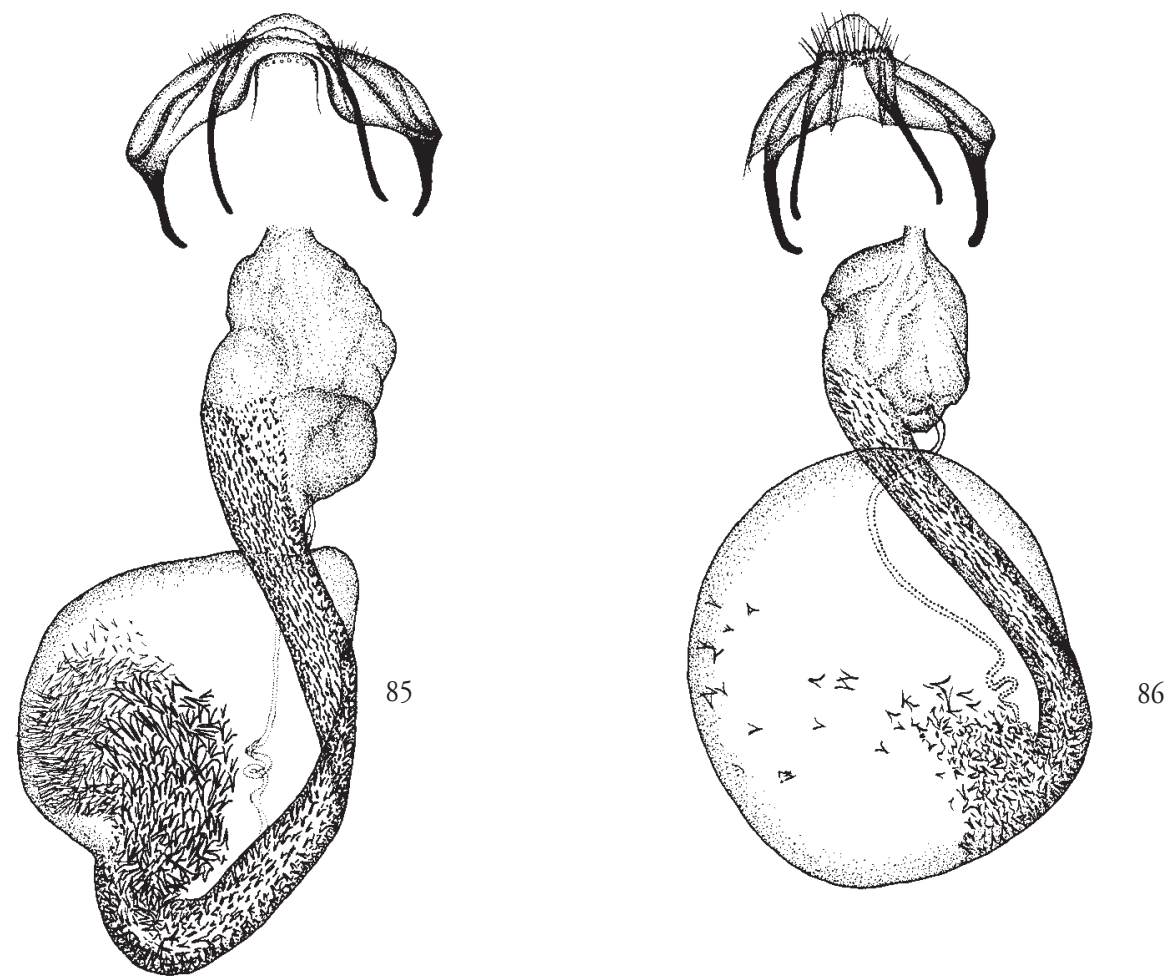

Figs. 85-86. Stigmella samiatella, female genitalia, showing variability. - 85, Sweden, slide RJ2271, 'normal type'; 86, Greece, Kos, slide RJ1729, smaller genitalia, globular bursa.

which the sac is expanded and the spines are spread out. Also the male genitalia show some variation in shape of valva and length of aedeagus.

\section{Biology}

Hostplants. - Castanea sativa*, Quercus ithaburensis subsp. macrolepis (Klimesch 1978), Q. petraea**, Q. pubescens*, Q. pyrenaica*, Q. robur**. S. samiatella is the only Stigmella species that is frequently reared from Castanea, the only other Stigmella known with certainty is $S$. basiguttella.

Leafmine. - A gallery with frass first in narrow midline, later in a broad band. Egg reported on leaf underside (Borkowski 1972) or on leaf upperside (Johansson \& Nielsen 1990), probably a variable character; eggs usually against a vein; in the few mines studied by us, from which adults were reared, the egg indeed was on leaf underside. Larva yellow, no detailed description because of lack of material (Gustafsson \& Van Nieukerken 1990). Mine similar to those of S. ruficapitella, svenssoni and sometimes atricapitella or zangherii.

Life-history. - Bivoltine, larvae in June-early August and September-November, adults April-Septem- ber, October in southern Europe. In Britain believed to be (partly) univoltine (Emmet 1976b), but in the Netherlands and Sweden clearly bivoltine. The species is remarkably frequently taken at light, in the Netherlands most records are from light collected adults, and very few only of reared adults. The reason may be that samiatella prefers leaves higher up in the canopy, or occurs more frequent in periods when there are few other miners, and thus less collecting activity.

\section{Distribution (fig. 177)}

Throughout Europe and Southwest Asia, common almost everywhere. No positive records from: Albania, Belarus, Bosnia, Bulgaria, Ireland, Macedonia, Moldova or Portugal. New (or confirmed) records from Georgia and Russia (also recorded by Shmytova 2002). Highest altitude: $2400 \mathrm{~m}$ in Turkey.

\section{Remarks}

Cronau \& Menken (1990) named one of the species of which they analysed several allozymes on the advice of EvN as S. samiatella. This was based on the assumption that in the Netherlands this species is commoner than $S$. ruficapitella, which makes similar 
mines. However, although this is true when we look at the total amount of specimens taken (see material), it is now our experience that $S$. samiatella is relatively seldom reared from larvae. We now think that this material most likely belonged to $S$. ruficapitella, the more so because B. Gustafsson (in litt.) found that Dutch larvae, tentatively identified as $S$. samiatella, appeared indistinguishable from Swedish ruficapitella.

Material examined. $-237 \hat{\sigma}, 103 q, 6$ adults. - AUSTRIA:

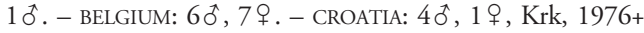
1988, E. Jäckh, G. Baldizzone (USNM, GB, RMNH). - FRANCE:

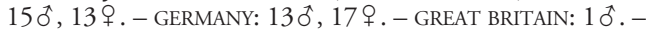
GEORGIA: 20 , Abchazia, Gumista reserve, 9.vii.1978, A. Zagulajev; 30, Lagodekhi, 7+21.vi.1986, 21.vii.1986, A. Lvovskij, S. Seksaeva (ZIN). - GREECE: 1 ㅇ, Kos, Asfendiou, 12.x.1988, R. Johansson (RJ); 10 , Chios, Limnia, 15m, 15.ix.1996, R. Sutter (RS); 10, Evvoia, Dhírfis Oros, S. slopes, 34S GH4876, 700m, 12.ix.1980, Quercus pubescens, e.1.28-30.iii.1981, S.B.J. Menken \& E. J. van Nieukerken (ZMAN); 10, Serrai, Shymon Delta, 16.viii.1985, A. Moberg (NHRS). - ITALY: $80,16 \%$. - LATVIA: $2 \sigma^{\dagger}$. - LITHUANIA: $20^{\circ}$. - NETHERLANDS: $152 \hat{0}, 32$ + . - RUSSIA: $1 \hat{0}$, Belgorod Obl., Borisovska, reserve Les na Worskle, 19.vii.1986, Gusarov (ZIN); 1 के, Uljanovsk, Povolzhye, Shikovka, 200 km S Uljanovsk, 20.vii.1993, V. Zolotuhin (vZ); 2 ㅇ, Volgograd obl., Krasnoarm. r-n, Chapurnikovsk b., 25.vii+21.viii.1999, D. A. Komarov (RMNH, VZ); 1 ô, Volgograd obl., Sredneakhtub r-n, okr. pos. Tumak, 22.vi.1997, D.A. Komarov (vz). - SLOVAKIA: $2 \hat{\sigma}, 1+$. - SPAIN: $3 \hat{\sigma}, 2$, Salamanca, San Miguel de Valero N, $3 \mathrm{~km} \mathrm{~S}$ of Linares de Riofrio, 850m, 2.viii.1986, Quercus pyrenaica forest and heathland; at light, van Nieukerken \& Richter (RMNH); 1 \%, Segovia, Riazza, 3.viii.1986, C. Gielis (CG); 5 ô, Segovia, San Ildelfonso, 26.vi+7.vii.1902, Chrétien (MNHN); $10 \hat{0}, 2$ ㅇ Teruel, Noguera, 1500m, 11.vii.

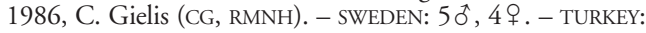
1 , , Agri, Tahir Gecidi, 2400m, 18.ix. 1985, G. Derra (GD); 2 ㅇ, Ankara, 20 km NW Kizilcahamam, 1200m, 24.vii.1986, M. Fibiger (RJ); $1 \delta$, Gümüshane, Pirnakapan, $1800 \mathrm{~m}$, 19.vii.1989, Fibiger \& Esser (ZMUC); 10 , Hakkâri, Tanin Daglari, 3 km E Mutluca, 0.5 km W Baharan Köy, 1250m, 23.iv.1987, H.P. Schreier (GD, RMNH); 1 으, Konya, 5 km SE Ücpinar, road Bozkor-Hadim, Taurus, 1100m, 13.vii. 1986, M. Fibiger (RJ); 10 , Nigde, $40 \mathrm{~km}$ NW Nigde, Sivrihisar pass, 1700m, 4.viii.1989, Fibiger \& Esser (ZMUC). - UKRAINE: $1 \hat{\sigma}$, Borshchiv (former Borszczow), 'Dzwinograd', 9.vi.1937, Toll (ISZP); 20 , Kiev, 6-11.vi.1934, A. Lebedev (ZIN).

Additional records (A. \& Z. Laštuivka, in litt.). - GREECE: 10 , Pieria, Leptokaria, 22.vi.1997; 20 , Ioannina, Kepesovo, 13.vi.1996. - ITALY, SICILY: Francavilla; Etna, Monte Arso. SLOVENIA: Nanos mts., Jan Liška (AL). - SPAIN: 1 ô, Cáceres, Piornal, 1200m, 20.vi.2003; 1 đ̃ Cáceres, San Juan, 400m, 21.vi.2003; 1 ơ, Cuenca, Boniches; 1200m, 17.vi.2002; 3 ô, Gerona, Anglés, 500m, 24.vi.1991.

\section{Stigmella roborella (Johansson)}

(figs. 32, 36, 120-123, 144, 162, 178)

Nepticula roborella Johansson, 1971: 258. Holotype $q$ : SWEDEN: Skåne, Snogeholm, 28.ix.1968, Quercus robur, e.l. 21.iv.1969, R. Johansson, genitalia slide RJ830 (RJ) [examined].
Nepticula roborella; Borkowski 1972: 791.

Stigmella roborella; Emmet 1976a: 241, Johansson \& Nielsen 1990: 236, Puplesis 1994: 158, A. \& Z. Laštüvka 1997: 113.

[Nepticula ruficapitella; Petersen 1930: 46, fig. 6 (male genitalia). Misidentification.]

[Stigmella ruficapitella; Vári 1950: 183, figs. 2, 3, 5 (male, female genitalia, mine), Klimesch 1951b: 56, fig. 47 (male genitalia). Misidentifications.]

\section{Diagnosis}

Male $S$. roborella can be recognised by the combination of a pale head, uniform forewings, and hindwings with a not very distinct cover of pale brown androconial scales. S. eberhardi has usually much darker (bronze brown) androconials, and S. svenssoni and szoecsiella have grey hindwings without androconials. Moreover, S. svenssoni has special scaling on the forewing underside. The rare pale-headed form of S. samiatella is particularly confusing, since it resembles $S$. roborella closely. Females resemble all the other pale-headed species, but $S$. roborella is the only one with a more or less pointed abdominal tip (ovipositor); it has more antennal segments (28-31) than S. ruficapitella, dorsiguttella or szoecsiella (23-27) and fewer than S. suberivora or ilicifoliella (33-40). In southern localities, especially Sicily (where S. eberhardi has not yet been found), the androconial scales may be as dark as in eberhardi, making it impossible to separate the species on externals.

Male genitalia (measurements see table 3) are characterized by a pointed inner lobe of the valva, resulting in a valva with two pointed tips, and the slightly curved vesica with three groups of cornuti, although less distinct than in the similar S. eberhardi. The vinculum is very variable in the length of the ventral plate (see figs. 120, 122).

Female genitalia characterised by the single coil and the spines in posterior part.

\section{Biology}

Hostplants. - Quercus cerris (Szőcs 1977), Q. pe-

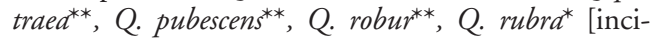
dentally].

Leafmine. - A long gallery, often with straight parts, with frass usually in a narrow line throughout, occasionally slightly widened in second part. Egg on either side of the leaf, although Borkowski (1972) only mentioned upperside; usually against a vein. Larva yellow, pale headed (see photograph in Huisman et al. 2001). Mine similar to that of S. eberhardi, atricapitella and dorsiguttella.

Life-history. - Bivoltine, larvae in June-July and September-November, adults from April-September, usually somewhat later than $S$. ruficapitella.

\section{Distribution (fig.178)}

Throughout Europe, also in Southwest Asia, com- 

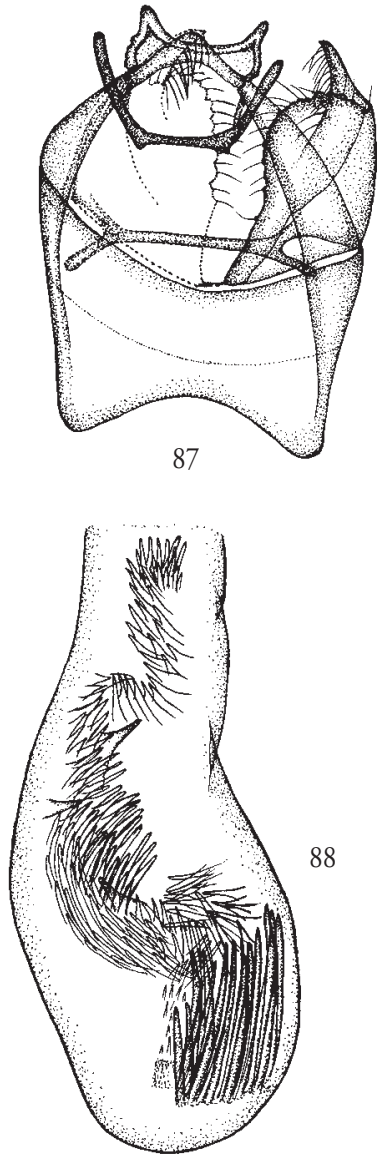
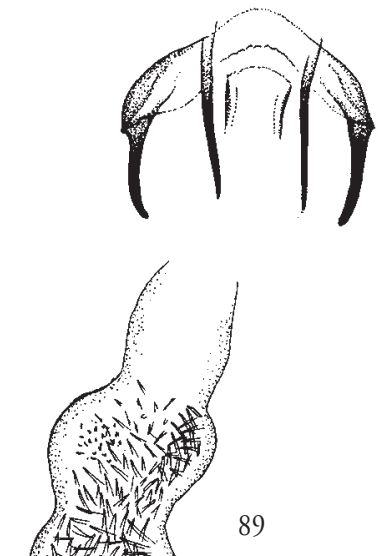

89

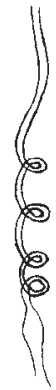

Figs. 87-89. Stigmella eberhardi, genitalia, Italy, paratypes. - 87, 88, male genitalia, slide EJ5623; 89, female genitalia, slide RJ948, inset: ductus spermathecae from slide EJ5073.

mon almost everywhere, but fewer records in the Mediterranean countries. Since its description in 1971, positively recorded from most European countries (Borkowski 1972, Budashkin 1988, Gielis \& Hull 1975, Johansson 1971, Johansson \& Nielsen 1990, Karsholt \& Nielsen 1976, A. \& Z. Laštüvka 1990, 1991, 1997, Z. \& A. Laštüvka 1998, Lo Duca et al. 2002, Puplesis 1994, Sulcs \& Sulcs 1989, Szőcs 1977), except Albania, Belarus, Bosnia, Bulgaria, Ireland, Moldova, Portugal, Romania or Yugoslavia. New records for Georgia, Macedonia and Turkey. One of the few species on Mediterranean islands: Sardinia and Sicily. Highest altitude: $1500 \mathrm{~m}$ in Spain.

Material examined. - $168 \hat{\sigma}, 1269,7$ adults. - AUSTRIA:

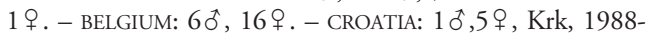
1990, G. Baldizzone (GB, RMNH). - FRANCE: 18 $\sigma^{\star}, 11$ ㅇ. GEORGIA: 20ิ, Abchazia, Sukhumi, 27.vi.1989, R. Puplesis
(ZIN, RMNH). - GERMANY: $14 \hat{0}, 11$ + - GREAT BRITAIN: $4 \hat{0}$, 3․ - GREECE: 1 ô, Korfu: Benitses, 25-26.iv.1977, V. Varis; 10, Larissa, Olympos, 700-2100m, 21-26.v.1990, O. Karsholt, (ZMUC); 1 \% , Makedonia, Leptokaria, $15 \mathrm{~km} \mathrm{~W}$ Olympos, 750m, 21-23.v.2001, J. Junnilainen (JJ); 1 ô, Zakynthos, S Vasilikos, 20m, 13.ix.1997, R. Sutter (RS). - ITALY: 7 đ, 15 ․ - ITALY, SARDINIA: 1 , Nuoro Belvi, Quercus pubescens, e.1. 19.iv.1976 F. Hartig; 19, ibid., 17.vii.1975, F. Hartig (MRSN); 19 , Nuoro, Funtana Raminosa, 900m, 4.viii.1984, J. H. Kuchlein (JHK); 1 \&, Nuoro, Gennargentu, 5.ix.1974, F. Hartig (MRSN); 1 ㅇ, Nuoro, Mamoiada, 650m, 25.vii.1983, J. H. Kuchlein (JHK). - ITALY, SICILY: 10 , Palermo, Cefalù, $500 \mathrm{~m}$, 9.vii. 2000 , P. Triberti (PT). - LATVIA: 3 ㅇ․ - NETHERLANDS: 79 0 , 549,7 adults. - RUSSIA: $1 \hat{0}$, Kalinigrad Obl., Slavsk, 10.ix.1989, R. Puplesis (ZIN); 1 के, Uljanovsk, Vjazovka, 160 km S Uljanovsk, 15-16.vii.1999, A. \& V. Isajevy (VZ). - SPAIN: 20, Teruel, Noguera, 1500m, 11. vii.1986, C. Gielis (CG, RMNH). - SWEDEN: 10 holotype plus an unspecified number. - TURKEY: $1+$, Ankara, Kizilcahamam, 31.vii-1.viii.1963, E. Arenberger (SMNK). - UKRAINE: 1ิे, Kiev, 2.vi.1934, Lebedev; 30 , Crimea Jalta env., Mys 
Martyan, 29.viii.1989, Kornilov; 240َ, Crimea, Kara-Dag, $20 \mathrm{~km}$ W. of Feodosia, 25.vi.1977, 13.v.1985, 29.iv.19869.ix.1986, 5-21.vii.1987, Yu. Budashkin, R. Puplesis, Falkovitsh (ZIN, RMNH.).

Additional records (A. \& Z L Laštüvka in litt.). - GREECE: 10, Messinia, Pirgos, 13.vi.1998. - ITALY, SICILY: Etna, Monte Arso; Ficussa; Francavilla; Mandanici; Solarino; Sortino. - MACEDONIA: Trojáci. - SPAIN: 10 , Cádiz, Alcalá del Valle, 600m, 11.vii.1993; 1 ㅇ, Cuenca, Boniches, 1200m, 17.vi.2002; 1 ㅇ, Toledo, Robledo del Buey, 800m, 18.vi. 2002.

\section{Stigmella eberhardi (Johansson)}

(figs. 33, 34, 87-89, 126, 127, 145, 163, 179)

Nepticula eberhardi Johansson, 1971: 258. Holotype $q$, ITALY: Liguria, Val Merula di Moleto, 13.viii.1968, E. Jäckh, genitalia slide EJ 5073 (USNM) [examined].

Nepticula eberhardi; Borkowski 1972: 794.

Stigmella eberhardi; Van Nieukerken 1986b: 14, A. \& Z. Laštüvka 1990: 187, A. \& Z. Laštuivka 1997: 114.

\section{Diagnosis}

Males usually easy to recognise by the pale head and dark bronzy brown androconials; faded specimens may somewhat resemble $S$. roborella or pale headed $S$. samiatella (particularly in Spain). Sicilian S. roborella has androconials very similar to those of eberhardi. $S$. dorsiguttella has paler brown or orange-yellow androconials and also a clear androconial patch on basal $2 / 3$ of forewing underside. Female very similar to $S$. roborella, but ovipositor not pointed.

Male genitalia resemble $S$. roborella, but valva with rounded inner lobe and aedeagus with broader anterior part and more clearly contorted vesica. Female genitalia with accessory sac very distinctly contorted, unmistakeable.

\section{Description}

Male (fig. 33). - Forewing length 2.3-3.1 mm, wingspan $5.0-6.8 \mathrm{~mm}$. Frontal tuft yellowish white, ochreous or occasionally mixed white and brown, scape and collar white, antenna with 33-40 segments. Thorax and forewings dark bronze brown with faint bronze reflections; underside bluish black. Hindwing covered with almost bluish black androconial scales; underside bluish black. Abdomen brown, anal tufts yellowish, inserted on well sclerotized plates.

Female (fig. 34). - Forewing length 2.1-2.6 mm; wingspan 4.9-5.6 mm. Antenna with 25-31 segments. As male, but hindwings and underside of wings grey; abdomen without pointed tip.

Male genitalia (figs. 87, 88, 124, 125). - Measurements: see table 3 . Vinculum anteriorly slightly bilobed. Uncus with triangular, widely separated horns. Gnathos with long and thin, widely separate processes. Valva with pointed distal process of about $1 / 3$ valval length, inner lobe gradually rounded; sublateral processes about $1 / 3$ transtilla length. Aedeagus long, basally widened; vesica coiled, forming almost a complete coil, with several groups of cornuti, anteriorly a group of long ones, more posteriorly the cornuti becoming shorter; manica not very conspicuous, only covering distalmost part of aedeagus.

Female genitalia (figs. 89, 145, 163). - Accessory sac distinctly coiled, forming up to four coils, covered with many spines, except in anteriormost part. Ductus spermathecae with about 5 convolutions.

\section{Biology}

Hostplants. - Quercus ilex**, Q. petraed**, Q. pu-

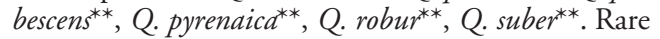
on the evergreen oaks ( $Q$. ilex and suber), and only on teneral leaves of these.

Leafmine. - A gallery with thin frass throughout. Egg usually on leaf upperside. Larva yellow. Mine almost indistinguishable from that of $S$. atricapitella or roborella.

Life-history. - Bivoltine, or possibly continuously brooded in south, larvae found in February (Andalusia: on Q. suber) and late May to December (on deciduous oaks), adults taken in April-September.

\section{Distribution (fig. 179)}

A southern species, widespread in Mediterranean region and reaching southern Central Europe. Positively recorded from Czech Republic and Slovakia (A. \& Z. Laštuivka 1990), Austria (Kasy 1979), Hungary, southeastern France, Spain (Vives Moreno 1991), Portugal, Italy, Sardinia, Slovenia (new record), Croatia (new record), Macedonia (new record), Greece (Van Nieukerken 1996), Turkey (new record). The record from northern France (Gibeaux 1999) is not accepted, as it may have been $S$. samiatella. Surprisingly as yet not recorded from Sicily, where it seems to be replaced by a form of $S$. roborella with hindwings as in eberhardi. Not yet found in North Africa. Highest altitude: 1500 $\mathrm{m}$ in Turkey.

Material examined. $-35 \hat{\sigma}, 38 ․$. AUSTRIA: 2 , Niederösterreich, Glaslauterriegel, Gümpoldskirchen, 10.viii.1983, F. Kasy (NMW, RMNH); 2 \% , ibidem, 300-340m, 1.x.1983, Quercus pubescens, e.l. 17-18.iv.1984, E. J. van Nieukerken $\&$ J.J. Boomsma; 2 , N Niederösterreich, Hainburg: Hundsheimer Berg, 200-400m, 23.x.1983, Quercus pubescens, e.l. 14-18.iv.1984, E. J. van Nieukerken (RMNH); 10 , 1 ㅇ, ibidem, 14.viii.1979, F. Kasy (NMW). - CROATIA: 10, Krk, Misucaynica, 9.vii.1976, E. Jäckh (USNM); 1 + , Krk, Picik, 19.vii.2001, G. Baldizzone (GB). - FRANCE: 40, 2 으, Alpes Maritimes, [Cannes], Quercus pubescens e.l. [A. Constant] [partly labelled: 'sp.n. Q. pubescens']; 10 , ibid., Quercus ilex e.l. (MNHN); 20 , Var, Nans-les-Pins, Quercus pubescens, e.l. 15-20.iv.1983, R. Buvat; 1 , Vaucluse, Viens, 19.iv.1976, R. Buvat (MHNM). - GREECE: 10, 2 으, Evro, $35 \mathrm{~km} \mathrm{~N}$ Alexandropolis, Kirki, 500m, 20-21.viii.1985, A. Moberg, M. Fibiger (NRMS, ZMUC); 1 tิ, 1 으, Evro, Kavisos, 100m, 
22-23.viii.1985, A. Moberg (NRMS). - HUNGARY: 1 o, Budaörs, 23.vi.1971, Quercus petraea, J. Szőcs (MCNT). - ITALY: 19 , Latina, Monti Aurunci, $4 \mathrm{~km}$ NW Castelforte, $400 \mathrm{~m}$, 22-23.vi.1969, R. Johansson (RJ); 20ิ, 1 올. Potenza, Mte Vulture, Laghi di Monticchio, 750m, 22.xi, 18.xii.1966, Quercus robur, e.l. 25.iii+12.iv.1967, F. Hartig (MRSN); 1 o, Savona, Andora, Conna, 250m, 14.vii.1969, E. Jäckh (RJ); 1 으, ibidem, 300m, 8.ix.1973, G. Baldizzone (GB). - ITALY, SARDINIA: 10 , Nuoro, Mt. Istiddi, 700m, 30.vi.1976, G. Derra (GD); $1 \hat{0}$, Nuoro, Belvi, Mt. Istiddi, $700 \mathrm{~m}$, 30.viii.1974, F. Hartig; 1 \% , ibidem, 900m, 9.vi.1977, F. Hartig; 10 , 2 \% , Nuoro, Belvi, Quercus pubescens, e.l. 1920.iv.1976, F. Hartig; 2 q , Nuoro, Belvi, surroundings, 700m, 17.vii.1975, F. Hartig (MRSN); 10 , Nuoro, Funtana Raminosa, 900m, 4.viii.1984, J. H. Kuchlein (JHK); 1 के, Nuoro, Gennargentu, Arcu Tascussi, 1000m, 28.vii.1981, G. Baldizzone (GB); 1 ô, Nuoro, Mamoiada, 31.vii.1983, J. H. Kuchlein (JHK). - PORTUGAL: 1 , , Beira Alta, Videmonte, 750m, 5.ix.2001, M. F. V. Corley (MC); $1 \hat{\jmath}, 2$, [Beira Baixa, Sao Fiel], [e.l.] 8.v., Quercus pyrenaica [as Q. toza], [Mendes], coll. Joannis (MNHN). - SLOVAKIA: 2q, Slov. Kras.: Háj, 9 km W Moldava n. B., 200m, 9.x.1992, leafmines, Quercus pubescens, e.l. 23-24.iii.1993, E. J. van Nieukerken (RMNH). - SPAIN: 10 , 1 \% , Barcelona, La Pobla de Claramunt, Anoia, 26.viii.2000, E. Requena (RMNH); 1 के, 1 ․ Cadiz, San Roque, 26.vii.1986, C. Gielis (CG); 10 , Cadiz, Sierra de Ojén, 200m, 28.viii.1982, A. Vives Moreno (AV); 20 , 1 으, Cuenca, Cuenca, 900m, 16.vii.1986, C. Gielis (CG, RMNH); $1 \hat{\delta}$, Cuenca, Sa de Altomira, Vellisca, 1000m, 11.viii.1983, A. Vives Moreno (AV); 1 ô, Málaga, 6 km E of Marbella: Los Monteros, 8.ii.1984, Quercus suber, e.l. 2.iii.1984, E. J. van Nieukerken (RMNH); 10 đ , 2 ㅇ , Málaga, Camino de Istan, 400m, 28.vi+21.vii.1972, 4.vii.1973, E. Traugott-Olsen (RMNH, ZMUC); 1 đิ, Málaga, Camino de Istan, 200m, 18.vi.1983, E. Traugott-Olsen (ZMUC); $2 \hat{o}$, 3 ㅇ. Málaga, Camino de Ronda, Urb. Madronal, Loma de Colmenas, 14.viii.1988, 3.ix.1988, E. Traugott-Olsen (ZMUC, RMNH). - TURKEY: 1 ô, Isparta, Aksehir $30 \mathrm{~km} \mathrm{SW,}$ Sultan Daglari, 1500m, 31.viii.1997, K. Nupponen \& J. Junnilainen; 10 , Isparta, Aksehir, $35 \mathrm{~km}$ SW: Cetince, 1200m, 9-13.v.2000, J. Junnilainen; 1 ㅇ , Konya, Konya, 30 km W, 21.v.1997, K. Nupponen \& J. Junnilainen (J); 2 옹, Konya, Eregli, Bolkar Daglari, 1200m, 24.vi.1968, M. \& W. Glaser (SMNK).

Additional records (A. \& Z . Laštüvka, in litt.). - CROATIA: Istra, Rabac; Dalmacia, Oračac. - GREECE: 20 , Preveza, Thesprotiko, 11.vi.1997; 30ิ, 1 q , Messinia, Kardamili, 20.vi. 1996; 10, Akhaia, Kalavrita, 20.vi.1997. - ITALY: Campania, San Salvatore Telesino. - MACEDONIA: Negotino. - SLOVEnia: Nanos mts. - SPAIN: 19 , Cáceres, Piornal, $1200 \mathrm{~m}, 20 . v i .2003 ; 1 \%$, Huelva, Bonares, $100 \mathrm{~m}$, 26.vi.2003.

\section{DisCUSSION}

\section{Phylogenetic remarks}

A phylogenetic analysis was not part of this study. Such an analysis should also include species other than the oak feeders and preferably also the Eastern Palearctic species. Moreover, a framework of the phylogeny of the genus Stigmella is still lacking; we expect that on the basis of ongoing DNA studies this can hopefully be prepared in the coming years.

There is no clear support yet for the monophyly of the ruficapitella group, but certain parts of it most likely form monophyletic entities. A few characters apparently showing phylogenetic signal are given below:

1. Female tergum 8 with two parallel furrows and ridges. This character may well be a synapomorphy for most of this group: it is only absent from $S$. fasciata, some Eastern Palaearctic species and the nonQuercus feeders.

2. Development of accessory sac in female. In the group of suberivora and related species (here species 1 to 5) and several East-Palearctic species, the accessory sac is well developed and folded, but not spiny. In these species the bursa itself is still well developed. This condition resembles many other Stigmella species and is therefore regarded as the plesiomorphic condition.

In the other species treated here and S. tristis, plus several Eastern Palearctic species, the accessory sac is well developed and usually contains a large number of spines and some other sclerotizations, whereas the bursa proper is either lost or very much reduced (Van Nieukerken 1986a).

The bursa of the non-Quercus feeders $S$. hemargyrella and $S$. speciosa is rather atypical, that of $S$. lonicerarum belongs to the suberivora type.

3. Spatulate androconial scales on male hindwing. This character occurs scattered in the group. All the species related to S. suberivora (2-5), S. ruficapitella and $S$. atricapitella show it very convincingly, $S$. trojana and S. bicuspidata have few such scales. Also various Eastern Palearctic species show this character, whereas it is rare elsewhere in Stigmella (i.e. in S. lemniscella (Zeller)). It may therefore well be an apomorphy for the group, which is lost again several times.

4. Abdominal tufts on segment 8 in male inserted on well sclerotized plates. This occurs in most species, except $S$. fasciata.

5. Widening of aedeagus and coiling of vesica. This distinct character is shared by species 9-13 as a possible synapomorphy. It somewhat resembles the more elaborate coiling of the vesica in the species of the castanopsiella subgroup (Van Nieukerken \& Liu 2000), although these species differ widely in other characters.

\section{Biogeographic considerations}

The distribution pattern of the oak feeders in the Western Palearctic is becoming better known, although we still have very little knowledge of northern Africa, the Levantine coast, the Caucasus and the mountain oak forests of Iran. Also in the central part of the Balkan Peninsula, the Ukraine and Russia data are scarce, but new data there will probably mostly serve to fill in the gaps in known distributions.

Seven species are very widespread, with $S$. svenssoni 
and ruficapitella showing a more northern distribution, S. atricapitella a more southwestern and $S$. dorsiguttella a more southeastern one. Of the remaining species, two are widespread in southern and southern central Europe: S. zangherii and S. eberhardi, only three are confined to the southwest $(S$. suberivora, ilicifoliella and karsholti) and the remaining six to the southeast of Europe and Asia Minor. It is not surprising that Turkey has the richest fauna with thirteen known species, which probably will increase after more focussed collecting: Turkey also has the highest number of oak species recorded. Also Greece and Italy have rich faunas with twelve and eleven species respectively. The western European fauna is much poorer, with only eight species recorded from Spain.

Also remarkable is the poor fauna of the larger Mediterranean islands, with the exception of Sicily, which still has five species, probably because it is close to mainland Italy. From the other islands Sardinia has been sampled best: only $S$. suberivora, eberhardi and roborella are recorded here, and on Crete only $S$. cocciferae. No species are yet known from Cyprus, despite the presence of forests of Quercus alnifolia Poech, which harbour at least two Ectoedemia species (Van Nieukerken 1985).

\section{ACKNOWLEDGEMENTS}

We are particularly grateful to Aleš and Zdeněk Laštuivka for the stream of unpublished information, gifts of material and comments on an earlier version. This paper would not have been possible without all the material we have been able to borrow and information we got from: Giorgio Baldizzone (Asti, Italy), Kees van den Berg (Hoogland, Netherlands), W. Biesenbaum (Velbert-Langenberg, Germany), Kenneth G. M. Bond (Cork, Ireland), the late R. Buvat (Marseille, France), Achille Casale (Torino, Italy), Martin F. V. Corley (Faringdon, Great Britain), Don R. Davis (Washington, USA), Gg. Derra (Bamberg, Germany), Cees Gielis (Lexmond, Netherlands), Bert Gustafsson (Stockholm, Sweden), Peter Huemer (Innsbruck, Austria), Jari Junnilainen (Vantaa, Finland), Ole Karsholt (Copenhagen, Denmark), the late F. Kasy (Vienna, Austria), Sjaak Koster (Callantsoog, Netherlands), Joop Kuchlein (Wageningen, Netherlands), András Kún (Budapest, Hungary), John Langmaid (Southsea, Great Britain), Aleš Laštuivka (Prostejov, Czech Republic), Zdeněk Laštúvka (Brno, Czech Republic), Patrice Leraut (Paris, France), P. Limbourg (Brussels, Belgium), Gerard Luquet (Paris, France), Joël Minet (Paris, France), Bruno Massa (Palermo, Italy), Rimantas Puplesis (Vilnius, Lithuania), Emili Requeña (Anoia, Spain), Andreas Segerer (München, Germany), H. Steuer (Bad Blankenburg, Germany), R. Sutter (Bitterfeld, Germany), Zdenko Tokár
(Michalovce, Slovakia), Ernst Traugott-Olsen (Marbella, Spain), Paolo Triberti (Verona, Italy), Kevin Tuck (London, Great Britain), Antonio VivesMoreno (Madrid, Spain), Steven Whitebread (Boston, USA), Hugo W. van der Wolf (Nuenen, Netherlands) and Vadim Zolotuhin (Uljanovsk, Russia). Ernestino Maravalhas (Porto, Portugal) helped us in the translation of Mendes' description and provided information on the Mendes collection. The basis for this paper was laid long ago in the nepticulid working group of the Free University of Amsterdam. We would like to acknowledge Ria Dekker (Amsterdam), who as a student of EvN did pioneer research on the Dutch species of this group back in 1979-1981 and EvN's former colleagues Georgina Bryan, Koos Boomsma and Steph Menken for stimulating co-operation. Robert Hoare (Auckland, New Zealand) is acknowledged for his constructive remarks on an earlier version. Staff of the production department of Naturalis is acknowledged for adequate and professional assistance with the preparation of this publication.

\section{REFERENCES}

Agassiz, D. J. L., 1988. Microlepidoptera - a review of the year 1986. - Entomologist's Record and Journal of Variation 100 (5-6): 118-130.

Agenjo, R., 1964. Catálogo ordenador de los lepidópteros en España. - Graellsia 20 (4-6): appendix [1-12].

Aguiar, A. M. F. \& O. Karsholt, in press. Lepidoptera. Systematic catalogue of the Madeira Archipelago and Selvages Islands. - Boletim do Museo Municipal do Funchal. Supplement.

Amaral Franco, J. d., 1990. Quercus L. - In: S. Castroviejo, M. Laínz, G. López González, P. Montserrat, F. Muñoz Garnendia, J. Paiva \& L. Villar (eds.), Flora Iberica. Plantes vasculares de la Península Ibérica e Islas Baleares, 2. Platanaceae-Plumbaginaceae (partim). 2: 15-36. Real Jardín Botánico, Madrid.

Amsel, H. G. \& M. Hering, 1931. Beitrag zur Kenntnis der Minenfauna Palästinas. - Deutsche Entomologische Zeitschrift 1931: 113-152.

Beirne, P. B., 1945. The male genitalia of the British Stigmellidae (Nepticulidae) (Lep.). - Proceedings of the Royal Irish Academy. Section B 50: 191-218.

Borkowski, A., 1972. Studien an Nepticuliden (Lepidoptera). Teil V. Die europäischen Arten der Gattung Nepticula Heyden von Eichen. - Polskie Pismo Entomologiczne 42 (4): 767-799.

Boursin, C., 1936. Note au sujet de la collection de Macrolépidoptères de A. Constant. - Bulletin de la Société Entomologique de France 1936: 143-144.

Budashkin, Y. I., 1988. Nasekomye: Cheshuekrylye. (Insecta: Lepidoptera). - In: Karadagskij Gosudarstvennyj Zapovednik AN Ukrainy. Letopis Prirody. 5: 64-69. Akademiya Nauk Ukrainy, Institut Biologii Yuzhnykh Morej Im. A. O. Kovalevskogo, Karadagskij Filial, Simferopol.

Buhl, O., O. Karsholt, K. Larsen, G. Pallesen, E. Palm \& K. Schnack, 1981. Fund af småsommerfugle fra Danmark i 1979 (Lepidoptera). - Entomologiske Meddelelser 49: 119-136. 
Buhr, H., 1940. Über Verbreitung und ausländische Nahrungspflanzen von Neptikeln. - Zeitschrift des Wiener Entomologen-Vereins 25: 193-196, 209-211, 226-235.

Buszko, J., 1987. Studies on the mining Lepidoptera of Poland, 2. New records of some rare species. - Polskie Pismo Entomologiczne 37: 631-643.

Cronau, J. P. \& S. B. J. Menken, 1990. Biochemical systematics of the leaf mining moth family Nepticulidae (Lepidoptera). 2. Allozyme variability in the Stigmella ruficapitella group. - Netherlands Journal of Zoology 40 (3): 499-513.

Dickerson, B., R. Edmunds, M. Ellis, I. Kimber, D. Manning, B. Pitkin \& P. Talbot, 2003. British leafmining fauna. - http://www.leafmines.co.uk/index.htm [visited on: 21 June 2003].

Diškus, A., 2003. Revizuota Lietuvos Nepticulidae fauna. (Revised fauna of the Nepticulidae of Lithuania). - In: R. Puplesis \& A. Diškus (eds.), The Nepticuloidea \& Tischerioidea (Lepidoptera) - a global review, with strategic regional revisions: 290-317. Lutute publishers, Kaunas.

Diškus, A. \& R. Puplesis, 2003. Catalogue of the world Nepticuloidea \& Tischerioidea. - In: R. Puplesis \& A. Diškus (eds.), The Nepticuloidea \& Tischerioidea (Lepidoptera) - a global review, with strategic regional revisions: 318-436. Lutute publishers, Kaunas.

Draghia, I., 1975. Insecte minere. - In: M. Ionescu (ed.), Grupul de Cercetari complese 'Portile de Fier', seria Monografica. Fauna. Grupul de Cercetari complese 'Portile de Fier', seria Monografica: 255-262. Editura Academiei Republicii Socialiste Romania, Bucuresti.

Dufrane, A., 1955. Microlépidoptères de la Faune paléarctique. - Mémoires de la Société Royale Entomologique de Belgique 27: 184-193.

Ellis, W. N., 2003. De bladmineerders van Nederland. . http://www.xs4all.nl/ - wnellis/index.htm [visited on: 26 March 2003].

Emmet, A. M., 1976a. Nepticulidae. - In: J. Heath (ed.), The moths and butterflies of Great Britain and Ireland, 1. Micropterigidae - Heliozelidae. The moths and butterflies of Great Britain and Ireland 1: 171-267, illustr. Blackwell Scientific Publications Ltd, Oxford \& London.

Emmet, A. M., 1976b. The status in Britain of Stigmella samiatella (Zeller, 1839) and S. svenssoni (Johansson, 1971) (Lep., Nepticulidae). - Entomologist's Record and Journal of Variation 88: 315-318.

Emmet, A. M., 1981. Records of Nepticulidae in Ireland since 1975. - Irish Naturalists' Journal 20 (5): 186-188.

Emmet, A. M., 1985. First and second supplements to the butterflies and moths of the Isle of Man. Second supplement. - Entomologist's Record and Journal of Variation 97 (3-4): (9)-(12), (13)-(16).

Emmet, A. M., 1988. Nepticulidae. - In: A. M. Emmet (ed.), A field guide to the smaller British Lepidoptera. Second edition, revised and enlarged: 17-31. The British Entomological \& Natural History Society, London.

Fischer, M., 1991. Oberrat Dr. Friedrich Kasy zum Gedenken. - Annalen des Naturhistorischen Museums in Wien 92B: 289-290, pl 1.

Gibeaux, C. A., 1999. Liste-inventaire des Lépidoptères du massif de Fontainebleau (Insecta, Lepidoptera). - Bulletin de l' Association des Naturalistes de la Vallée du Loing et du Massif de Fontainebleau 75 (2): 1-64.

Gielis, C. \& M. Hull, 1975. Vlindervangsten in de Eendekooi te Vlaardingen. - Entomologische Berichten, Amsterdam 35 (9): 125-133.
Govaerts, R. \& D. G. Frodin, 1998. World checklist and bibliography of Fagales (Betulaceae, Corylaceae, Fagaceae and Ticodendraceae). - The Royal Botanic Gardens, Kew. 407 pp.

Gustafsson, B., 2003. Svenska fjärilar. - Naturhistoriska Riksmuseet, Stockholm. http://www.nrm.se/en/svenska_fjarilar/svenska_fjarilar.html [visited on: 8 July 2003].

Gustafsson, B. \& E. J. van Nieukerken, 1990. Larvae of Nepticulidae. - In: R. Johansson, E. S. Nielsen, E. J. van Nieukerken \& B. Gustafsson (eds.), The Nepticulidae and Opostegidae (Lepidoptera) of NW Europe. Fauna Entomologica Scandinavica 23: 323-356. E. J. Brill, Leiden.

Haworth, A. H., 1828. Lepidoptera Britannica; sistens digestionem novam Insectorum Lepidopterorum quae in Magna Britannia reperiuntur, larvarum pabulo, temporeque pascendi; expansione alarum; mensibusque volandi; synonymis atque locis observationibusque variis. 4. - J. Murray, London. pp. 513-609.

Heinemann, H. v., 1862. Einige Bemerkungen über die Arten der Gattung Nepticula. - Wiener Entomologische Monatschrift 6 (8): 237-268.

Hering, M., 1957. Bestimmungstabellen der Blattminnen von Europa, 3 vols. - Junk, s-Gravenhage. 1185 pp.

Herrich-Schäffer, G. A. W., 1847-1855. Systematische Bearbeitung der Schmetterlinge von Europa, zugleich als Text, Revision und Supplement zu Jakob Hübner's Sammlung europäischer Schmetterlinge, 5, Die Schaben und Federmotten. - G. J. Manz, Regensburg. 394 pp., plates Tineides 1-124, Pterophorides 1-7, Micropteryges 1.

Huisman, K. J., J. C. Koster, E. J. van Nieukerken \& S. A. Ulenberg, 2001. Nieuwe en interessante Microlepidoptera uit Nederland in het jaar 1999 (Lepidoptera). - Entomologische Berichten, Amsterdam 61 (12): 169-199.

IGN, 1997-2003. Quelles sont les coordonnées géographiques de votre commune ? - Institut Géographique National. http://www.ign.fr/ [visited on: 8 July 2003].

Jalas, J. \& J. Suominen (eds.), 1976. Atlas florae europaeae: distribution of vascular plants in Europe, 3. Salicaceae to Balanophoraceae. - Committee for Mapping the Flora of Europe, Helsinki. 128 pp.

Joannis, J. d., 1909. Contribution a l'étude des Lépidoptères du Morbihan. - Annales de la Société Entomologique de France 77: 688-838, pl. 15, map.

Johansson, R., 1971. Notes on the Nepticulidae (Lepidoptera) I. A revision of the Nepticula ruficapitella group. - Entomologica Scandinavica 2: 241-262.

Johansson, R. \& E. S. Nielsen, 1990. Tribus Nepticulini. In: R. Johansson, E. S. Nielsen, E. J. van Nieukerken \& B. Gustafsson (eds.), The Nepticulidae and Opostegidae (Lepidoptera) of NW Europe. Fauna Entomologica Scandinavica 23: 111-238. E. J. Brill, Leiden etc.

Jürivete, U., J. Kaitila, T. Kesküla, K. Nupponen, J. Viidalepp \& E. Ounap, 2000. Estonian Lepidoptera, catalogue. - Estonian Lepidopterists' Society, Tallinn. 150 pp.

Karsholt, O. \& E. S. Nielsen, 1976. Systematisk fortegnelse over Danmarks sommerfugle. - Scandinavian Science press, Klampenborg. 128 pp.

Kasy, F., 1965. Österreichische entomologische Expeditionen nach Persien und Afghanistan, Bericht über die Expedition von 1963. - Annalen des Naturhistorischen Museums in Wien 68: 653-666.

Kasy, F., 1967. Österreichische entomologische Expeditionen nach Persien und Afghanistan, Bericht über die Reise von 1965. - Annalen des Naturhistorischen Museums in Wien 70: 423-429. 
Kasy, F., 1979. Die Schmetterlingsfauna des Naturschutzgebietes Hackelsberg, Nordburgenland. - Zeitschrift der Arbeitsgemeinschaft Österreichischer Entomologen 30 Supplement: 1-27.

Kasy, F., 1983. Die Schmetterlingsfauna des WWF-Naturreservates 'Hundsheimer Berge' in Niederösterreich. Zeitschrift der Arbeitsgemeinschaft Österreichischer Entomologen 34 Supplement: 1-48.

Kemperman, T. C. M., C. Wilkinson, H. Kuroko \& T. Kumata, 1985. Japanese species of the genus Stigmella (Nepticulidae: Lepidoptera). - Insecta Matsumurana 32: 1-107.

Kimber, I., 1999-2003. UK Moths. - http://cgi.ukmoths. force9.co.uk/index.php [visited on: 26 March 2003].

Klimesch, J., 1946. Neue Stigmella-Arten (Lep., Stigmellidae). - Zeitschrift der Wiener Entomologischen Gesellschaft 31: 160-172, 1 pl.

Klimesch, J., 1951a. Nuova specie di Nepticula della Romagna (Lep., Nepticulidae). Nepticula Zangherii spec. nov. - Bollettino della Società Entomologica Italiana 81 (5-7): 61-62.

Klimesch, J., 1951b. Contributo alla fauna Lepidotterologica del Trentino. - Studi Trentini di Scienze Naturali 27 (1-3): 11-68.

Klimesch, J., 1978. Beitrag zur Kenntnis der Nepticulidenfauna von Anatolien und der Insel Rhodos (Lepidoptera, Nepticulidae). - Tijdschrift voor Entomologie 121 (5): 239-278.

Laštu̇vka, A. \& Z. Laštu̇vka, 1990. Zur Faunistik der Nepticulidae-Arten in der Tschechoslowakei (Lepidoptera). Časopis Moravskeho Muzea Vedy Prirodni 75 (1-2): 185-192.

Laštüvka, A. \& Z. Laštúvka, 1991. Weiterer Beitrag zur Erkenntnis der Nepticulidae-Arten der Tschechoslowakei (Lepidoptera). - Časopis Moravskeho Muzea Vedy Prirodni 76 (1-2): 269-275.

Laštüvka, A. \& Z. Laštủvka, 1997. Nepticulidae Mitteleuropas. Ein illustrierter Begleiter (Lepidoptera). - Konvoj, Brno. 229 pp.

Laštuivka, A. \& P. Huemer, 2002. Stigmella gutlebiella sp. n., eine neue Schmetterlingsart aus dem Iran (Lepidoptera, Nepticulidae). - Carinthia II 192/112: 603-606.

Laštüvka, Z. \& A. Laštu̇vka, 1998. Beitrag zur Kenntnis der Nepticulidenfauna Griechenlands (Lepidoptera, Nepticulidae). - Stapfia 55: 313-326.

Le Marchand, S., 1946. Stigmella prinophyllella n. sp. (Microlépidoptères, Stigmellidae). - Revue française de Lépidoptérologie 10: 280-289.

Leraut, P., 1997. Liste systématique et synonymique des Lépidoptères de France, Belgique et Corse (deuxième édition). - Supplément à Alexanor, Paris. 526 pp.

Lo Duca, R., B. Massa \& M. Concetta Rizzo, 2002. Importanza deo fragmenti di habitat naturale per le comunità di fillominatori (Insecta Diptera, Lepidoptera et Hymenoptera) e loro parassitoidi (Hymenoptera Eulophidae). Atti dell'Accademia Roveretana degli Agiati B, Classe di Scienze matematiche, fisiche e naturali (8) 2: 51-122.

Luisier, A., 1944. R. P. Cândido de Azevedo Mendes, S. J. Brotéria. Serie Ciencias Naturais 13 (1): 43-48.

Mendes, C., 1913. Lepidopteros de S. Fiel (Beira Baixa Portugal, supplemento). - Brotéria. Serie Zoológica 11 (1): $15-44$.

Mendes, C., 1918. Lepidopteros de Salamanca. - Brotéria. Serie Zoológica 16 (3): 111-129.

Menitskij, Y. L., 1984. Duby Azii [oaks of Asia]. - Nauka, Leningrad. 316 pp.
Millière, P., 1870. Catalogue raisonné des Lépidoptères du Département des Alpes-Maritimes. - Mémoires de la Société des Sciences Naturelles, des Lettres et des Beaux-Arts de Cannes et de l'arrondissement de Grasse 2: 89-219.

Morton, A., 2000. DMAP for Windows, 7.0e. - Alan Morton, Winkfield, Windsor, Berkshire.

Nieukerken, E. J. van, 1982. New and rare Nepticulidae in the Netherlands (Lepidoptera). - Entomologische Berichten (Amsterdam) 42 (7): 104-112.

Nieukerken, E. J. van, 1983. The Cistaceae-feeding Nepticulidae (Lepidoptera) of the western Palaearctic region. Systematic Entomology 8 (4): 453-478.

Nieukerken, E. J. van, 1985. A taxonomic revision of the western Palaearctic species of the subgenera Zimmermannia Hering and Ectoedemia Busck s. str. (Lepidoptera, Nepticulidae), with notes on their phylogeny. - Tijdschrift voor Entomologie 128 (1): 1-164.

Nieukerken, E. J. van, 1986a. Systematics and phylogeny of Holarctic genera of Nepticulidae (Lepidoptera, Heteroneura: Monotrysia). - Zoologische Verhandelingen 236: $1-93$.

Nieukerken, E. J. van, 1986b. A provisional phylogenetic check-list of the western Palaearctic Nepticulidae, with data on hostplants (Lepidoptera). - Entomologica Scandinavica 17 (1): 1-27.

Nieukerken, E. J. van, 1996. Nepticulidae, Opostegidae. In: O. Karsholt \& J. Razowski (eds.), The Lepidoptera of Europe. A distribitional checklist: 21-27, 300. Apollo Books, Stenstrup.

Nieukerken, E. J. van \& R. Johansson, 1987. Identity of hitherto doubtful specific names in European Nepticulidae (Lepidoptera). - Zoologische Mededelingen (Leiden) 61: 459-474.

Nieukerken, E. J. van, E. S. Nielsen, R. Johansson \& B. Gustafsson, 1990. Introduction to the Nepticulidae. - In: R. Johansson, E. S. Nielsen, E. J. van Nieukerken \& B. Gustafsson (eds.), The Nepticulidae and Opostegidae (Lepidoptera) of NW Europe. Fauna Entomologica Scandinavica 23: 11-109.

Nieukerken, E. J. v. \& Y. Liu, 2000. Nepticulidae (Lepidoptera) in China, 1. Introduction and Stigmella Schrank feeding on Fagaceae. - Tijdschrift voor Entomologie 143: 145-181.

NIMA, 2003. GEOnet Names Server. - National Imagery and Mapping Agency. http://gnswww.nima.mil/geonames/GNS/index.jsp [visited on: 21 October 2003].

Ordnance-Survey, 2003. A quarter of a million locations to find! - Ordnance Survey. http://www.ordsvy.gov.uk/ [visited on: 8 July 2003].

Patočka, J., 1980. Die Raupen und Puppen der Eichenschmetterlinge Mitteleuropas. - Monographien zur Angewandten Entomologie 23: 1-188.

Petersen, W., 1930. Die Blattminierer-Gattungen Lithocolletis und Nepticula (Lep.). Teil II: Nepticula Z. - Stettiner Entomologische Zeitung 91 (1): 1-82, 3 pls.

Peyerimhoff, H. d., 1871. [no title.]. - Mitteilungen der Schweizerischen Entomologischen Gesellschaft 3: 409415.

Popescu-Gorj, A. \& I. Draghia, 1966. Contributions à la connaissance de l'entomofaune mineuse de Roumanie. Travaux du Muséum d'Histoire Naturelle 'Grigore Antipa' 6: 99-117.

Pröse, H., 1984. Neue Ergebnisse zur Faunistik der Microlepidoptera in Bayern. 2. Beitrag. - Nachrichtenblatt der Bayerischen Entomologen 33 (4): 106-115. 
Puplesis, R. K., 1984. K sisteme molej-maljutok (Lepidoptera, Nepticulidae) palearkticeskoj fauny. - Entomologicheskoe Obozrenie 63: 582-597.

Puplesis, R. K., 1985. Contribution to the classification of the palaearctic Nepticulidae (Lepidoptera). - Entomological Review. Washington 63: 149-164.

Puplesis, R., 1994. The Nepticulidae of eastern Europe and Asia. Western, central and eastern parts. - Backhuys Publishers, Leiden. 290 pp.

Rebel, H., 1926. Beitrag zur Microlepidopterenfauna Dalmatiens. - Verhandlungen der Zoologisch-Botanischen Gesellschaft in Wien 74: (106)-(110).

Rikli, M., 1942-1943. Das Pflanzenkleid der Mittelmeerländer, 1. - Hans Huber, Bern. xvi+436 pp.

Samuelson, A., N. Evenhuis \& G. Nishida, 2001. The Insect and Spider Collections of the World. - Bishop Museum. http://www.bishopmuseum.org/bishop/ento/codens-r-us. html [visited on: 1 September 2003].

Schwarz, O., 1993. Quercus L. - In: T. G. Tutin, N. A. Burges, A. O. Chater, J. R. Edmondson, V. H. Heywood, D. M. Moore, D. H. Valentine, S. M. Walters \& D. A. Webb (eds.), Flora Europaea, 1. Psilotaceae to Platanaceae, second edition: 72-76. University Press, Cambridge.

Segerer, A. H., 1997. Verifikation älterer und fraglicher Regensburger Lepidopterenmeldungen (Insecta: Lepidoptera). - Beiträge zur bayerischen Entomofaunistik 2: $177-265$.

Shmytova, I. V., 2002. Pervoe soobshchenie o molyakh-malyutkakh (Lepidopter: Nepticulidae) Kaluzhskoj oblasti. (The first report about nepticulids (Lepidoptera: Nepticulidae) of the Kaluga Area). - Russian Entomological Journal 11 (3): 311-312.

Skala, H., 1937. Minen aus Mittel- und Südeuropa. Zeitschrift des Österreichischen Entomologen-Vereins 22: 10-11, 19-20.

Skala, H., 1938. Einiges über Falter-Minen aus dem Mediterrangebiet. - Zeitschrift des Österreichischen Entomologen-Vereins 23: 8-10, 30-31, 43-46.

Sorhagen, L., 1922. Beiträge zur Biologie Europäischer Nepticula-Arten. - Archiv fur Naturgeschichte 88A: 9-60.

Southwood, T. R. E., 1961. The number of species of insect associated with various trees. - Journal of Animal Ecology 30: 1-8.

Stainton, H. T., 1869. Tineina observed at Cannes and Mentone in February and March 1867. - In: H. T. Stain- ton (ed.), The Tineina of Southern Europe. 216-232. John van Voorst, London.

Steuer, H., 1998. Neue Daten zur Schmetterlingsfauna (Insecta, Lepidoptera) der Umgebung von Bad Blankenburg (Thuringen). - Rudolstädter Naturhistorische Schriften 9: 29-34.

Sulcs, A. \& I. Sulcs, 1989. Neue und wenig bekannte Arten der Lepidopteren-Fauna Lettlands. 12. Mitteilung. - Notulae Entomologicae 69: 67-72.

Szőcs, J., 1968. Some unknown data concerning miners (Lepidoptera). - Acta Zoologica Academiae Scientiarum Hungariae 14: 225-231.

Szőcs, J., 1977. A lepkehernyók természetes tápnövényei, 3. Die natürlichen Futterpflanzen der Schmetterlingsraupen, 3. - Folia Entomologica Hungarica, s.n. 30 (2): 143-150.

Times, 2000. The Times comprehensive atlas of the world, 10th edition 911. - Times Books, London. 544 pp.

Tokár, Z., I. Richter, G. Pastorális \& F. Slamka, 2002. New and interesting records of Lepidoptera of Slovakia from the years 1998-2001. - Entomofauna Carpathica 14: 1-11.

Vári, L., 1950. Nederlandse Lepidoptera. 5e Faunistische Mededeling. - Entomologische Berichten, Amsterdam 13 (306): 180-184.

Vives Moreno, A., 1991. Catalogo sistematico y sinonimico de los Lepidopteros de la Peninsula Iberica y Baleares (Insecta: Lepidoptera). - Secretaría General Técnica, Ministerio de Agricultura, Pesca y Alimentación, Madrid. 378 pp.

Zangheri, S., 1969. Regno Animale. Sottord. Heterocera. In: S. Zangheri (ed.), Repertorio sistematico e topografico della Flora e Fauna vivente e fossile della Romagna, 3. Memorie fuori Serie 3: 855-1015. Museo Civico di Storia Naturale, Verona.

Zeller, P. C., 1839. Versuch einer naturgemässen Eintheilung der Schaben. - Isis, Jena 1838: 167-220.

Zeller, P. C., 1848. Die Gattungen der mit Augendeckeln versehenen blattminirenden Schaben. - Linnaea Entomologica 3: 248-344.

Zerkowitz, A., 1946. The Lepidoptera of Portugal. - Journal of the New York Entomological Society 54: 57-87, $115-165,211-261$.

Received: 9 September 2003

Accepted: 17 October 2003

Figs. 90-98. Stigmella spp., male genitalia, photographs, slides by EvN, unless otherwise mentioned. - 90, S. fasciata, slide 3462; 91, S. suberivora; 92, 93, S. ilicifoliella, slides 3340 and 3443; 94, 95, S. cocciferae, slide 2986; 96, S. kasyi, holotype, slide RJ1528; 97, 98, S. basiguttella, slide 2555. Scale lines $100 \mu \mathrm{m}$. 

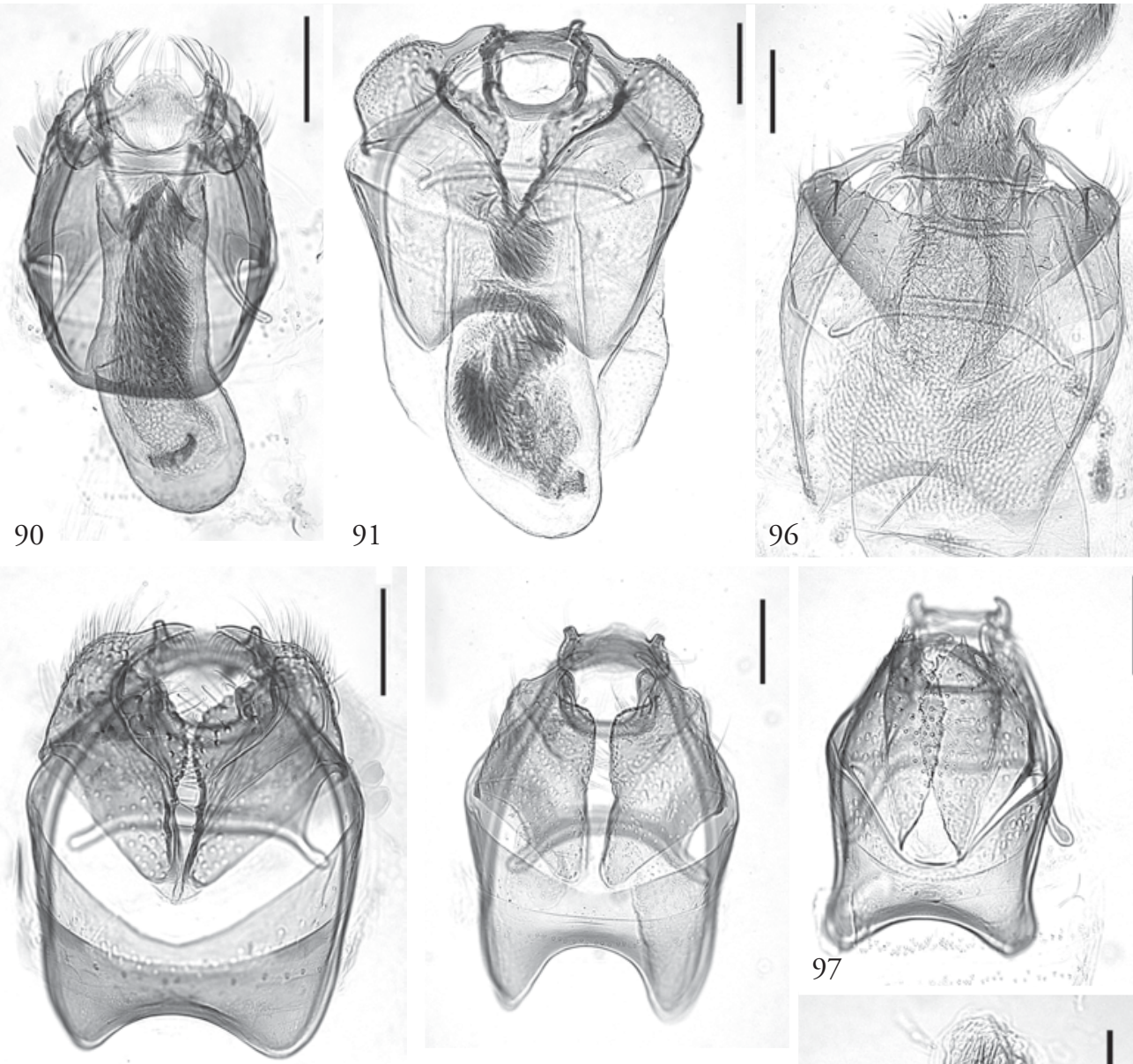

92

94
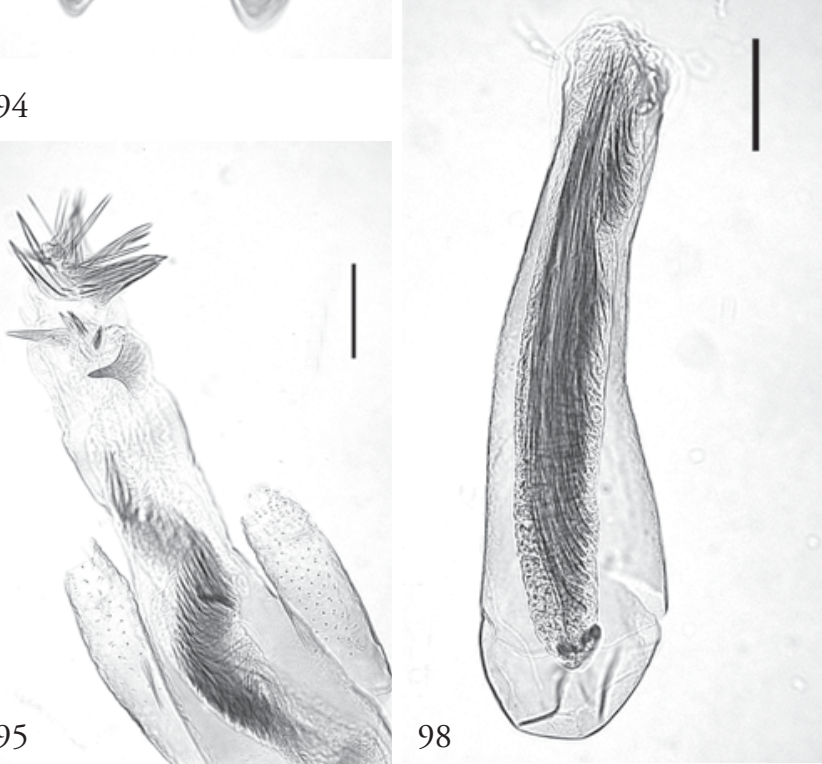

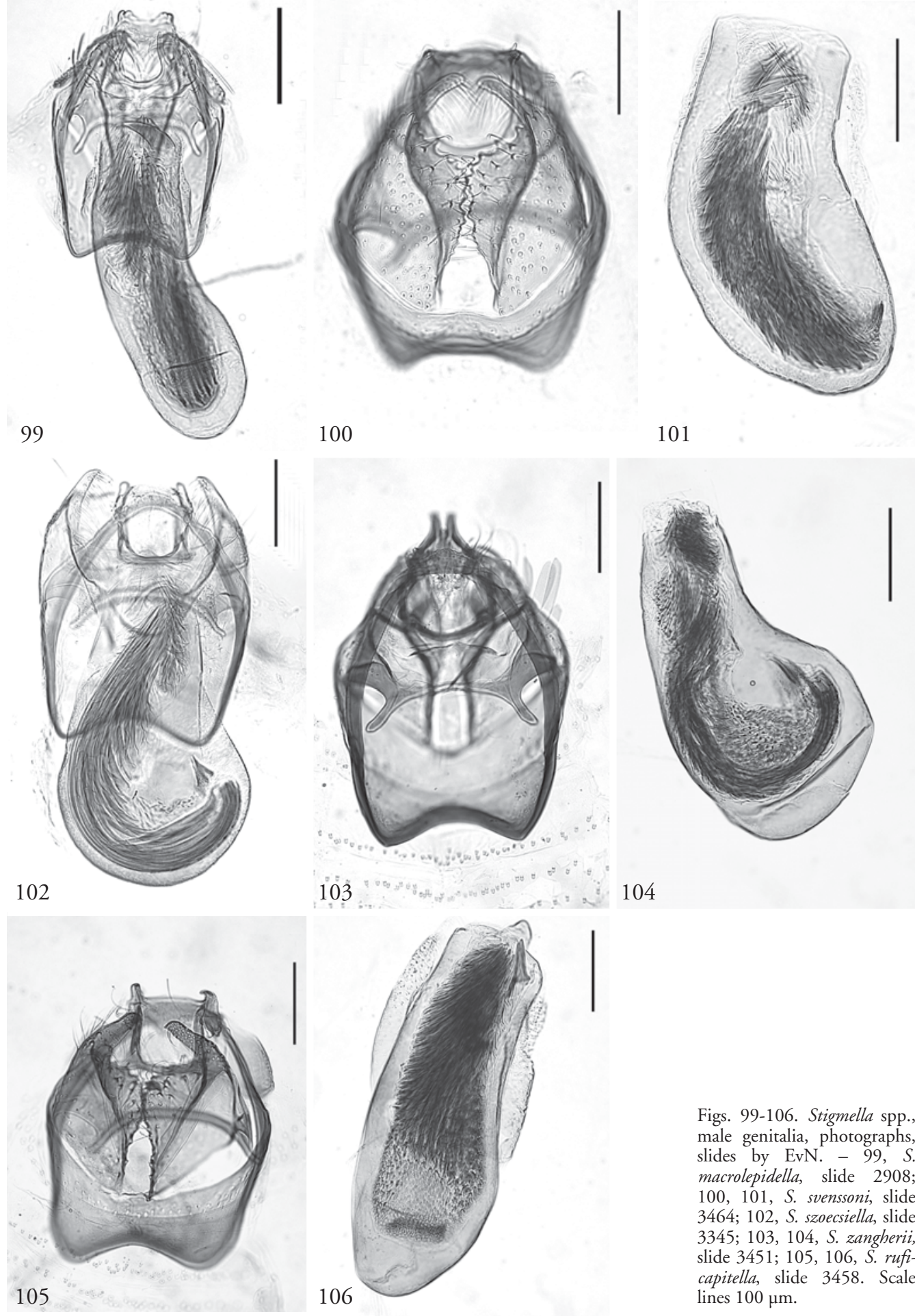

Figs. 99-106. Stigmella spp., male genitalia, photographs, slides by EvN. - 99, S. macrolepidella, slide 2908; 100, 101, S. svenssoni, slide 3464; 102, S. szoecsiella, slide $3345 ; 103,104$, S. zangherii, slide $3451 ; 105,106, S$. ruficapitella, slide 3458. Scale lines $100 \mu \mathrm{m}$. 


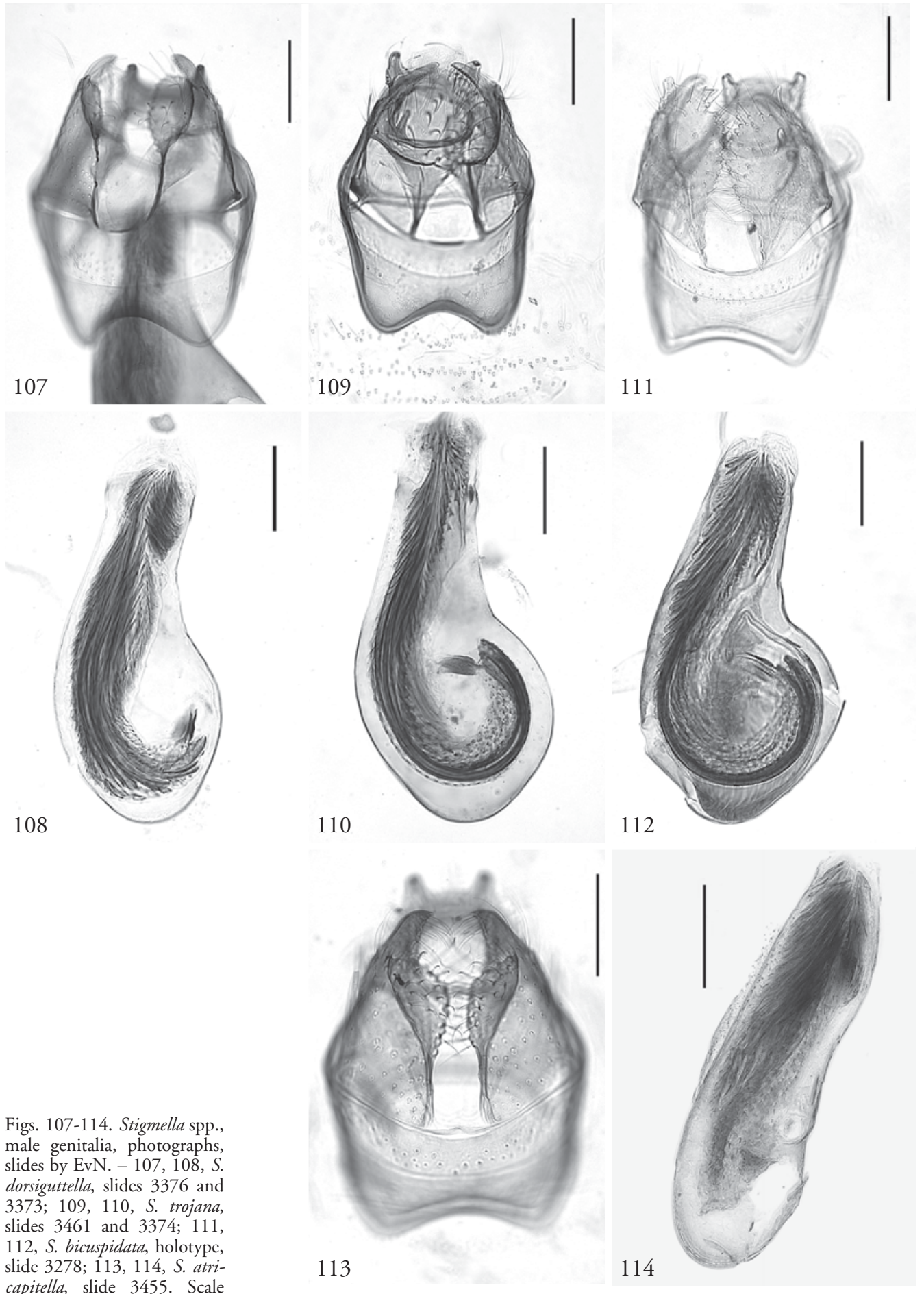

capitella, slide 3455. Scale lines $100 \mu \mathrm{m}$. 

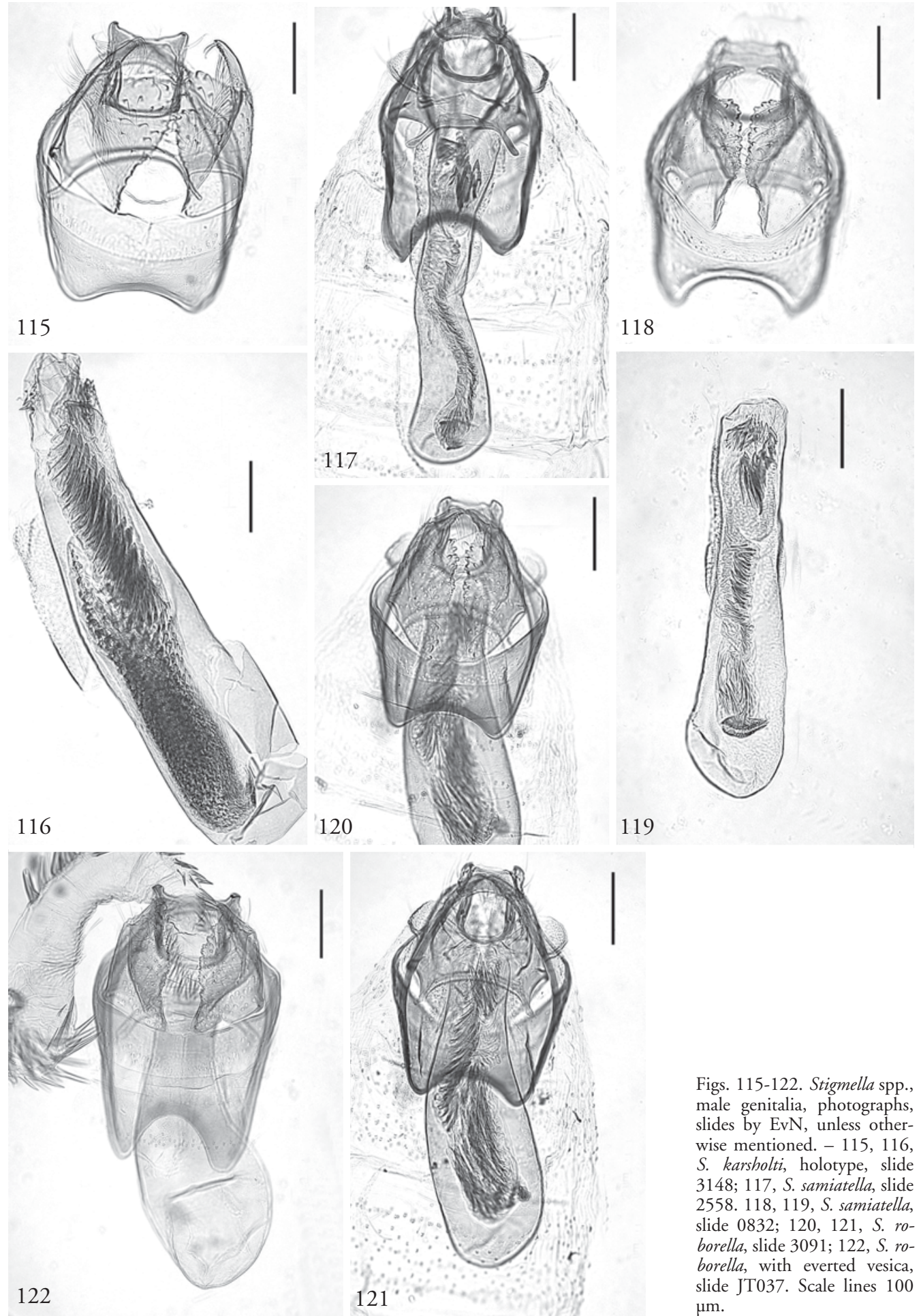

Figs. 115-122. Stigmella spp., male genitalia, photographs, slides by EvN, unless otherwise mentioned. - 115, 116, S. karsholti, holotype, slide 3148; 117, S. samiatella, slide 2558. 118, 119, S. samiatella, slide $0832 ; 120,121$, S. roborella, slide 3091; 122, S. roborella, with everted vesica, slide JT037. Scale lines 100 $\mu \mathrm{m}$. 

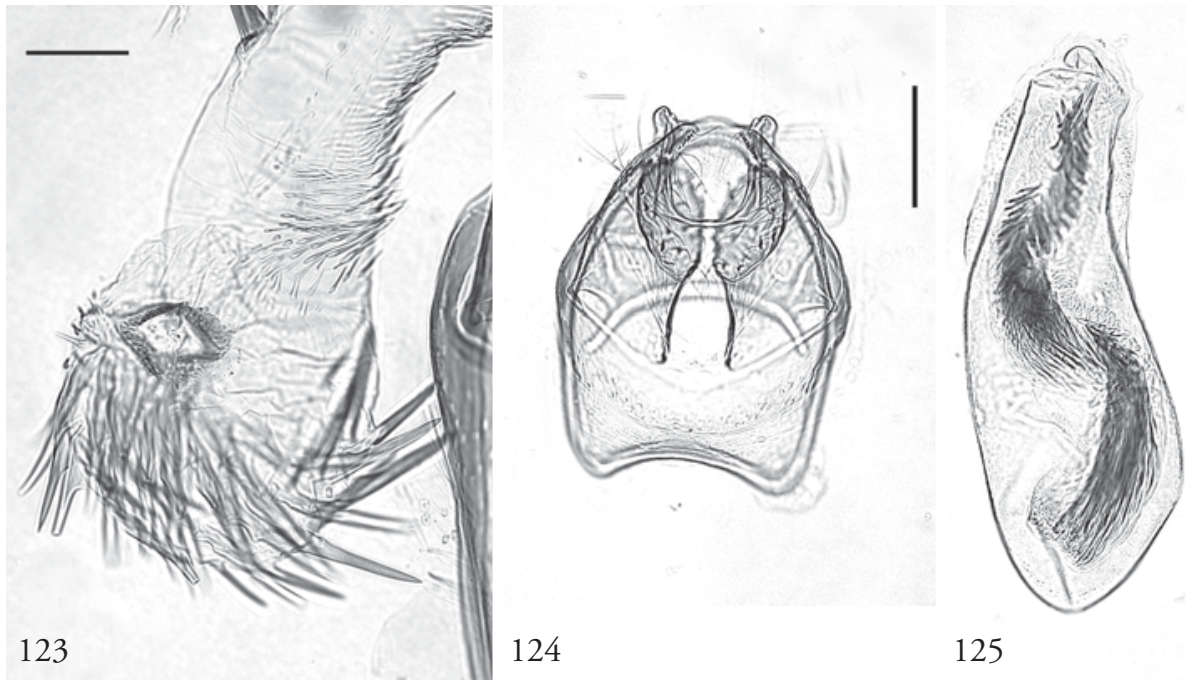

124

125
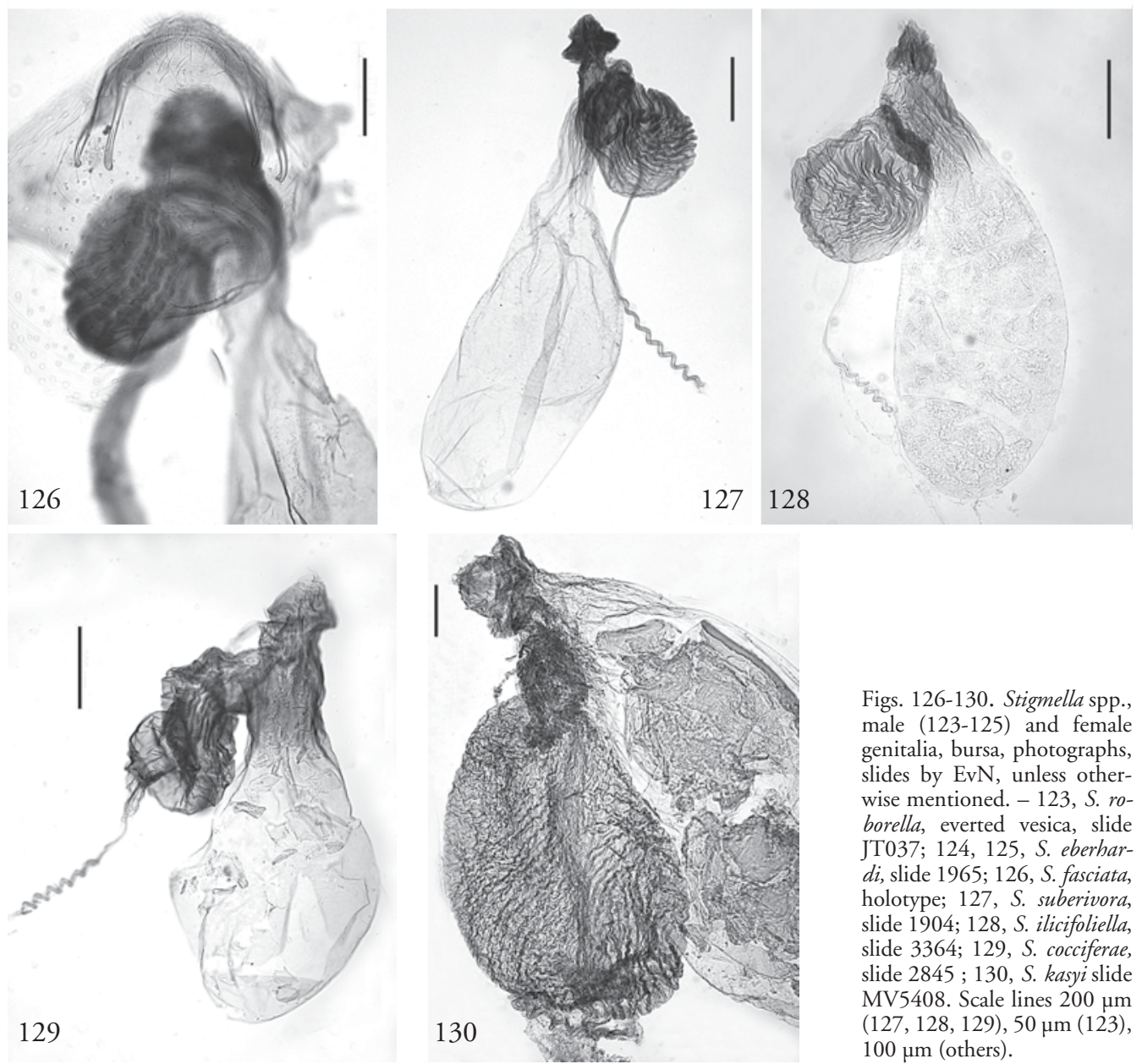

Figs. 126-130. Stigmella spp., male (123-125) and female genitalia, bursa, photographs, slides by EvN, unless otherwise mentioned. - 123, S. roborella, everted vesica, slide JT037; 124, 125, S. eberhardi, slide 1965; 126, S. fasciata, holotype; 127 , S. suberivora, slide 1904; 128, S. ilicifoliella, slide 3364; 129, S. cocciferae, slide $2845 ; 130$, S. kasyi slide MV5408. Scale lines $200 \mu \mathrm{m}$ (127, 128, 129), $50 \mu \mathrm{m}$ (123), $100 \mu \mathrm{m}$ (others). 

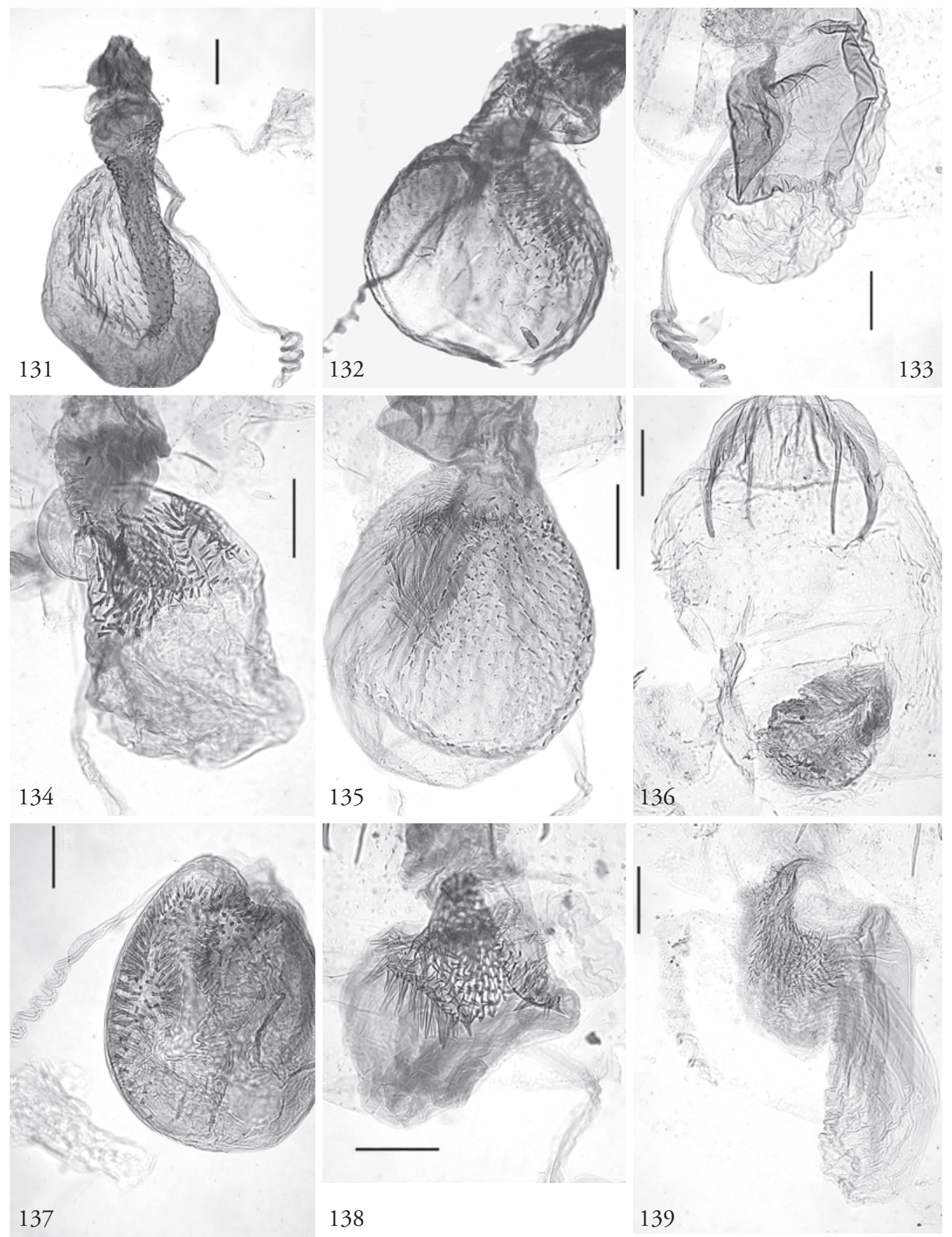

Figs. 131-139. Stigmella spp., female genitalia, bursa, photographs, slides by EvN, unless otherwise mentioned. - 131, $S$. basiguttella, slide 2562; 132, S. macrolepidella, slide 2904; 133, S. svenssoni, slide 3459; 134, S. szoecsiella, slide 3345; 135 , S. zangherii, slide 3348; 136, 137, S. dorsiguttella, slide 3475, accessory sac broken posteriorly; 138, S. trojana, slide 3375; 139, S. ruficapitella, slide 3467; Scale lines $100 \mu \mathrm{m}$. 


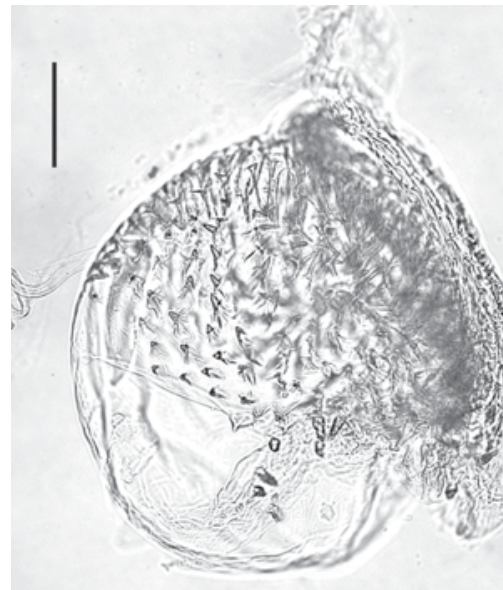

141
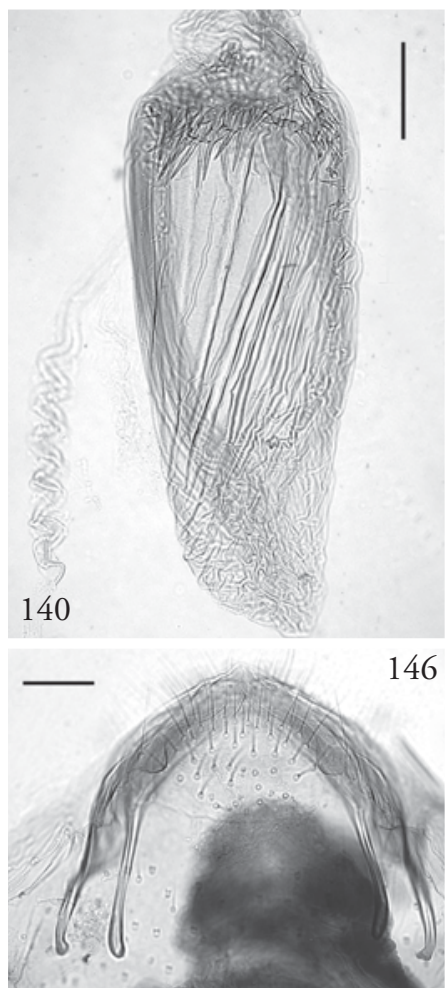
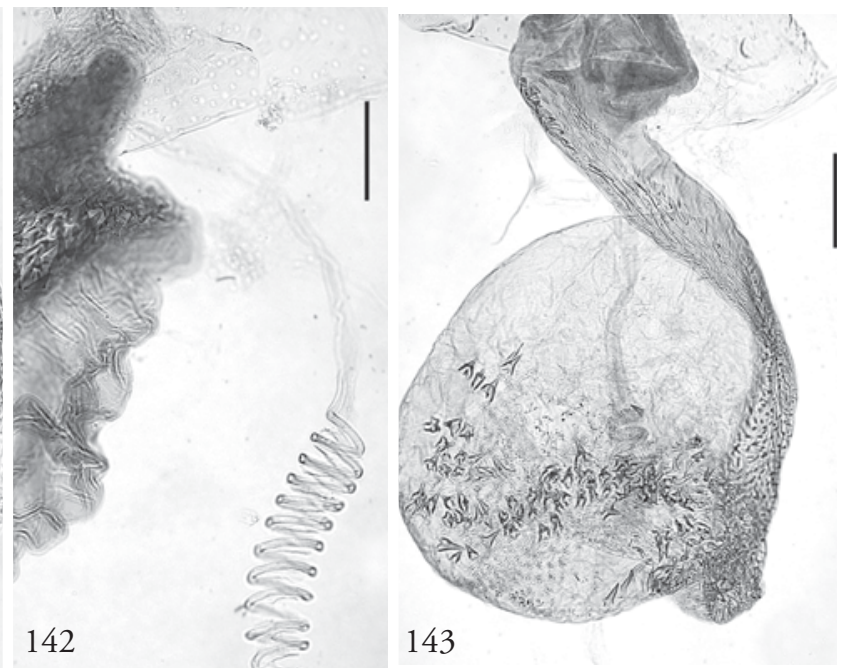

144
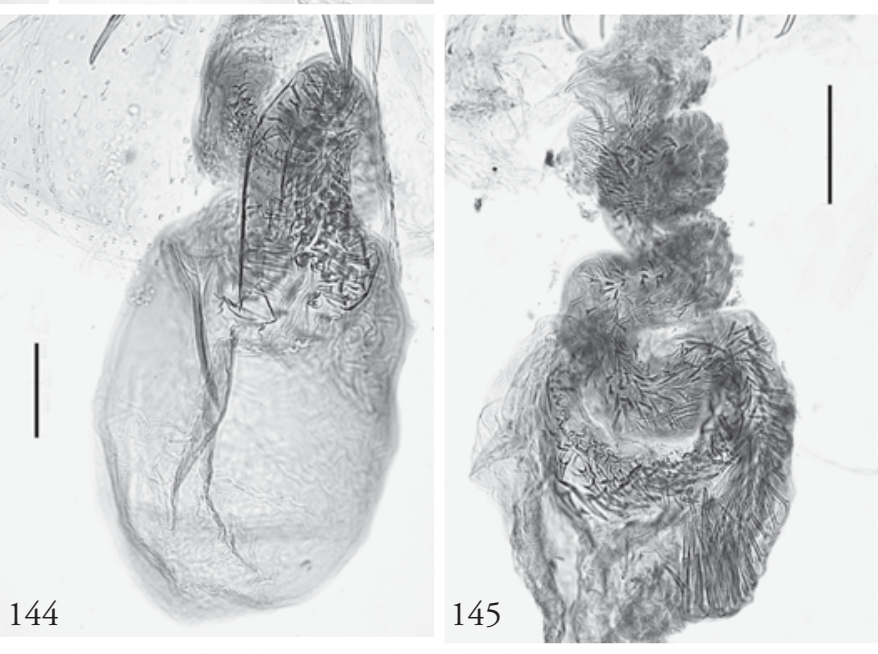

148
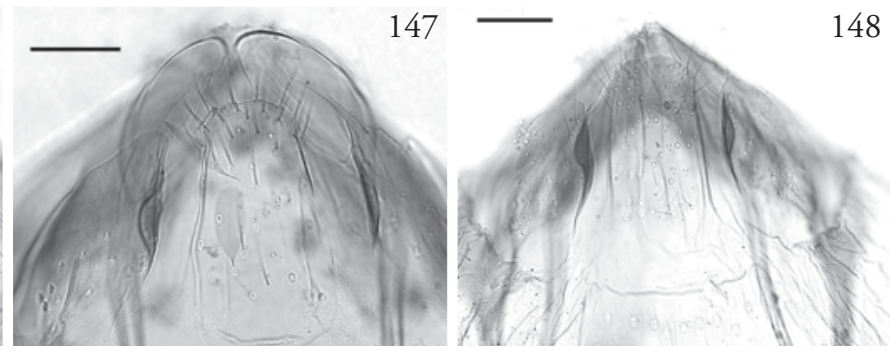

Figs. 140-148.Stigmella spp., female genitalia, bursa (140-145), abdominal tip with tergite viii (146-148), photographs, slides by EvN, unless otherwise mentioned. - 140, S. atricapitella, slide 3466; 141, 142, S. karsholti, slides 3164 and 3160; 143 , S. samiatella, slide 3327; 144, S. roborella, slide 2726; 145, S. eberhardi, slide 3366. - 146, S. fasciata, holotype; 147, S. suberivo$\mathrm{ra}$, slide 3456; 148, S. ilicifoliella, slide 2517. Scale lines $100 \mu \mathrm{m}$ (140-145), $50 \mu \mathrm{m}$ (146-148). 

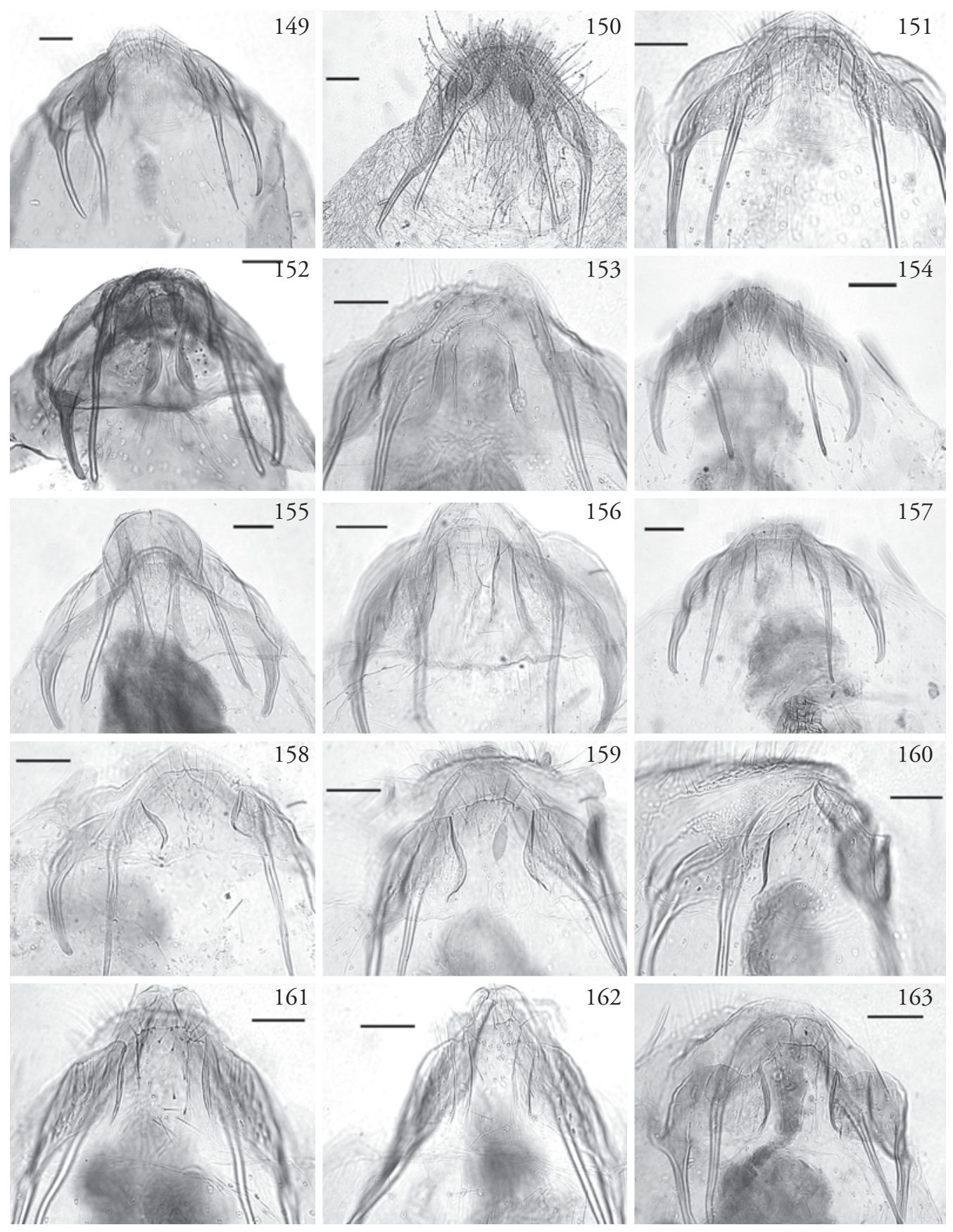

Figs. 149-163. Stigmella spp., female genitalia, abdominal tip with tergite 8, photographs, slides by EvN. - 149, S. cocciferae, slide 2845; 150, S. kasyi slide MV5408; 151, S. basiguttella, slide 2562; 152, S. macrolepidella, slide 2904; 153, S. svenssoni, slide 3459; 154, S. szoecsiella, slide 3345; 155, S. zangherii, slide 3348; 156, S. dorsiguttella, slide 3475; 157, S. trojana, slide 3375; 158, S. ruficapitella, slide 3467; 159, S. atricapitella, slide 3460; 160, S. karsholti, slide 3160; 161, S. samiatella, slide 2725; 162, S. roborella, slide 2726; 163, S. eberhardi, , slide 3366. Scale lines $50 \mu \mathrm{m}$. 

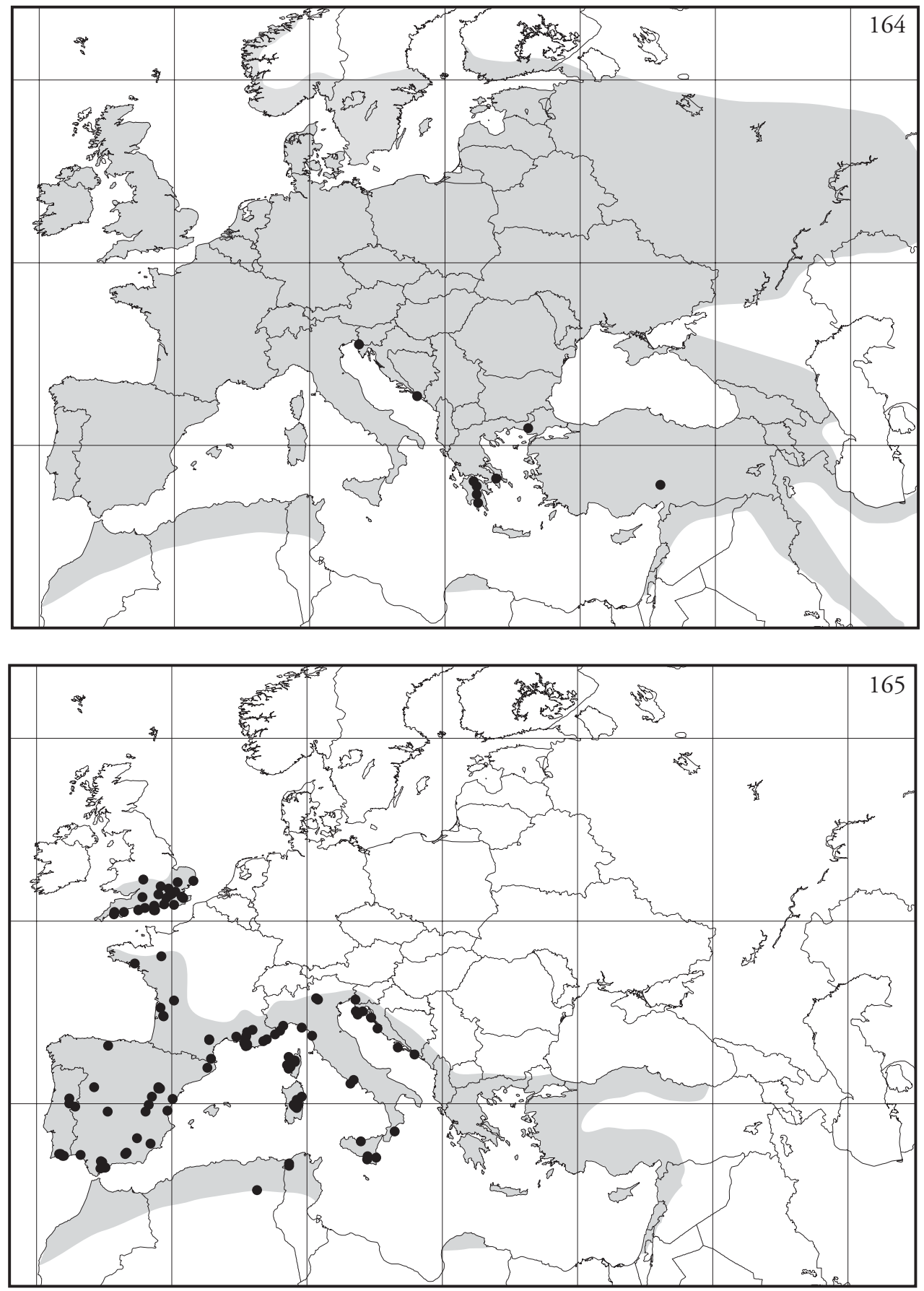

Figs. 164-179 (pages 363-370). Distribution maps of Stigmella spp. - 164, S. fasciata; shaded area represents approximate natural distribution area of Quercus; 165, S. suberivora, shaded area represents combined distribution of evergreen oaks (Quercus coccifera, ilex, rotundifolia and suber), in Britain area of planted Q. ilex. 

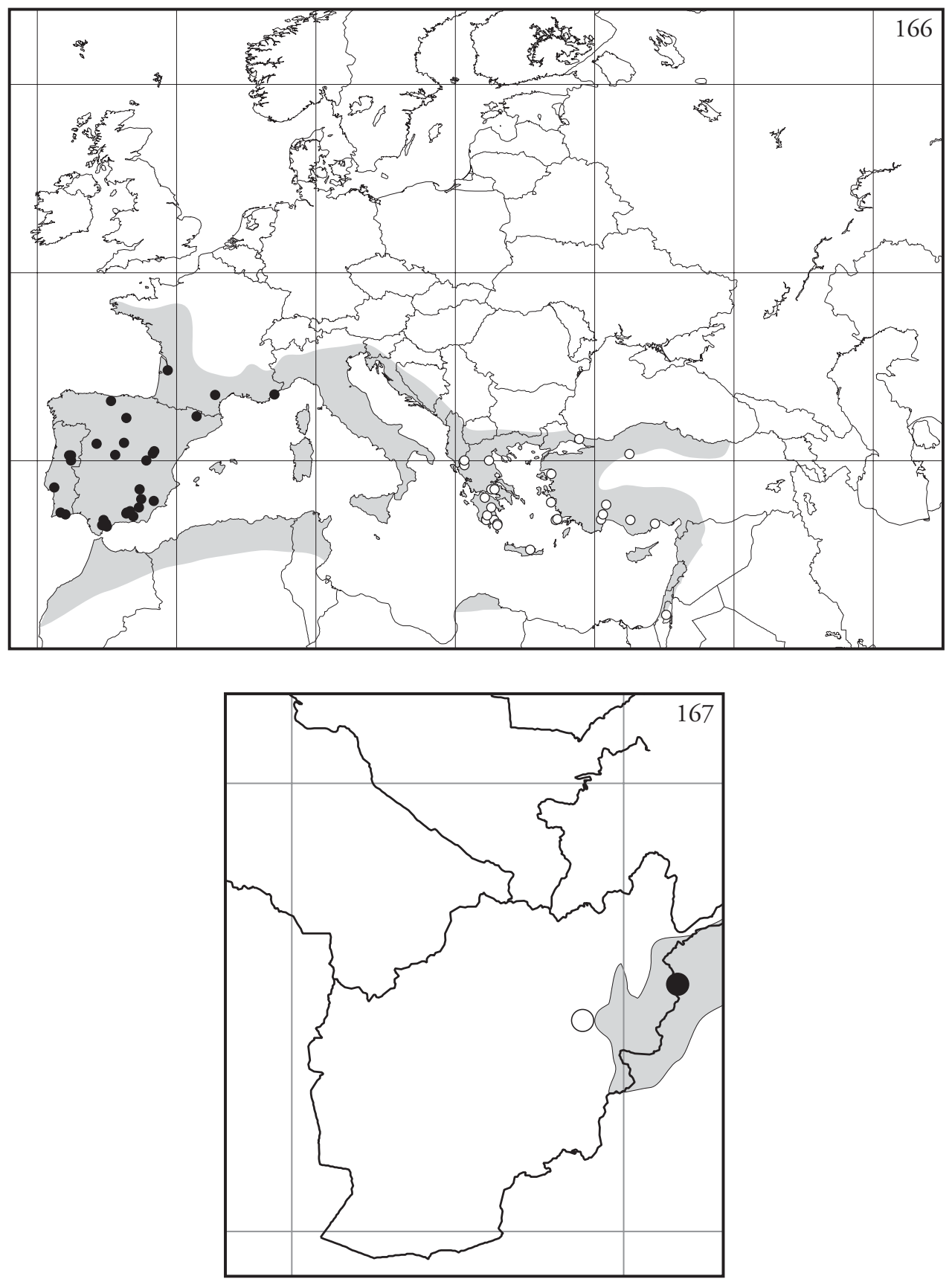

166, S. ilicifoliella (black dots) and S. cocciferae (open dots); shaded area represents combined natural distribution of evergreen oaks (Quercus coccifera, ilex, rotundifolia and suber); 167, S. kasyi, the open dot represents the female; shaded area represents approximate combined distribution area of Quercus species: Q. baloot, Q. dilatata and Q. semecarpifolia. 

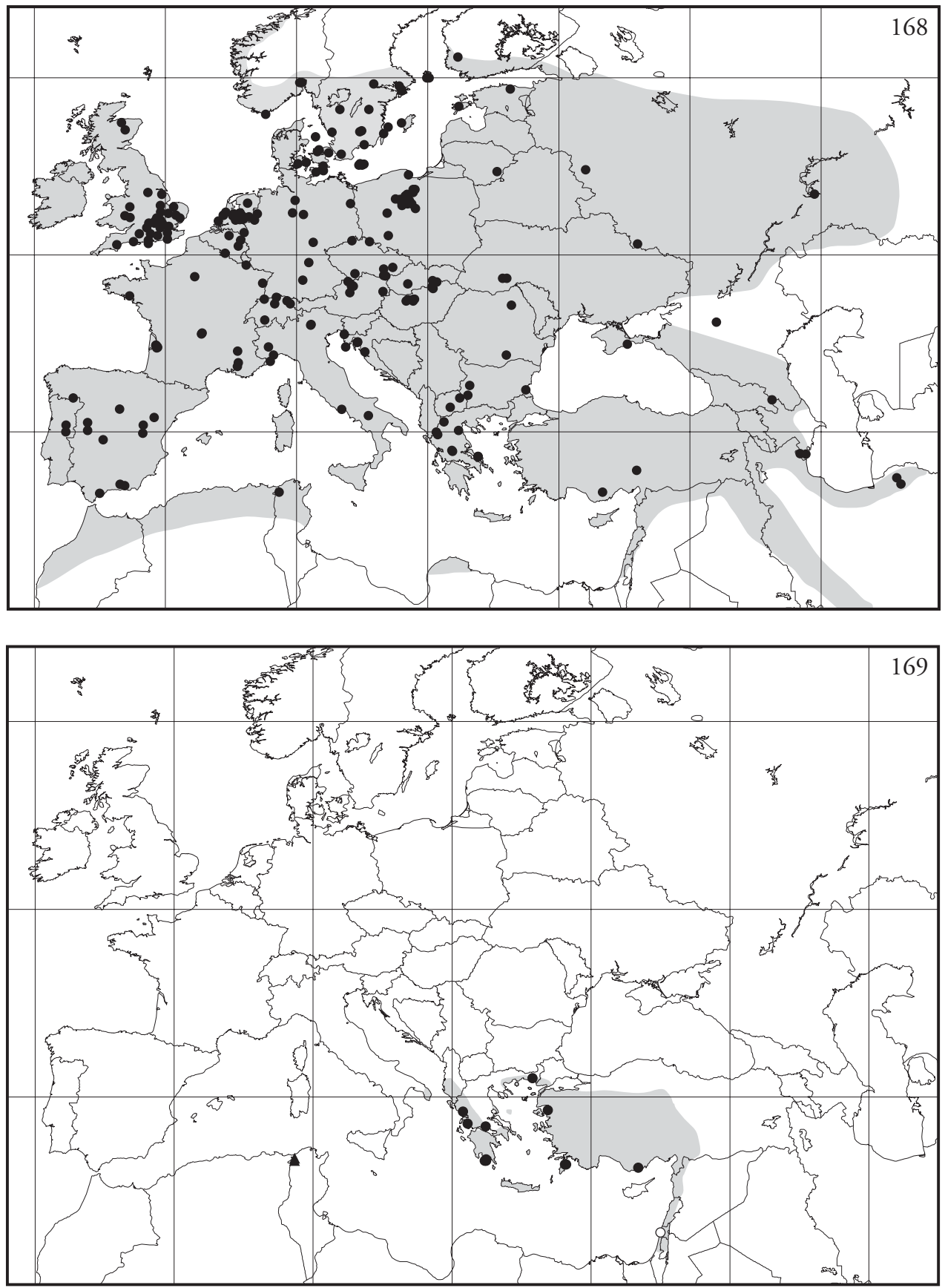

168, S. basiguttella, shaded area represents approximate natural distribution area of Quercus, dot outside distribution area of Quercus north of Caucasus refers to mine records from Kalmykia on planted oaks; 169, S. macrolepidella (dots) and S. karsholti (triangles, Tunisia); the open dot in Israel refers to the record of leafmines on Quercus ithaburensis ssp. ithaburensis which belong possibly to $S$. macrolepidella; shaded area represents approximate distribution area of Quercus ithaburensis, host of $S$. macrolepidella. 

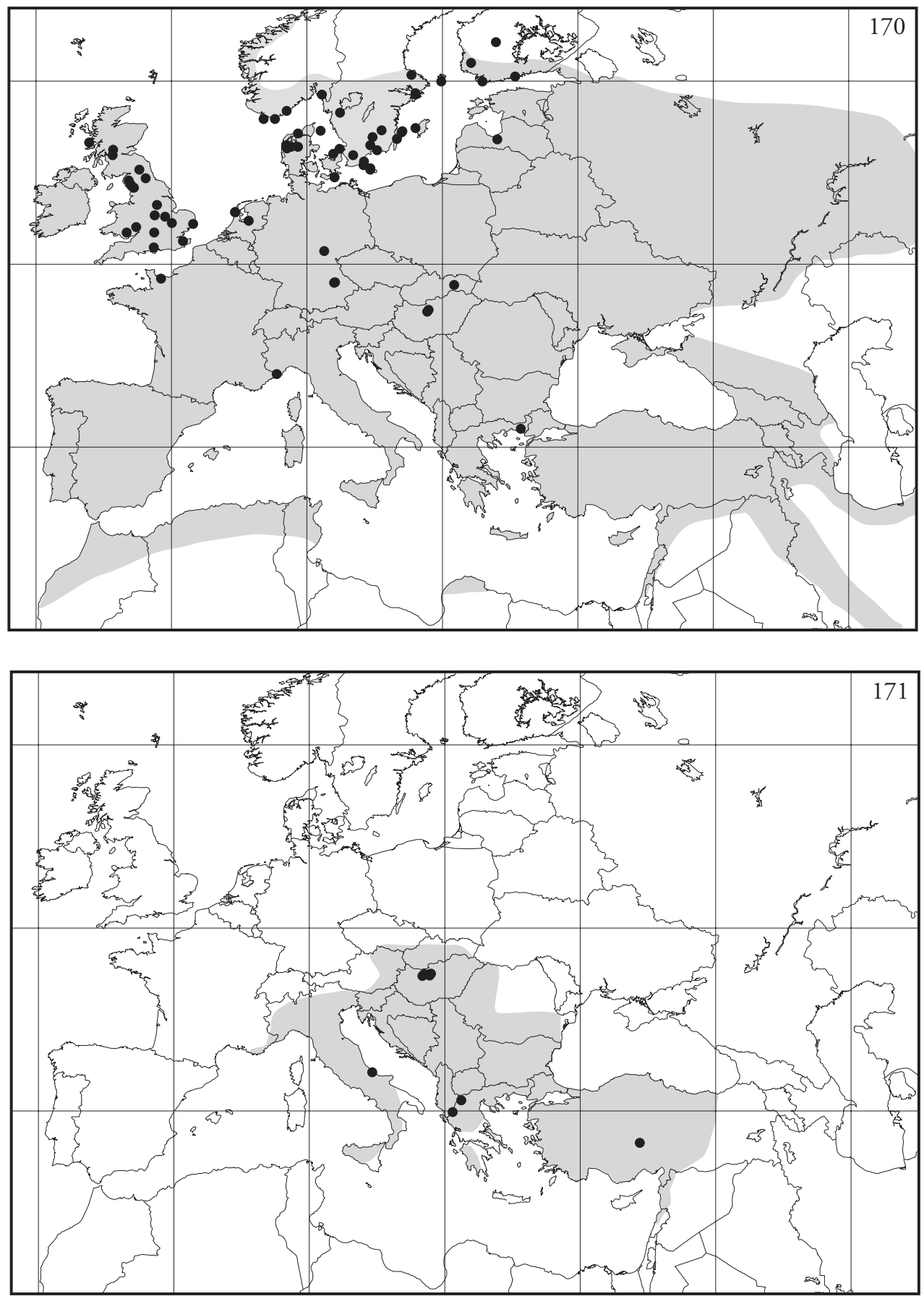

170, S. svenssoni; shaded area represents approximate natural distribution area of Quercus, 171, S. szoecsiella; shaded area represents approximate distribution area of Quercus cerris, the most common host. 

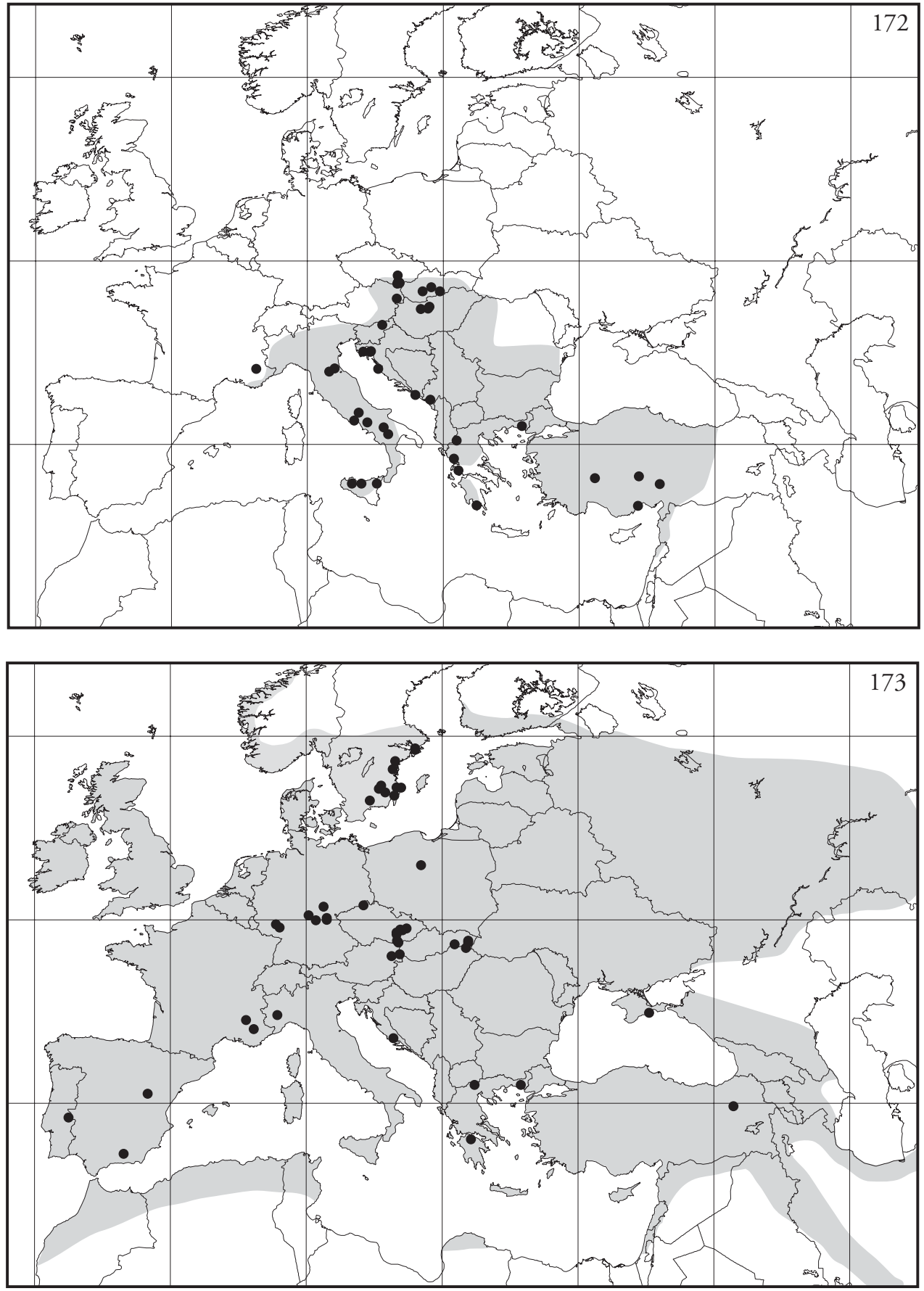

172, S. zangherii; shaded area represents approximate distribution area of Quercus cerris, the most common host; 173, S. dorsiguttella; shaded area represents approximate natural distribution area of Quercus. 

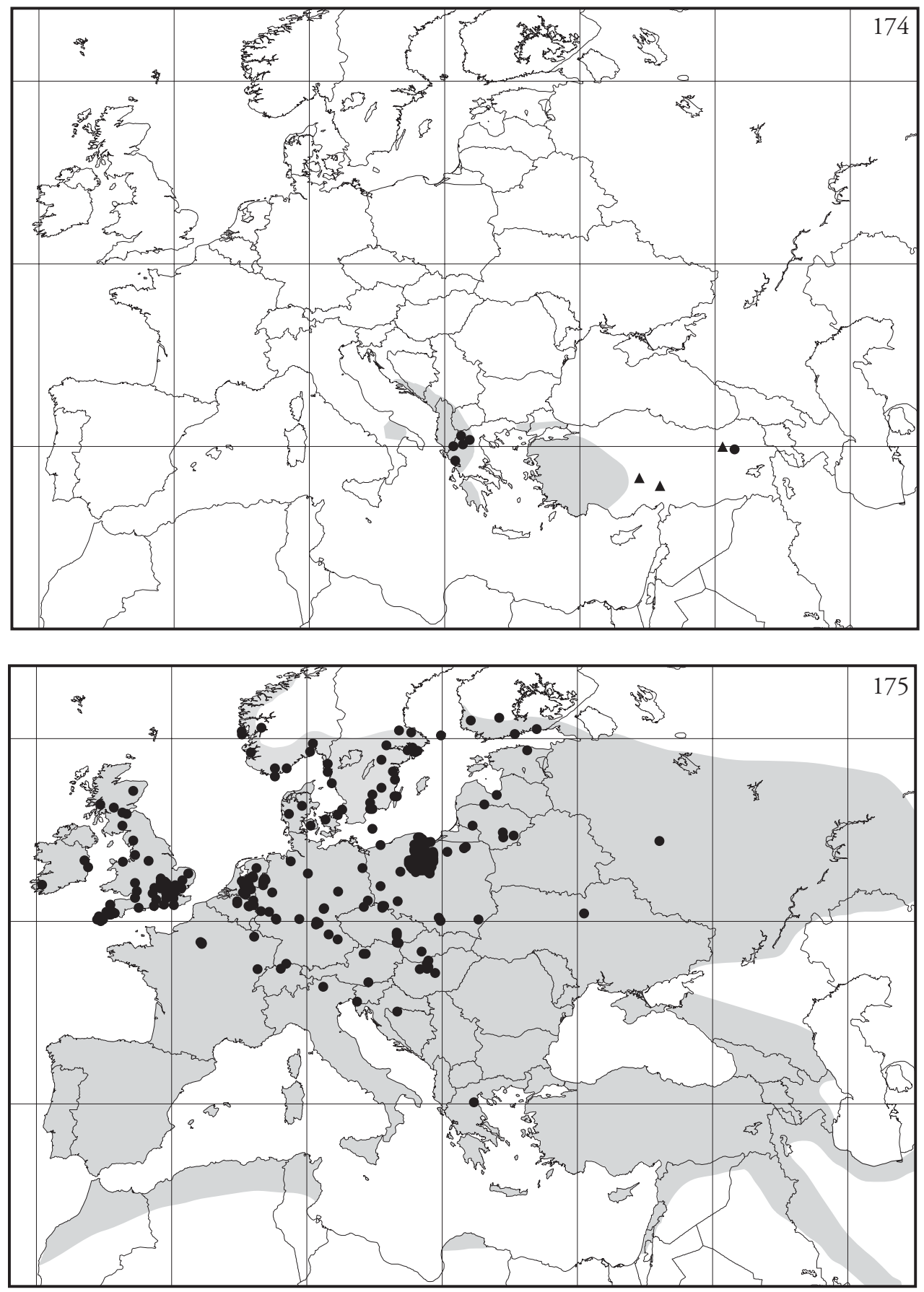

174, S. trojana (dots) and S. bicuspidata (triangles); shaded area represents approximate distribution area of Quercus trojana, host of S. trojana; 175, S. ruficapitella; shaded area represents approximate natural distribution area of Quercus; 

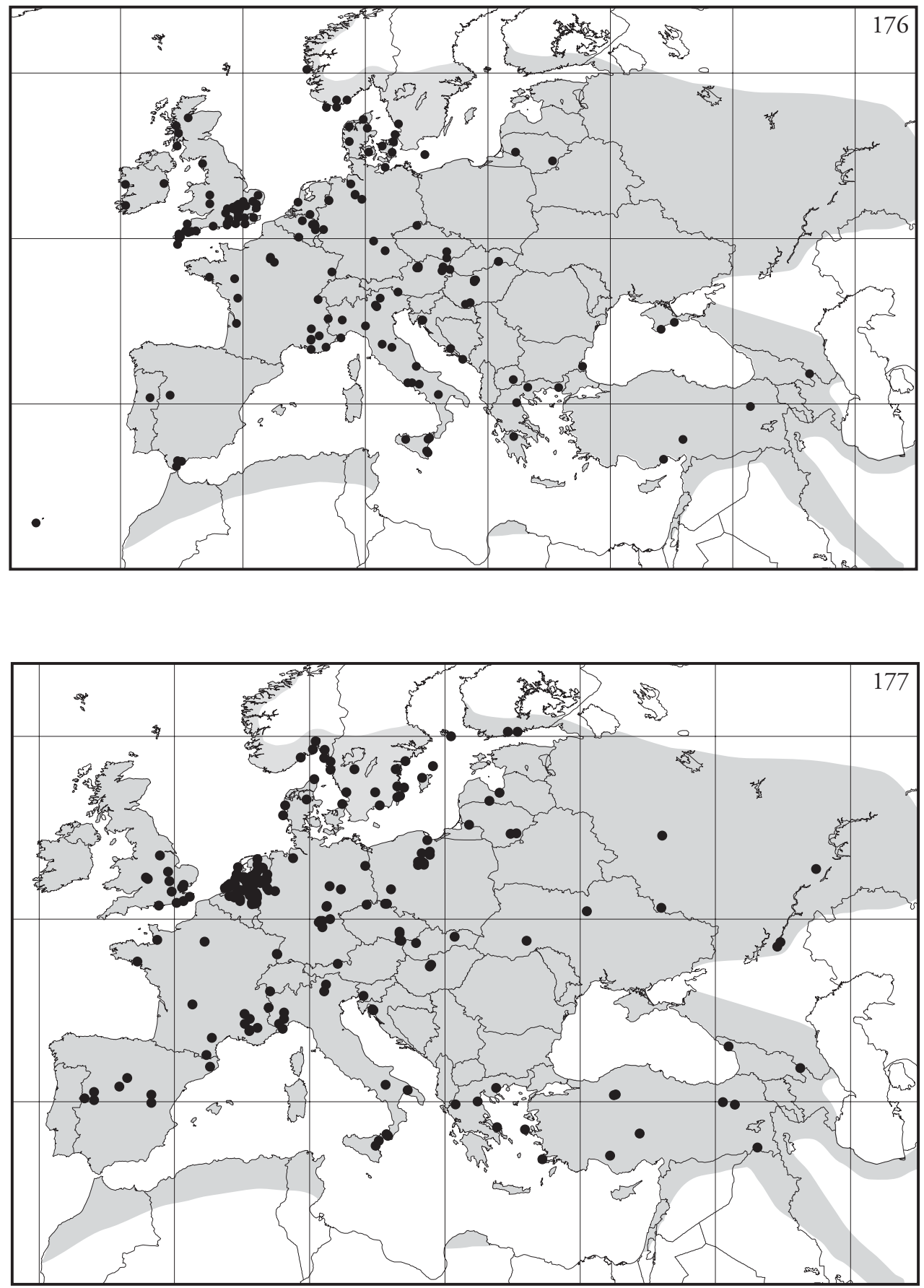

176, S. atricapitella; 177, S. samiatella; shaded areas represents approximate natural distribution area of Quercus; 

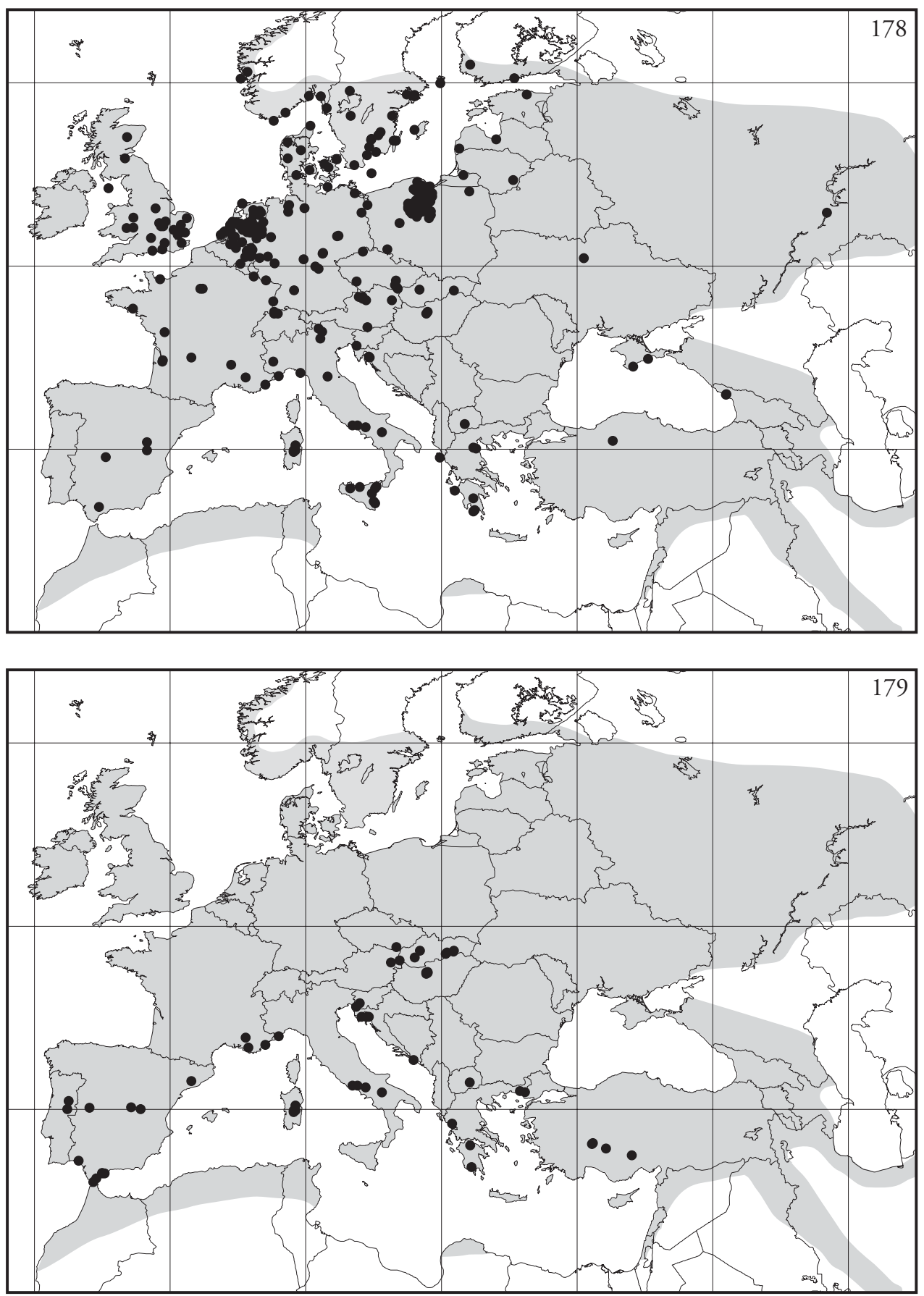

178, S. roborella; 179, S. eberhardi; shaded areas represents approximate natural distribution area of Quercus. 